\title{
LAGRANGIAN-INVARIANT SHEAVES AND FUNCTORS FOR ABELIAN VARIETIES
}

\author{
ALEXANDER POLISHCHUK
}

\begin{abstract}
We partially generalize the theory of semihomogeneous bundles on an abelian variety $A$ developed by Mukai [14]. This involves considering abelian subvarieties $Y \subset$ $X_{A}=A \times \hat{A}$ and studying coherent sheaves on $A$ invariant under the action of $Y$. The natural condition to impose on $Y$ is that of being Lagrangian with respect to a certain skew-symmetric biextension $\mathcal{E}$ of $X_{A} \times X_{A}$ by $\mathbb{G}_{m}$. We prove that in this case any $Y$-invariant sheaf is a direct sum of several copies of a single coherent sheaf. We call such sheaves Lagrangian-invariant (or LI-sheaves). We also study LI-functors $D^{b}(A) \rightarrow D^{b}(B)$ associated with kernels in $D^{b}(A \times B)$ that are invariant with respect to some Lagrangian subvariety in $X_{A} \times X_{B}$. We calculate their composition and prove that in characteristic zero it can be decomposed into a direct sum of LI-functors. In the case $B=A$ this leads to an interesting central extension of the group of symplectic automorphisms of $X_{A}$ in the category of abelian varieties up to isogeny.
\end{abstract}

\section{INTRODUCTION}

Derived categories of coherent sheaves on smooth projective varieties have been playing an increasingly significant role in modern algebraic geometry. They serve as a motivation and a testing ground for constructions in noncommutative geometry of smooth proper dg-categories (see [10]) and have interesting links to representation theory, mirror symmetry and other areas (see [2] and references therein). One of the first results that revealed the importance of derived categories was the equivalence of the derived categories $D^{b}(A) \simeq D^{b}(\hat{A})$ for an abelian variety $A$ and its dual $\hat{A}$. established by Mukai [13]. The present paper continues the line of study of [18], where it was shown that the group of autoequivalences of $D^{b}(A)$ is closely related to the group $U(A \times \hat{A})$ of "symplectic" automorphisms of $A \times \hat{A}$ (the term "symplectic" is explained below). Namely, we study endofunctors of $D^{b}(A)$ related to symplectic automorphisms of $A \times \hat{A}$ in the bigger category $\mathcal{A} b_{\mathbb{Q}}$ of abelian varieties up to isogeny. More generally, we study a natural class of LI-functors between derived categories of abelian varieties governed by Lagrangian correspondences between the corresponding varieties $A \times \hat{A}$. Our motivation is twofold. On the one hand, such functors could be useful for the Manin's real multiplication program (see [11]) if one tries to approach it using the categories of holomorphic bundles on noncommutative tori described in [24]. On the other hand, as will be shown in a sequel to this paper, using these functors one can realize the Bridgeland stability space of an abelian surface $A$ as a homogeneous space for certain covering of the group $U(A \times \hat{A}, \mathbb{R})$ of $\mathbb{R}$-points of the algebraic group of symplectic automorphisms of $A \times \hat{A}$ (more precisely, one should consider the connected component of the stability space described in [3]).

Supported in part by the NSF grant DMS-1001364. 
As in [20], with every abelian variety $A$ we associate a "symplectic" object: the abelian variety $X_{A}=A \times \hat{A}$ together with a skew-symmetric biextension $\mathcal{E}=p_{14}^{*} \mathcal{P} \otimes p_{23}^{*} \mathcal{P}^{-1}$ of $X_{A} \times X_{A}$ (where $\mathcal{P}$ is the Poincaré line bundle on $A \times \hat{A}$ ). There is an analog of the Schrödinger representation of the Heisenberg group in this setting obtained by considering the action of a certain Heisenberg extension $\mathbf{H}$ of $X_{A}$ on $D^{b}(A)$ by translations and tensoring with line bundles in $\operatorname{Pic}^{0}(A)$ (see Sec. 2.1). One can show that under some technical assumptions, the stabilizer of an endosimple object of $D^{b}(A)$ is always an isotropic subgroup $Y \subset X_{A}$ with respect to the biextension $\mathcal{E}$ (see Remark 2.3.3). We study the case when this subgroup is a Lagrangian, i.e., when we have a duality between $Y$ and $X_{A} / Y$ (we show that this is equivalent to $Y$ having the maximal possible dimension $\left.\operatorname{dim} A=\operatorname{dim} X_{A} / 2\right)$. Our first main result is the description of the subcategory of invariants in $D^{b}(A)$ of a Lagrangian abelian subvariety $Y \subset X_{A}$ (see Theorem 2.4.5). More precisely, to define this subcategory of invariants we need to choose a line bundle $\alpha$ on $Y$ that gives a lifting of $Y$ to the Heisenberg groupoid $\mathbf{H}$ of $A$ (see Sec. $2.2)$. Then we can define the subcategory of $(Y, \alpha)$-invariant objects in $D^{b}(A)$. We show that this subcategory is generated by a single endosimple coherent sheaf $S_{Y, \alpha}$ and that every $(Y, \alpha)$-invariant coherent sheaf is a direct sum of several copies of $S_{Y, \alpha}$. We call such sheaves Lagrangian-invariant (LI-sheaves for short). Note that the case when the projection $Y \rightarrow A$ is an isogeny corresponds to semihomogeneous vector bundles on $A$ (see [14]). However, our condition of $(Y, \alpha)$-invariance is stronger than the condition of invariance under all functors corresponding to points in $Y(k)$. Namely, the condition for a sheaf $F \in \operatorname{Coh}(A)$ to be $(Y, \alpha)$-invariant is given by a certain isomorphism over $A \times Y$ (see Sec. 2.4). This accounts for the fact that nontrivial extensions of $S_{Y, \alpha}$ with itself are not $(Y, \alpha)$-invariant (see Example 2.4.1).

Note that finite Heisenberg groups appear in our picture as well. Namely, for a pair of Lagrangian subvarieties $(Y, Z)$ in $X_{A}$ equipped with liftings to the Heisenberg groupoid, we have a natural group extension $G$ of the intersection $Y \cap Z$ by $\mathbb{G}_{m}$, which is a Heisenberg group in the case when $Y \cap Z$ is finite (this construction goes back to [20]). Generalizing Mumford's theory of theta groups we show that the space $\operatorname{Hom}_{D^{b}(A)}^{*}\left(S_{Y, \alpha}, S_{Z, \beta}\right)$ is an irreducible representation of the Heisenberg group $G$ (see Corollary 3.2.12).

For a pair of abelian varieties $A$ and $B$ we consider Lagrangian correspondences from $X_{A}$ to $X_{B}$, i.e., Lagrangian abelian subvarieties $L \subset X_{A} \times X_{B}$ with respect to the difference of biextensions $\mathcal{E}$ pulled-back from $X_{A} \times X_{A}$ and $X_{B} \times X_{B}$. More precisely, we consider pairs $(L, \alpha)$, where $\alpha$ is a line bundle on $L$ that gives a lifting of $L$ to the Heisenberg groupoid of $X_{A} \times X_{B}$. As above, with each pair $(L, \alpha)$ we associate the LI-sheaf $S_{L, \alpha}$ on $A \times B$. Then we define the corresponding LI-functor $\Phi_{L, \alpha}: D^{b}(A) \rightarrow D^{b}(B)$ as the Fourier-Mukai functor associated with $S_{L, \alpha}$. Our main result is the calculation of the composition of functors of this form. We do this in two steps. First, we introduce generalized Lagrangians in $X_{A}$ by allowing for $Y$ not to be connected and for the homomorphism $Y \rightarrow X_{A}$ to have finite kernel. The condition for $Y$ to be Lagrangian is formulated in terms of duality between $Y$ and the complex $\left[Y \rightarrow X_{A}\right]$. The context for such a duality is provided by the category of orbi-abelian varieties studied in [23, Sec. 4]. The theory of invariants of Lagrangians in $D^{b}(A)$ carries over to generalized Lagrangians. We define composition of generalized Lagrangian correspondences (which is again generalized Lagrangian) and show that the 
composition of LI-functors is compatible with the composition of correspondences up to a shift (see Theorem 3.2.14). The second step consists of the study of the relation between the LI-sheaf $S_{Y, \alpha}$ associated with a generalized Lagrangian $(Y, \alpha)$ for $X_{A}$ and the corresponding usual Lagrangian obtained by passing to the image in $X_{A}$ of the connected component of zero $Y_{0} \subset Y$ (see Proposition 2.4.7). The final result (in characteristic zero) is that the composition of two LI-functors decomposes as an explicit direct sum of LI-functors. In the case $B=A$ we obtain an explicit description of a certain semiring of endofunctors of $D^{b}(A)$ in terms of the group $U\left(X_{A}, \mathbb{Q}\right)$ of symplectic automorphisms of $X_{A}$ in $\mathcal{A} b_{\mathbb{Q}}$. Considering these endofunctors up to a natural action of $X_{A}(k)$ we get a simpler picture: the resulting monoid has a group completion that can be identified with an explicit central extension of $U\left(X_{A}, \mathbb{Q}\right)$ by $\mathbb{Z} \times \mathbb{Q}^{*}$. The $\mathbb{Z}$-part of this central extension is described by essentially the same 2-cocycle as in the picture with autoequivalences of $D^{b}(A)$ (see $\left[18\right.$, Sec. 4]). The $\mathbb{Q}^{*}$-part of the central extension is a new feature of the picture with endofunctors, and we show that it can be nontrivial in the case of elliptic curves with complex multiplication.

The paper is organized as follows. In Section 1 we gather necessary results about finite Heisenberg group schemes and twisted equivariant sheaves, along with some other background facts. In Section 2 we review the symplectic setting from [20]. The main object here is the Heisenberg groupoid $\mathbf{H}$ associated with a symplectically self-dual abelian variety (equipped with some extra data). We define generalized isotropic (resp., Lagrangian) pairs and the corresponding representations of $\mathbf{H}$. The main result of this section is Theorem 2.4.5 describing the subcategories of invariants of a generalized Lagrangian pair in standard representations of $\mathbf{H}$. Another important technical result is Proposition 2.4.7 giving a relation between invariants for a generalized Lagrangian $i: Y \rightarrow X$ and the associated Lagrangian $i\left(Y_{0}\right) \subset X$. In Section 3 we study LI-kernels and LI-functors. First, we define generalized Lagrangian correspondences (or $g$-Lag-correspondences) and show that they can be composed under some assumptions. Then in 3.2 we prove the compatibility between the composition of g-Lag-correspondences and the composition of the corresponding LI-functors up to a shift (see Theorems 3.2.11 and Theorem 3.2.14). Finally in 3.3 assuming that the characteristic is zero we describe a semiring of endofunctors of $D^{b}(A)$ built from LI-functors and the related central extension of the group $U\left(X_{A}, \mathbb{Q}\right)$.

Notation and conventions: We consider schemes over a fixed algebraically closed ground field $k$. We say that an object $F$ of a $k$-linear category $\mathcal{C}$ is endosimple if $\operatorname{Hom}_{\mathcal{C}}(F, F)=k$. For a scheme $X$ we denote by $D^{b}(X)$ the bounded derived category of coherent sheaves on $X$. We say that an object $F \in D^{b}(X)$ is cohomologically pure if there exists a coherent sheaf $H$ such that $F \simeq H[n]$ for $n \in \mathbb{Z}$. For $F_{1}, F_{2} \in D^{b}(X)$ we set $\operatorname{Hom}_{D^{b}(X)}^{*}\left(F_{1}, F_{2}\right)=$ $\bigoplus_{i} \operatorname{Hom}_{D^{b}(X)}\left(F_{1}, F_{2}[i]\right)$. We denote by $\mathcal{A} b_{\mathbb{Q}}$ the category of abelian varieties up to an isogeny (i.e., the localization of the category of abelian varieties over $k$ with respect to the class of isogenies). For a finite group scheme $G$ by a representation of $G$ on a vector space we always mean a finite-dimensional representation. For a group scheme $G$ we denote by $G_{0}$ the connected component of the neutral element in $G$ with the reduced scheme structure, and we set $\pi_{0}(G)=G / G_{0}$. All our biextensions are biextensions by $\mathbb{G}_{m}$. Most of the facts about biextensions we use are summarized in [22, II.10]. We always normalize the Poincaré line bundle $\mathcal{P}=\mathcal{P}_{A}$ on $A \times \hat{A}$ by requiring it to be trivial over 
$\{0\} \times \hat{A}$ and $A \times\{0\}$ (thus making it a biextension of $A \times \hat{A}$ ). For a line bundle $\mathcal{L}$ over a commutative group scheme $X$ we denote by $\Lambda(\mathcal{L})$ the line bundle on $X \times X$ given by $\Lambda(\mathcal{L})_{x, x^{\prime}}=\mathcal{L}_{x+x^{\prime}} \otimes \mathcal{L}_{x}^{-1} \otimes \mathcal{L}_{x^{\prime}}^{-1}$

\section{Preliminaries}

1.1. Finite Heisenberg group schemes. In this section we recall some facts about Heisenberg group schemes and their representations of weight one following [22, Sec. 12.2] (see also [12, Ch. V] and [21, Sec. 1,2]).

A finite Heisenberg group scheme $G$ is a central extension of a finite commutative group scheme $K$ by $\mathbb{G}_{m}$, such that the commutator pairing $K \times K \rightarrow \mathbb{G}_{m}$ is perfect, i.e., induces an isomorphism $K \simeq K^{*}$, where $K^{*}$ is the Cartier dual to $K$.

In general, when $G$ is a central extension of some group scheme $K$ by $\mathbb{G}_{m}$ we say that a finite-dimensional representation $V$ of $G$ is of weight one if $\mathbb{G}_{m} \subset G$ acts on $V$ via the identity character. All weight-1 representations of a finite Heisenberg group scheme are direct sums of several copies of the unique irreducible representation of weight one, called Schrödinger representation. Its dimension is equal to $|K|^{1 / 2}$, where $|K|$ is the order of $K$.

Sometimes it is convenient to describe a Heisenberg extension $G$ in terms of the line bundle $L$ over $K$ associated with $G$ viewed as a $\mathbb{G}_{m}$-torsor over $K$. The group law on $G$ corresponds to an isomorphism

$$
L_{x+y} \simeq L_{x} \otimes L_{y}
$$

of line bundles on $K \times K$. A representation of $G$ of weight one on a finite dimensional vector space $V$ can be described as a morphism $L \otimes V \rightarrow \mathcal{O}_{K} \otimes V$ of bundles over $K$ satisfying the natural compatibility with (1.1.1). We denote by $G-$ rep $_{1}$ the category of weight-1 representations of $G$.

Any subgroup $I \subset K$, isotropic with respect to the commutator pairing, can be lifted to a subgroup of $G$. Equivalently, there is a trivialization of $L$ over $I$, compatible with (1.1.1). Any maximal isotropic subgroup of $K$ has order $|K|^{1 / 2}$. The Schrödinger representation can be realized explicitly as follows. The space $H^{0}(K, L)$ has natural commuting left and right $G$-actions of weight one: the corresponding isomorphisms

$$
L \otimes H^{0}(K, L) \simeq \mathcal{O}_{K} \otimes H^{0}(K, L)
$$

are obtained from (1.1.1) by taking the push-forward with respect to the projection $K \times$ $K \rightarrow K$ to one of the factors. Now if $I \subset K$ is a maximal isotropic subgroup then we can lift it to a subgroup of $G$ and take invariants $H^{0}(K, L)^{I}$ with respect to the right action. This becomes an irreducible $G$-representation of weight one. Trivializing the line bundle $L$ over $K$ we can identify $H^{0}(K, L)^{I}$ with the subspace of functions $f$ on $K$ satisfying certain quasi-periodicity with respect to translations by $I$, which is the customary description of the Schrödinger representation. If $V$ is a Schrödinger representation of $G$ and $I \subset K$ is

a maximal isotropic subgroup with a lifting to $G$ then $V^{I}$ is one-dimensional. Hence, if $W$ is any weight-1 representation of $G$ then we have an isomorphism of $G$-representations (depending on a trivialization of $V^{I}$ ):

$$
W \simeq V_{4} \otimes W^{I} .
$$


Note that if $V$ is a Schrödinger representation of $G$ then $V^{*}$ is a Schrödinger representation of $G^{o p}$ (using the isomorphism $G \rightarrow G^{o p}: g \mapsto g^{-1}$ we can also view $V^{*}$ as a representation of $G$ of weight -1$)$. For the entire space $H^{0}(K, L)$ we have an isomorphism of $G \times G^{o p}$-modules

$$
H^{0}(K, L) \simeq V \otimes V^{*} .
$$

Often when we talk about an isotropic subgroup in $K$ lifted to $G$ we just say that $I$ is an isotropic subgroup in $G$. If $I$ is such a subgroup then the normalizer subgroup $N(I)$ contains $\mathbb{G}_{m}$ and $N(I) / \mathbb{G}_{m}=I^{\perp}$, the orthogonal complement to $I$ with respect to the commutator pairing. The quotient $N(I) / I$ is a Heisenberg extension of $I^{\perp} / I$. If $V$ is a Schrödinger representation of $G$ then the space $V^{I}$ of $I$-invariants is a Schrödinger representation of $N(I) / I$. Viewed as a representation of $I, V$ is a direct $\operatorname{sum}$ of $\operatorname{dim}\left(V^{I}\right)$ copies of the regular representation $H^{0}(I, \mathcal{O})^{*} \simeq H^{0}\left(I^{*}, \mathcal{O}\right)$. Hence, in the case when $I^{*}$ is reduced we get an isomorphism

$$
V \simeq \bigoplus_{\chi \in I^{*}} V^{I} \otimes \chi
$$

of $I$-representations.

1.2. 1-cocycles with values in Picard stacks and twisted equivariant sheaves. Let us recall briefly some definitions from [23, Sec. 3.4]. Let $X$ be a scheme, $G$ a group scheme acting on $X$. A 1-cocycle of $G$ with values in the Picard stack Pic $(X)$ of line bundles on $X$ is a line bundle $L$ on $G \times X$ equipped with an isomorphism

$$
L_{g_{1} g_{2}, x} \simeq L_{g_{1}, g_{2} x} \otimes L_{g_{2}, x}
$$

on $G \times G \times X$ such that the following diagram of isomorphisms on $G \times G \times G \times X$ is commutatve:

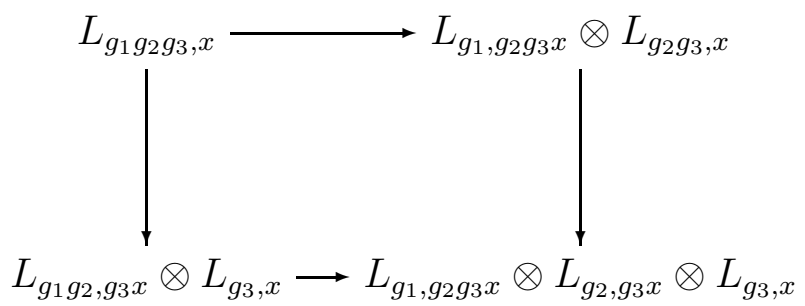

(cf. [23, (3.4.2)]). Given such a 1 -cocycle $L$ we can consider $L$-twisted $G$-equivariant sheaves on $X$ as sheaves equipped with an isomorphism

$$
F_{g x} \simeq L_{g, x} \otimes F_{x}
$$

on $G \times X$ subject to the commutativity of the diagram

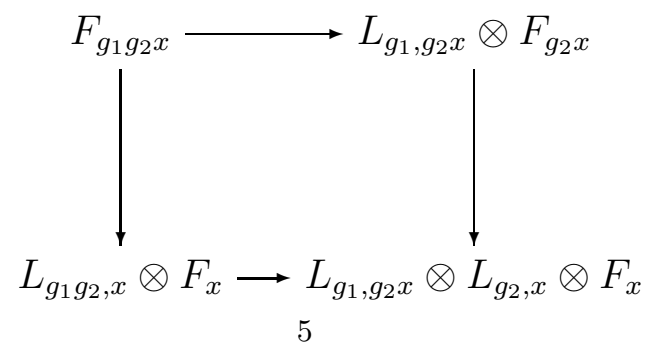


on $G \times G \times X$ (cf. [23, Sec. 3.4]). We denote the category of $L$-twisted $G$-equivariant coherent sheaves on $X$ by $\operatorname{Coh}_{H}^{L}(X)$. Similarly, we define the category $D_{H}^{L}(X)$ of $L$-twisted $G$-equivariant objects in the derived category $D^{b}(X)$ as objects $F \in D^{b}(X)$ equipped with an isomorphism (1.2.3) in $D^{b}(G \times X)$, subject to the same compatibilities.

For example, if $G \rightarrow K$ is Heisenberg group extension then the underlying $\mathbb{G}_{m}$-torsor $L$ over $K$ has a structure of a 1-cocycle of $K$ with values in the Picard stack $\mathcal{P} i c(k)$ of 1-dimensional vector spaces, and the category $G$ - rep 1 can be identified with the category of $L$-twisted $K$-equivariant coherent sheaves on $\operatorname{Spec}(k)$.

Lemma 1.2.1. Let $G$ be a central extension of a finite commutative group scheme $K$ by $\mathbb{G}_{m}$ with the center $C \times \mathbb{G}_{m}$, such that the induced central extension $\bar{G}=G / C \rightarrow K / C$ is a Heisenberg group. Let $p_{\bar{G}}: G \rightarrow \bar{G}$ and $p_{K}: G \rightarrow K$ be the projections, and let $V$ be a Schrödinger representation of $\bar{G}$. Then there exists an equivalence

$$
\Phi: G-\operatorname{rep}_{1} \rightarrow C-\text { rep }
$$

such that $\Phi\left(p_{G}^{*} V\right)$ is the trivial one-dimensional representation of $C$, and for a character $\chi: K \rightarrow \mathbb{G}_{m}$ we have

$$
\left.\Phi\left(\kappa_{\chi}^{*} W\right) \simeq \chi\right|_{C} \otimes \Phi(W)
$$

where $\kappa_{\chi}: G \rightarrow G$ is the automorphism sending $g$ to $\chi\left(p_{K}(g)\right) \cdot g$.

Proof. By [23, Lem. 3.5.3(i)], the extension of commutative groups

$$
0 \rightarrow C \rightarrow K \stackrel{\pi}{\longrightarrow} K / C \rightarrow 0
$$

gives rise to a biextension $\mathcal{B}$ of $K / C \times C^{*}$. Furthermore, if $\widetilde{\mathcal{M}}$ is the 1 -cocycle of $K$ with values in $\mathcal{P} i c(k)$ corresponding to the extension $G \rightarrow K$, then we have $\widetilde{\mathcal{M}} \simeq \pi^{*} \mathcal{M}_{0}$, where $\mathcal{M}_{0}$ is the 1 -cocycle of $K / C$ with values in $\mathcal{P} i c(k)$ corresponding to the extension $\bar{G} \rightarrow K / C$. We can extend $\mathcal{M}_{0}$ to a 1 -cocycle $\mathcal{M}$ of $K / C$ with values in $\operatorname{Pic}\left(C^{*}\right)$ by the formula

$$
\mathcal{M}_{x, c^{*}}=\mathcal{B}_{x, c^{*}} \otimes\left(\mathcal{M}_{0}\right)_{x}
$$

where $x \in K / C, c^{*} \in C^{*}$. Then one has a natural isomorphism of $k$-algebras

$$
\alpha: p_{*} \mathcal{O}_{C^{*}}[K / C]^{\mathcal{M}} \stackrel{\sim}{\longrightarrow} k[K]^{\widetilde{\mathcal{M}}},
$$

where $p: C^{*} \rightarrow \operatorname{Spec}(k)$ is the projection to the point (see [23, Prop. 3.5.4] for a more general result). Here for a group $H$ acting trivially on a scheme $X$ and a 1-cocycle $\mathcal{L}$ of $H$ with values in $\mathcal{P} i c(X)$ we denote by $\mathcal{O}_{X}[H]^{\mathcal{L}}$ the twisted group algebra of $H$ over $X$ (see $[23,(3.4 .9)]$ ). The equivalence between the categories of modules induced by $\alpha$ can be interpreted as an equivalence

$$
\operatorname{Coh}_{K / C}^{\mathcal{M}}\left(C^{*}\right) \simeq \operatorname{Coh}_{K}^{\widetilde{\mathcal{M}}}(\operatorname{Spec}(k))
$$

As we have seen above, the category on the right can be identified with $G-\operatorname{rep}_{1}$. On the other hand, $\operatorname{Coh}_{K / C}^{\mathcal{M}}\left(C^{*}\right)$ is equivalent to the category of weight-1 representations of the finite Heisenberg group scheme $\overline{\mathcal{G}}$ over $C^{*}$, where $\overline{\mathcal{G}}$ is the extension of the constant finite group scheme $(K / C)_{C^{*}}$ by $\mathbb{G}_{m}$ associated with the 1-cocycle $\mathcal{M}$. Since the obstacle to the existence of a Schrödinger representation is an element of the Brauer group $\operatorname{Br}\left(C^{*}\right)=0$ 
(see [21, Sec. 2]), the latter category is equivalent to $\operatorname{Coh}\left(C^{*}\right) \simeq C$-rep. Thus, we obtain the required equivalence (1.2.5).

It is easy to check that $\alpha$ is compatible with natural homomorphisms of both sides to $k[K / C]^{\mathcal{M}_{0}}$. Note that modules over $k[K / C]^{\mathcal{M}_{0}}$ are exactly weight-1 representations of $\bar{G}$. This compatibility implies that $p_{\bar{G}}^{*} V$ corresponds to the trivial representation of $C$ under the equivalence $\Phi$. The compatibility (1.2.6) with automorphisms $\kappa_{\chi}: G \rightarrow G$ follows from the fact that the whole construction is functorial with respect to isomorphisms $G \rightarrow G^{\prime}$, identical on $\mathbb{G}_{m}$.

We will use the following description of the categories of twisted equivariant sheaves associated with some transitive actions.

Proposition 1.2.2. Let $H$ be a group scheme acting transitively on $X$ with finite stabilizers, and let $L_{h, x}$ be a 1-cocycle for the action of $H$ on $X$.

(i) For a point $x \in X$ there is a natural central extension

$$
1 \rightarrow \mathbb{G}_{m} \rightarrow G_{x} \rightarrow H_{x} \rightarrow 1
$$

of the stabilizer subgroup $H_{x}$ of $x$ with the underlying line bundle $\left.L\right|_{H_{x} \times\{x\}}$. Consider the line bundle $L(x):=\left.L\right|_{H \times\{x\}}$ over $H$, and let $\pi(x): H \rightarrow X$ be the map sending $h$ to $h x$. Then $L(x)$ has a natural $\left(\mathrm{id}_{H} \times \pi(x)\right)^{*} L$-twisted $H$-equivariant structure with respect to the regular left action of $H$ on itself. The right action of $H_{x}$ on $H$ extends to an action of $G_{x}$ on $L(x)$ respecting the above twisted $H$-equivariant structure.

The map $\left.F \mapsto F\right|_{x}$ extends to an equivalence

$$
\mathrm{fib}_{x}: \operatorname{Coh}_{H}^{L}(X) \rightarrow G_{x}-\operatorname{rep}_{1}
$$

of L-twisted $H$-equivariant coherent sheaves on $X$ with the category of weight-1 representations of $G_{x}$.

(ii) For an element $h \in H$ the isomorphism $H_{x} \rightarrow H_{h x}$ given by the conjugation by $h$ extends to an isomorphism of central extensions

$$
\kappa_{h}: G_{x} \rightarrow G_{h x}
$$

Furthermore, there is a natural isomorphism of functors

$$
L_{h, x} \otimes \mathrm{fib}_{x} \simeq \kappa_{h}^{*} \circ \mathrm{fib}_{h x},
$$

where $\kappa_{h}^{*}: G_{g x}-\operatorname{rep}_{1} \rightarrow G_{x}-\operatorname{rep}_{1}$ is the equivalence induced by $\kappa_{h}$ ( $L_{h, x}$ is just a one-dimensional vector space one has to insert to make the isomorphism canonical).

(iii) Now let $f: X \rightarrow X$ be an automorphism commuting with the $H$-action. Let $M$ be a line bundle on $X$ together with an isomorphism of cocycles

$$
\tau_{f}: M_{x} \otimes M_{h x}^{-1} \otimes L_{h, x} \rightarrow L_{h, f(x)}
$$

(so $M$ is a coboundary for $\left.L \otimes(\mathrm{id} \times f)^{*} L^{-1}\right)$. Then the map $F \mapsto f^{*} F \otimes M$ extends to an autoequivalence $\iota_{f, M}$ of $\operatorname{Coh}_{H}^{L}(X)$, so that the following diagram of functors is commutative 
up to an isomorphism

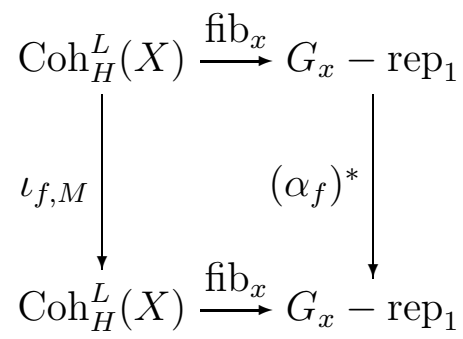

where $\alpha_{f}$ is the composition

$$
G_{x} \stackrel{\bar{\tau}_{f}}{\longrightarrow} G_{f(x)} \stackrel{\kappa_{h_{0}}^{-1}}{\longrightarrow} G_{x}
$$

for some $h_{0} \in H$ such that $h_{0} x=f(x)$. Here the homomorphism $\bar{\tau}_{f}$ is given by the restriction of $\tau_{f}$ to $H_{x} \times\{x\}$.

Proof. (i) Since the embedding of the point $x$ into $X$ is an $H_{x}$-morphism, the restriction of $\left.L\right|_{H_{x} \times\{x\}}$ has a natural structure of a 1-cocycle of $H_{x}$ with values in the Picard groupoid of the point, hence it defines a central extension of $H_{x}$ by $\mathbb{G}_{m}$. The $\left(\operatorname{id}_{H} \times \pi(x)\right)^{*} L$-twisted $H$-equivariant structure on $L(x)$ is given by the isomorphism

$$
L(x)_{h^{\prime} h} \simeq L_{h^{\prime}, \pi(x)(h)} \otimes L(x)_{h},
$$

where $h, h^{\prime} \in H$, obtained from (1.2.1) by the restriction to $H \times H \times\{x\}$. Restricting (1.2.1) further to $H \times H_{x} \times\{x\}$ we obtain an isomorphism

$$
L(x)_{h h_{0}} \simeq L(x)_{h} \otimes L_{h_{0}, x},
$$

where $h \in H, h_{0} \in H_{x}$. This isomorphism can be interpreted as a right action of $G_{x}$ on $L(x)$. The fact that this action commutes with the twisted left $H$-equivariant structure on $L(x)$ follows from the commutativity of diagram (1.2.2).

For an $L$-twisted $H$-equivariant coherent sheaf $F$ on $X$ we can restrict the structure isomorphism

$$
F_{h x} \simeq L_{h, x} \otimes F_{x}
$$

to $H_{x} \times\{x\} \subset H \times X$ and get an isomorphism

$$
F_{x} \stackrel{a\left(h_{0}, x\right)}{\longrightarrow} L_{h_{0}, x} \otimes F_{x}
$$

for $h_{0} \in H_{x}$ satisfying the natural compatibility condition on $H_{x} \times H_{x}$. Thus, we can view $F_{x}$ as a weight-1 representation of $G_{x}$. This gives the functor fib ${ }_{x}$.

Conversely, starting with a representation $W$ of $G_{x}$ of weight 1 we can construct an $L$-twisted $H$-equivariant sheaf on $X$ as follows. Consider the sheaf $\widetilde{F}=W \otimes L(x)$ on $H$. Viewing $W$ as a right module over $G_{x}$ of weight -1 (via the inversion map on $G_{x}$ ) and taking the tensor product of this action with the right action of $G_{x}$ on $L(x)$, we obtain a right action of $H_{x}$ on $\widetilde{F}$. Hence, $\widetilde{F}$ descends to a sheaf on $H / H_{x} \simeq X$. Now the $\left(\operatorname{id}_{H} \times \pi(x)\right)^{*} L$-twisted $H$-equivariant structure on $L(x)$ induces an $L$-twisted $H$-equivariant structure on $F$.

(ii) The cocycle structure on $L$ gives an isomorphism

$$
L_{h h_{0} h^{-1}, h x} \otimes L_{h, x} \simeq L_{h h_{0}, x}
$$


for $h, h_{0} \in H$. Combining it with the isomorphism

$$
L_{h, h_{0} x} \otimes L_{h_{0}, x} \simeq L_{h h_{0}, x}
$$

we get an isomorphism

$$
L_{h h_{0} h^{-1}, h x} \otimes\left(L_{h, x} \otimes L_{h, h_{0} x}^{-1}\right) \simeq L_{h_{0}, x}
$$

for fixed $h \in H$, compatible with the cocycle structures in $\left(h_{0}, x\right)$. The restriction to $h_{0} \in H_{x}$ gives an isomorphism

$$
L_{h h_{0} h^{-1}, h x} \simeq L_{h_{0}, x},
$$

compatible with the central extension structures, so we obtain the required isomorhism (1.2.9).

Now (1.2.10) is given by the isomorphism

$$
\phi: L_{h, x} \otimes F_{x} \stackrel{\sim}{\longrightarrow} F_{h x}
$$

which is a part of the $L$-twisted $H$-equivariant structure on $F$. To check the compatibility of $\phi$ with the $G_{x}$-action via the isomorphism (1.2.9), let us apply the commutativity of (1.2.4) for the pairs $\left(h, h_{0}\right)$ and $\left(h h_{0} h^{-1}, h\right)$, where $h_{0} \in H_{x}$. We get the following commutative diagram (in which we skipped the tensor product signs for brevity)

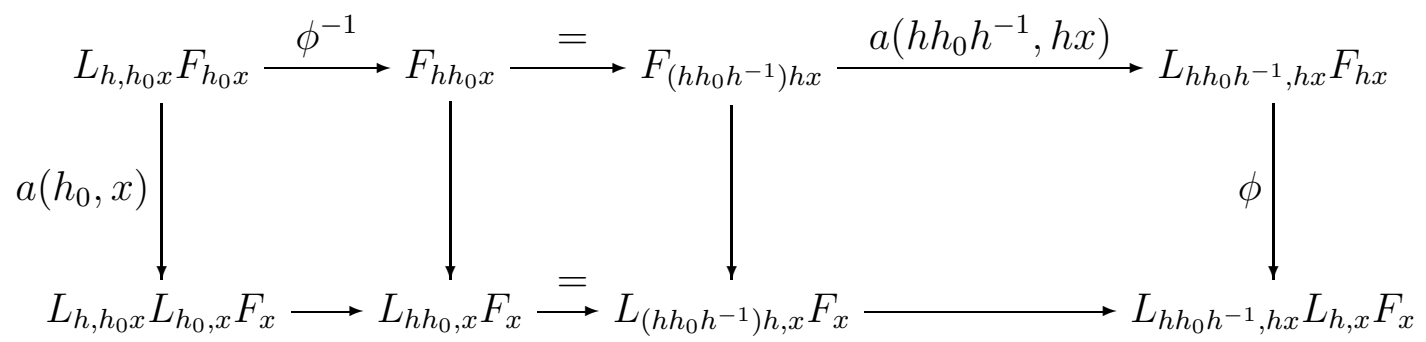

where $a(\cdot, \cdot)$ are the maps (1.2.13) inducing the action of $G_{x}$ (resp., $G_{h x}$ ) on $F_{x}$ (resp., $\left.F_{h x}\right)$. The required compatibility follows from the fact that the composition of arrows in the bottom row corresponds to the isomorphism (1.2.14).

(iii) The functoriality of the construction gives the commutative diagram

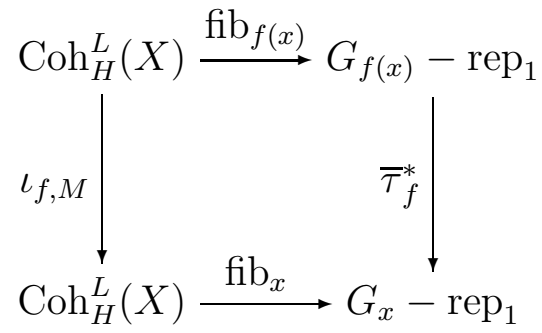

The diagram (1.2.11) follows by applying the isomorphism of functors established in (ii).

Lemma 1.2.3. In the situation of Proposition 1.2.2(i) the bundle

$$
\mathcal{V}=\pi(x)_{*}\left(\left.L\right|_{H \times\{x\}}\right)
$$

on $X$ has a natural $L$-twisted $H$-equivariant structure and a commuting right $G_{x}$-action (where $G_{x}$ acts trivially on $X$ ). Assume in addition that $H_{x}$ is commutative and $G_{x}$ 
is its Heisenberg extension. Let $I \subset G_{x}$ be a maximal isotropic subgroup. Then the bundle $\mathcal{V}^{I}$ of $I$-invariants is a simple object in $\operatorname{Coh}_{H}^{L}(X)$ (i.e., fib ${ }_{x}\left(\mathcal{V}^{I}\right)$ is a Schrödinger representation). Also, one has the following isomorphism of sheaves on $H$ compatible with the right $H_{x}$-action:

$$
\left.\pi(x)^{*} \mathcal{V}^{I} \simeq V \otimes L\right|_{H \times\{x\}}
$$

where $V$ is a Schrödinger representation of $G_{x}$, viewed as a representation of $G_{x}^{\text {op }}$ of weight -1 .

Proof. The $L$-twisted $H$-equivariant structure and the right $G_{x}$-action on $\mathcal{V}=\pi(x)_{*}(L(x))$ are induced by the $\left(\mathrm{id}_{H} \times \pi(x)\right)^{*} L$-twisted $H$-equivariant structure and the right $G_{x}$-action on $L(x)$, respectively. In the case when $G_{x}$ is a Heisenberg extension of $H_{x}$ and $I \subset G_{x}$ is a maximal isotropic subgroup, we have an isomorphism

$$
\operatorname{fib}_{x}\left(\mathcal{V}^{I}\right) \simeq H^{0}\left(H_{x},\left.L\right|_{H_{x} \times\{x\}}\right)^{I}
$$

compatible with the left $G_{x^{-}}$action. But the right-hand side is a standard model for the Schrödinger representation of $G$ (see Sec. 1.1). Hence, $\mathcal{V}^{I}$ is simple in $\operatorname{Coh}_{H}^{L}(X)$.

Let $V$ be a Schrödinger representation of $G_{x}$. Viewing $V^{*}$ as a Schrödinger representation of $G_{x}^{o p}$, we obtain an isomorphism

$$
\mathcal{V} \simeq V^{*} \otimes \mathcal{V}^{I}
$$

compatible with $L$-twisted $H$-equivariant structures and the right $G_{x}$-actions. Note also that we have a natural isomorphism $H \times_{X} H \simeq H \times H_{x}$ (where in the first product we use the map $\pi(x): H \rightarrow X)$. This gives an isomorphism

$$
\pi(x)^{*} \mathcal{V}=\pi(x)^{*} \pi(x)_{*}(L(x)) \simeq p_{H *} m^{*}(L(x)),
$$

where $m: H \times H_{x} \rightarrow H$ is given by the group law and $p_{H}: H \times H_{x} \rightarrow H$ is the projection. Taking into account the isomorphism (1.2.12) we get

$$
\pi(x)^{*} \mathcal{V}=H^{0}\left(H_{x},\left.L(x)\right|_{H_{x}}\right) \otimes L(x) .
$$

Hence, we obtain an isomorphism

$$
V^{*} \otimes \pi(x)^{*} \mathcal{V}^{I} \simeq \pi(x)^{*} \mathcal{V} \simeq H^{0}\left(H_{x},\left.L(x)\right|_{H_{x}}\right) \otimes L(x) \simeq V^{*} \otimes V \otimes L(x)
$$

compatible with $G_{x} \times G_{x}^{o p}$-actions, which leads to (1.2.15).

Example 1.2.4. Let $A$ be an abelian variety, $\mathcal{L}$ a line bundle on $A$ (trivialized at zero) $K \subset A$ a finite subgroup such that $\phi_{\mathcal{L}}(K)=0$, where $\phi_{\mathcal{L}}: A \rightarrow \hat{A}$ is the homomorphism associated with $A$. Consider the biextension $\Lambda(\mathcal{L})=m^{*} \mathcal{L} \otimes p_{1}^{*} \mathcal{L}^{-1} \otimes p_{2}^{*} \mathcal{L}^{-1}$ on $A \times A$. The restriction $\left.\Lambda(\mathcal{L})\right|_{A \times K}$ is trivial, hence, $\Lambda(\mathcal{L})$ descends to a biextension $\mathcal{B}$ of $A \times A / K$. Now set

$$
L=p_{1}^{*} \mathcal{L} \otimes \mathcal{B} .
$$

It is easy to see that this line bundle on $A \times A / K$ has a structure of a 1-cocycle with respect to the action of $A$ on $A / K$. Hence, we are in the situation of Proposition 1.2.2 with $H=A$ and $X=A / K$. Taking $x=0 \in A / K$ we obtain a central extension $G$ of $K$ by $\mathbb{G}_{m}$ with the underlying $\mathbb{G}_{m}$-torsor $\left.\mathcal{L}\right|_{K}$ and the right $G$-action on $\pi_{*}(\mathcal{L})$, where $\pi: A \rightarrow A / K$ is the projection. One can check that if $K=K(\mathcal{L})=\operatorname{ker}\left(\phi_{\mathcal{L}}\right)$ then 
$G \simeq G(L)^{o p}$, the opposite of the Mumford group of $L$ (see [22, Sec. 12.2]). This explains the seeming discrepancy between (1.2.15) and [22, Prop. 12.7].

1.3. Index of a symmetric isogeny. Recall that with every line bundle $L$ on an abelian variety $A$ one associates a symmetric homomorphism

$$
\phi_{L}: A \rightarrow \hat{A}: a \mapsto t_{a}^{*} L \otimes L^{-1} \in \operatorname{Pic}^{0}(A) .
$$

Furthermore, this induces an isomorphism

$$
N S(A)_{\mathbb{Q}} \rightarrow \operatorname{Hom}(A, \hat{A})_{\mathbb{Q}}^{+}
$$

of the Neron-Severi group with the space of symmetric homomorphisms tensored with $\mathbb{Q}$. In the case when $\phi_{L}$ is an isogeny there exists a unique integer $i(L), 0 \leq i(L) \leq g=\operatorname{dim} A$, such that

$$
H^{i}(A, L)=0 \text { for } i \neq i(L), H^{i(L)}(A, L) \neq 0 .
$$

It is called the index of $L$ and can be computed as the number of positive roots of the polynomial $P(n)=\chi\left(L \otimes L_{0}^{n}\right)$, where $L_{0}$ is an ample line bundle on $A$ (see [16, III.16]). The index has the property

$$
i\left(L^{n}\right)=i(L) \text { for } n>0 .
$$

We say that an element $\phi \in \operatorname{Hom}(A, B)_{\mathbb{Q}}$ is an isogeny if it is invertible in $\mathcal{A} b_{\mathbb{Q}}$, or equivalently if $n \phi$ is a usual isogeny in $\operatorname{Hom}(A, B)$ for some $n>0$. Using the isomorphism (1.3.1), we can define the index of a symmetric isogeny $\phi \in \operatorname{Hom}(A, \hat{A})_{\mathbb{Q}}^{+}$by choosing $n>0$ such that $n \phi=\phi_{L}$ and setting $i(\phi)=i(L)$. For any isomorphism $\psi: B \rightarrow A$ in $\mathcal{A} b_{\mathbb{Q}}$ we have

$$
i(\hat{\psi} \phi \psi)=i(\phi)
$$

1.4. Orbi-abelian varieties. We will use a natural extension of duality of abelian varieties to some length-2 complexes of commutative group schemes. The setup is similar to the duality of 1-motives considered in $[4,8]$ and can be generalized to include them (see [23, Sec. 4.3]).

Let $\mathcal{G}_{k}^{p r}$ denote the abelian category of commutative proper group schemes over $k$ (in characteristic zero every such group is a product of an abelian variety and a finite group). There exists an exact duality functor

$$
\mathbb{D}: D^{b}\left(\mathcal{G}_{k}^{p r}\right)^{o p} \rightarrow D^{b}\left(\mathcal{G}_{k}^{p r}\right)
$$

such that $\mathbb{D}(A)=\hat{A}$ for an abelian variety $A, \mathbb{D}(G) \simeq G^{*}[1]$ for a finite group scheme $G$, and $\mathbb{D}^{2}=$ Id (see $[23$, Thm. 4.1.4]).

An orbi-abelian variety is an object $K \in D^{b}\left(\mathcal{G}_{k}^{p r}\right)$ with $H^{i} K=0$ for $i \notin\{-1,0\}$, such that $H^{-1} K$ is a finite group scheme. Every orbi-abelian variety can be represented by a complex of the form $[G \rightarrow X]$ (in degrees -1 and 0 ), where $X \in \mathcal{G}_{k}^{p r}$ and $G$ is a finite group scheme. With every orbi-abelian variety $K$ we can associate an abelian variety $H^{0}(K)_{0}$ and two finite groups: $H^{-1}(K)$ and $\pi_{0} H^{0}(K)$. If $K$ is an orbi-abelian variety then $\mathbb{D}(K)$ is also such and the corresponding abelian varieties are dual, while the finite groups $H^{-1}(K)$ and $\pi_{0} H^{0}(K)$ get interchanged and dualized (see [23, Lem. 4.2.1]). 
1.5. Kernels and functors. Recall that there is a principle originating from noncommutative geometry stating that "correct" functors between the derived categories of coherent sheaves on smooth projective varieties are given by Fourier-Mukai functors (see [17], [25] for some incarnations of this principle). Such functors have form

$$
\Phi_{K}: D^{b}(X) \rightarrow D^{b}(Y): F \mapsto R p_{2 *}\left(p_{1}^{*} F \otimes^{\mathbb{L}} K\right),
$$

where $K \in D^{b}(X \times Y), p_{1}: X \times Y \rightarrow X$ and $p_{2}: X \times Y \rightarrow Y$ are the projections. We will refer to $K$ as a kernel representing the functor $\Phi_{K}$.

The composition of functors corresponds to an operation on kernels that we call convolution. Namely, for $K \in D^{b}(X \times Y)$ and $K^{\prime} \in D^{b}(Y \times Z)$ we set

$$
K \circ_{Y} K^{\prime}=R p_{13 *}\left(p_{12}^{*} K \otimes^{\mathbb{L}} p_{23}^{*} K^{\prime}\right) \in D^{b}(X \times Z),
$$

where $p_{i j}$ are projections from $X \times Y \times Z$ to the products of two of the factors. This operation is associative and we have a natural isomorphism of functors

$$
\Phi_{K \circ{ }^{\prime} K^{\prime}} \simeq \Phi_{K^{\prime}} \circ \Phi_{K}
$$

(see e.g. [23, Sec. 2.1]).

\section{Representations of the Heisenberg Groupoid}

2.1. Symplectic setting for abelian varieties. This setting was introduced in [20] (see also [22], 15.2). The basic "symplectic object" we work with is an abelian variety $X$ equipped with an isomorphism $\eta: X \rightarrow \hat{X}$ such that $\hat{\eta}=-\eta$.

Definition 2.1.1. We will call $(X, \eta)$ as above a symplectically selfdual abelian variety (ss-abelian variety for short).

The corresponding analogue of a symplectic form is the skew-symmetric biextension $\mathcal{E}=(\text { id } \times \eta)^{*} \mathcal{P}_{X}$ of $X \times X$ obtained by the pull-back from the Poincaré line bundle $\mathcal{P}_{X}$ on $X \times \hat{X}$ (see [20]). Often we need to assume that there exists a biextension $\mathcal{B}$ on $X \times X$ such that $\mathcal{E}$ is obtained from $\mathcal{B}$ by antisymmetrization: $\mathcal{E} \simeq \mathcal{B} \otimes \sigma^{*} \mathcal{B}^{-1}$, where $\sigma: X \rightarrow X$ is the permutation of factors.

Definition 2.1.2. The data $(X, \eta, \mathcal{B})$ as above is called an enhanced symplectically selfdual abelian variety (or ess-abelian variety for short).

The main example of an ess-abelian variety is $X_{A}=A \times \hat{A}$, where $A$ is an abelian variety, and $\mathcal{B}=p_{14}^{*} \mathcal{P}$ on $X \times X=A \times \hat{A} \times A \times \hat{A}$ (here $\mathcal{P}=\mathcal{P}_{A}$ is the Poincaré line bundle on $A \times \hat{A}$ ). We will refer to this example as the standard ess-abelian variety associated with $A$.

We consider ess-abelian varieties as analogs of symplectic vector spaces. In [20] we introduced a natural analog of the Heisenberg group in this context. First, let us consider the case $X_{A}=A \times \hat{A}$. In this case for every point $x=(a, \xi) \in X_{A}(k)$ we have a functor

$$
T_{x}^{A}=\left(\otimes \mathcal{P}_{\xi}\right) \circ t_{a}^{*}: D^{b}(A) \rightarrow D^{b}(A),
$$

where $t_{a}: A \rightarrow A$ is the translation by $a \in A(k)$ and $\mathcal{P}_{\xi}=\left.\mathcal{P}\right|_{A \times\{\xi\}} \in \operatorname{Pic}^{0}(A)$ is the line bundle on $A$ corresponding to $\xi \in \hat{A}(k)$. These functors compose according to the 
canonical isomorphisms

$$
T_{x}^{A} \circ T_{x^{\prime}}^{A} \simeq \mathcal{B}_{x, x^{\prime}} \otimes T_{x+x^{\prime}}^{A} .
$$

To express the algebraic dependence of these functors on $x$ we can consider similar functors on $D^{b}(A \times S)$ depending on $x \in X_{A}(S)$, where $S$ is a $k$-scheme, and observe that the above isomorphisms still hold (and are compatible with pull-backs with respect to base changes $S^{\prime} \rightarrow S$ ). This motivates the following definition (see [20, Def. 2.1]).

Definition 2.1.3. Let $(X, \mathcal{B})$ be an ess-abelian variety. The Heisenberg groupoid $\mathbf{H}=$ $\mathbf{H}(X, \mathcal{B})$ associated with $(X, \mathcal{B})$ is the stack $B \mathbb{G}_{m} \times X$ equipped with the following structure of a stack of Picard groupoids: for a $k$-scheme $S$ define the functor $+: \mathbf{H}(S) \times \mathbf{H}(S) \rightarrow$ $\mathbf{H}(S)$ by

$$
(L, x)+\left(L^{\prime}, x^{\prime}\right)=\left(L \otimes L^{\prime} \otimes \mathcal{B}_{x, x^{\prime}}, x+x^{\prime}\right),
$$

where $L$ and $L^{\prime}$ are line bundles over $S$ and $x, x^{\prime} \in X(S)$.

We intend to consider actions of $\mathbf{H}$ on fibered categories by generalizing the example of the action of $\mathbf{H}\left(X_{A}\right)$ on the fibered category $S \mapsto D^{b}(A \times S)$ given by the functors $T_{x}^{A}$ above (such that $L \in B \mathbb{G}_{m}(S)$ acts by tensoring with the pull-back of $L$ to $A \times S$ ).

2.2. Isotropic and Lagrangian pairs. Similarly to the case of the classical Heisenberg group associated with a symplectic vector space we need Lagrangian subvarieties $Y$ in $X$ to define representations of $\mathbf{H}$. Below we generalize the setup of [20, Sec. 2] slightly in that we allow the map $Y \rightarrow X$ to have finite kernel.

Definition 2.2.1. A generalized isotropic pair $(Y, \alpha)$ consists of a commutative group scheme $Y$ equipped with a homomorphism $i: Y \rightarrow X$, such that $\operatorname{ker}(i)$ is finite, and of a line bundle $\alpha$ over $Y$ together with an isomorphism

$$
\Lambda(\alpha) \simeq(i \times i)^{*} \mathcal{B}
$$

of biextensions on $Y \times Y$ inducing a cube structure on $\alpha$. In the case when $\operatorname{ker}(i)$ is trivial we say that $(Y, \alpha)$ is an isotropic pair (see [20]).

The reason for this definition becomes clear in connection with the Heisenberg groupoid. Namely, the data $(Y, \alpha)$ as above determines a homofunctor of stacks of Picard groupoids

$$
Y \rightarrow \mathbf{H}: y \mapsto\left(\alpha_{y}, i(y)\right) .
$$

Note that if $(Y, \alpha)$ is a generalized isotropic pair then the biextension $(i \times i)^{*} \mathcal{E}$ is trivialized. Therefore, we can view $(i \times \mathrm{id})^{*} \mathcal{E}$ as a biextension of $Y \times[Y \rightarrow X]$. By $[23$, Prop. 4.3.2], this biextension gives a morphism

$$
Y \rightarrow \mathbb{D}[Y \rightarrow X]
$$

that fits into a morphism of exact triangles in the derived category $D^{b}\left(\mathcal{G}^{p r}\right)$

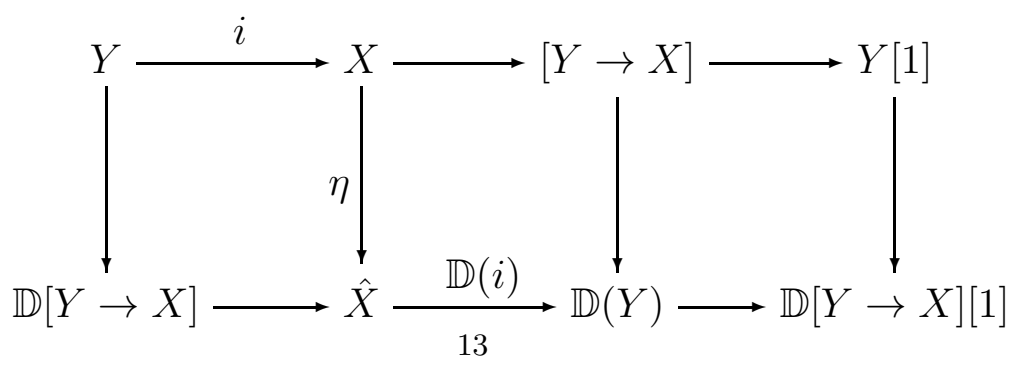


Conversely, if $i: Y \rightarrow X$ is such that the composition

$$
Y \stackrel{i}{\longrightarrow} X \stackrel{\eta}{\longrightarrow} \hat{X} \stackrel{\mathbb{D}(i)}{\longrightarrow} \mathbb{D}(Y)
$$

is zero then $Y$ extends to a generalized isotropic pair.

Definition 2.2.2. A generalized Lagrangian pair $(Y, a)$ is a generalized isotropic pair such that $(2.2 .2)$ is an isomorphism in $D^{b}\left(\mathcal{G}^{p r}\right)$. If $\operatorname{ker}(i)=0$ and $Y$ is connected then we say that $(Y, \alpha)$ is a Lagrangian pair and $Y$ is Lagrangian.

Lemma 2.2.3. (i) If $(Y, \alpha)$ is a generalized isotropic pair then $\operatorname{dim} Y \leq \operatorname{dim} X / 2$. If $(Y, \alpha)$ is generalized Lagrangian then $\operatorname{dim} Y=\operatorname{dim} X / 2$.

(ii) Let $Y \subset X$ be an isotropic abelian subvariety such that $\operatorname{dim} Y=\operatorname{dim} X / 2$. Then $Y$ is Lagrangian.

(iii) Let $(Y, \alpha)$ be a generalized Lagrangian pair. Then $Y$ is connected if and only if $\operatorname{ker}(i)=0$ if and only if $Y$ is Lagrangian.

Proof. (i) The first assertion follows from the fact that the composition

$$
Y_{0} \stackrel{i^{\prime}}{\longrightarrow} X \stackrel{\eta}{\longrightarrow} \hat{X} \stackrel{\hat{i}^{\prime}}{\longrightarrow} \widehat{Y}_{0}
$$

is zero and that $i^{\prime}=\left.i\right|_{Y_{0}}$ has finite kernel. If $(Y, \alpha)$ is generalized Lagrangian then we obtain from the isomorphism (2.2.2) the equality

$$
\operatorname{dim} Y=\operatorname{dim} H^{0} \mathbb{D}[Y \rightarrow X]=\operatorname{dim} H^{0}[Y \rightarrow X]=\operatorname{dim} X-\operatorname{dim} Y .
$$

(ii) Since $\eta(Y)$ and $\widehat{X / Y}$ are both abelian subvarieties in $\hat{X}$, it follows that $\eta(Y)=\widehat{X / Y}$ if and only if $\operatorname{dim} Y=\operatorname{dim} X / Y$.

(iii) This follows from the duality between the finite groups $H^{-1}[Y \rightarrow X]=\operatorname{ker}(i)$ and $\pi_{0} H^{0} \mathbb{D}[Y \rightarrow X] \simeq \pi_{0}(Y)$ (see [23, Lem. 4.2.1]).

Part (ii) of the above lemma shows that to determine whether an abelian subvariety of $X$ is Lagrangian we can work in the category of abelian varieties up to isogeny.

Examples 2.2.4. 1. Consider the standard ess-abelian variety $X_{A}=A \times \hat{A}$. Then an abelian subvariety $Y \subset X_{A}$, finite and surjective over $A$, gives rise to a morphism from $A$ to $\hat{A}$ in the category $\mathcal{A} b_{\mathbb{Q}}$ of abelian varieties up to an isogeny, namely, the morphism $f_{Y}=p_{\hat{A}} \circ p_{A}^{-1}$, where $p_{A}: Y \rightarrow A$ and $p_{\hat{A}}: Y \rightarrow A$ are projections. It is easy to see that the isotropy condition on $Y$ is equivalent to the condition that $f_{Y}$ is symmetric, i.e., $\hat{f}_{Y}=f_{Y}$. By Lemma 2.2.3(ii), this is equivalent to $Y$ being Lagrangian. Conversely, with every symmetric morphism $f \in \operatorname{Hom}(A \rightarrow \hat{A})_{\mathbb{Q}}$ we can associate its graph (which will be Lagrangian)

$$
\Gamma(f):=\{(N a, N f(a)) \mid a \in A\} \subset A \times \hat{A},
$$

where $N>0$ is an integer such that $N f \in \operatorname{Hom}(A, \hat{A})$. In this way we get a one-to-onecorrespondence between the set of Lagrangian subvarieties in $X_{A}$, finite over $A$, and the subspace $\operatorname{Hom}(A, \hat{A})_{\mathbb{Q}}^{+} \subset \operatorname{Hom}(A, \hat{A})_{\mathbb{Q}}$ of symmetric homomorphisms.

2. If $Y \subset X$ is a Lagrangian abelian subvariety then we can always choose a line bundle $\alpha$ such that $(L, \alpha)$ is a Lagrangian pair. Indeed, the biextension $\left.\mathcal{B}\right|_{Y \times Y}$ of $Y \times Y$ is symmetric, hence, it is of the form $\Lambda(\alpha)$ for some line bundle $\alpha$ on $Y$ (see e.g. [22, Thm. 13.7]). 
3. Let $X=0$. Then a generalized Lagrangian $(Y, \alpha)$ is a finite commutative group scheme $Y$ together with a central extension of $Y$ by $\mathbb{G}_{m}$ (given by $\alpha$ ), such that the corresponding commutator pairing $Y \times Y \rightarrow \mathbb{G}_{m}$ induces an isomorphism $Y \rightarrow Y^{*}$. In other words, this gives a finite Heisenberg group scheme.

Lemma 2.2.5. If $(Y, \alpha)$ is a generalized isotropic pair such that $\operatorname{dim} Y=\operatorname{dim} X / 2$ then $i\left(Y_{0}\right) \subset X$ is a Lagrangian abelian subvariety, where $Y_{0} \subset Y$ is the connected component of 0 in $Y$.

Proof. By definition, the composition $Y \rightarrow X \Im \hat{X} \rightarrow \mathbb{D}(Y)$ is zero. Hence, the same is true for the composition

$$
Y_{0} \rightarrow X \widetilde{\rightarrow} \hat{X} \rightarrow \widehat{Y}_{0}
$$

Since the natural morphisms $Y_{0} \rightarrow i\left(Y_{0}\right)$ and $\widehat{i\left(Y_{0}\right)} \rightarrow \widehat{Y}_{0}$ are isogenies, we derive that the composition

$$
i\left(Y_{0}\right) \rightarrow X \widetilde{\rightarrow} \hat{X} \rightarrow \widehat{i\left(Y_{0}\right)}
$$

is zero. By Lemma 2.2.3(ii), this implies that $i\left(Y_{0}\right)$ is Lagrangian.

Definition 2.2.6. Let us say that generalized Lagrangian pairs $(Y, \alpha)$ and $(Z, \beta)$ are transversal if the images of $Y$ and $Z$ generate $X$, or equivalently if $Y \times_{X} Z$ is finite.

Lemma 2.2.7. (i) For any pair of Lagrangian subvarieties $Y$ and $Z$ in $X$ there exists a Lagrangian subvariety $Q \subset X$ transversal to both $Y$ and $Z$.

(ii) Now assume in addition that $X=X_{A}$ (equipped with the standard symplectic biextension) and $Y=\{0\} \times \hat{A}$. Let $\mathcal{L}$ be an ample line bundle on $\hat{A}$. Then for almost all $n \in \mathbb{Z}$ (i.e., for all except for a finite number) the Lagrangian subvariety $\Gamma\left(n \phi_{\mathcal{L}}\right) \subset \hat{A} \times A=X_{A}$ (see Example 2.2.4.1) is transversal to $Z$.

Proof. (i) To prove the first assertion we can argue in the semisimple category $\mathcal{A} b_{\mathbb{Q}}$ of abelian varieties up to isogeny (since we have natural analogs of the relevant notions in $\left.\mathcal{A} b_{\mathbb{Q}}\right)$. In particular, in this category the condition of transversality of $Q$ to $Y$ and $Z$ becomes simply $Q \cap Y=Q \cap Z=0$. We can assume that $X \simeq Y \oplus \hat{Y}$, where both $Y$ and $\hat{Y}$ are Lagrangian in $X$ and the self-duality of $X$ restricts to the duality between $Y$ and $\hat{Y}$ (see [20, Lemma 1.1]). Let $T=Y \cap Z$. We can write

$$
Y=T \oplus P, \quad X=T \oplus P \oplus \hat{T} \oplus \hat{P},
$$

so that

$$
Z=\{(t, \phi(\xi), 0, \xi) \mid t \in T, \xi \in \hat{P}\},
$$

where $\phi: \hat{P} \rightarrow P$ is a symmetric morphism. Then choosing a symmetric isomorphism $\psi: \hat{P} \rightarrow P$ in $\mathcal{A} b_{\mathbb{Q}}$ we can set

$$
Q=\{(0,(\phi+\psi)(\xi), \eta, \xi) \mid \eta \in \hat{T}, \xi \in \hat{P}\} .
$$

(ii) As in part (i) we consider the decompositions (2.2.4) in $\mathcal{A} b_{\mathbb{Q}}$ (with $Y=\hat{A}$ ) so that (2.2.5) holds (still in $\mathcal{A} b_{\mathbb{Q}}$ ). Let us write the homomorphism $\phi_{\mathcal{L}}: Y \rightarrow \hat{Y}$ in the form

$$
\phi_{\mathcal{L}}=\left(\begin{array}{ll}
\alpha & \beta \\
\gamma & \delta
\end{array}\right),
$$


with $\alpha \in \operatorname{Hom}(T, \hat{T})_{\mathbb{Q}}, \beta \in \operatorname{Hom}(P, \hat{T})_{\mathbb{Q}}, \gamma \in \operatorname{Hom}(T, \hat{P})_{\mathbb{Q}}$ and $\delta \in \operatorname{Hom}(P, \hat{P})_{\mathbb{Q}}$. It is easy to see that the condition of transversality of $\Gamma\left(n \phi_{\mathcal{L}}\right)$ with $Z$ is equivalent to the condition that $n \delta-\phi$ is an isogeny (i.e., invertible in $\mathcal{A} b_{\mathbb{Q}}$ ). Note that some multiple of $\delta$ is the symmetric homomorphism $P \rightarrow \hat{P}$ corresponding to the restriction of $\mathcal{L}$ to $P$. Since this restriction is still ample, it follows that $\delta$ is an isogeny. Thus, $\operatorname{deg}(n \delta-\phi)$ is a polynomial in $n$ with nonzero top degree coefficient $\operatorname{deg}(\delta)$, so $\operatorname{deg}(n \delta-\phi) \neq 0$ for almost all $n \in \mathbb{Z}$.

The construction of the following proposition is a straightforward generalization of [22, Lem. 15.4] (see also [20, Sec. 3]). Recall that given a biextension $\mathcal{L}$ of $Y \times Z$ by $\mathbb{G}_{m}$ and a pair of homomorphisms $A \rightarrow Y, B \rightarrow Z$, together with trivializations of the pull-backs of $\mathcal{L}$ to $A \times Z$ and $Y \times B$, we obtain a pairing

$$
A \times B \rightarrow \mathbb{G}_{m}
$$

measuring the difference between two induced trivializations of the pull-back of $\mathcal{L}$ to $A \times B$ (cf. $[22,10.4])$.

Proposition 2.2.8. Let $(i: Y \rightarrow X, \alpha)$ and $(j: Z \rightarrow X, \beta)$ be generalized Lagrangian pairs such that $Y \times_{X} Z$ is finite. Then there is a natural central extension $G$ of $K=Y \times_{X} Z$ by $\mathbb{G}_{m}$ with the underlying line bundle $\beta_{z} \otimes \alpha_{y}^{-1}$ over $K$. Moreover, $G$ is a Heisenberg group scheme and the corresponding commutator form $K \times K \rightarrow \mathbb{G}_{m}$ is the pairing associated with the biextension $(i \times j)^{*} \mathcal{E}$ of $Y \times Z$ and with natural trivializations of its pull-backs to $K \times Z$ and $Y \times K$.

Proof. Pulling back the isomorphisms

$$
\Lambda(\alpha) \simeq(i \times i)^{*} \mathcal{B}, \quad \Lambda(\beta) \simeq(j \times j)^{*} \mathcal{B}
$$

(see (2.2.1)) to $K \times K$ we obtain an isomorphism $\Lambda\left(\beta_{z} \otimes \alpha_{y}^{-1}\right) \simeq \mathcal{O}_{K \times K}$ that gives a central extension structure on the corresponding $\mathbb{G}_{m}$-torsor $G \rightarrow K$. The isomorphisms (2.2.6) also induce trivializations of the pull-backs of $\mathcal{E}$ to $Y \times Y$ and to $Z \times Z$. Hence, we have natural trivializations of the pull-backs of $\mathcal{E}$ to $K \times Z$ and $Y \times K$ whose difference gives a pairing

$$
e: K \times K \rightarrow \mathbb{G}_{m}
$$

Note that $\mathcal{E}$ induces a duality between $Y$ and $[Y \rightarrow X] \simeq[K \rightarrow Z]$ and between $Z$ and $[Z \rightarrow X] \simeq[K \rightarrow Y]$. Thus, the dual of the exact triangle

$$
K \rightarrow Z \rightarrow[K \rightarrow Z] \rightarrow \ldots
$$

is the exact triangle

$$
Y \rightarrow[K \rightarrow Y] \rightarrow K[1] \rightarrow \ldots
$$

and we obtain an isomorphism $K \simeq K^{*}$. It is easy to see that it is given by the pairing $e$. Furthermore, the same reasoning as in [22, Lem. 15.4] shows that $e$ is the commutator form of the central extension $G \rightarrow K$. 
2.3. Representations associated with Lagrangian pairs and intertwining functors. Let $(Y, \alpha)$ be an isotropic pair. Then the data $\left(Y \rightarrow X,\left.\mathcal{B}\right|_{Y \times X}, \alpha\right)$ is similar to the twisting data considered in [23, Sec. 3.7] with the only difference that $Y$ is not a finite group scheme. In particular, we still have the 1-cocycle of $Y$ with values in $\operatorname{Pic}(X)$ defined by

$$
\mathcal{L}=\left.p_{1}^{*} \alpha^{-1} \otimes \mathcal{B}\right|_{Y \times X} ^{-1} .
$$

Recall that in $[20$, Sec. 2] we defined the category $\mathcal{F}(Y, \alpha)$ as the category of $\mathcal{L}$-twisted $Y$-equivariant objects in $D^{b}(X)$ (see Sec. 1.2). Explicitly, the objects of $\mathcal{F}(Y, \alpha)$ are objects $F \in D^{b}(X)$ equipped with isomorphisms

$$
F_{x+y} \simeq \alpha_{y}^{-1} \otimes \mathcal{B}_{y, x}^{-1} \otimes F_{x}
$$

on $X \times Y$, satisfying the natural cocycle condition on $X \times Y \times Y$.

For every $k$-scheme $S$ we can define similarly the category $\mathcal{F}(Y, \alpha)_{S}$ as the category of $\mathcal{L}$-twisted $Y$-equivariant objects in $D^{b}(X \times S)$, so that $S \mapsto \mathcal{F}(Y, \alpha)_{S}$ is a fibered category with respect to the natural pull-back functors. The Heisenberg groupoid $\mathbf{H}$ naturally acts on this fibered category. Namely, for every $S$ we have an action of $\mathbf{H}(S)$ on $\mathcal{F}(Y, \alpha)_{S}$ given by the functors

$$
T_{L, x}(F)_{x^{\prime}}=L \otimes \mathcal{B}_{x^{\prime}, x} \otimes F_{x+x^{\prime}},
$$

and these actions are compatible with the pull-backs with respect to morphisms $S \rightarrow S^{\prime}$. We also set $T_{x}=T_{\mathcal{O}_{S}, x}$ for $x \in X(S)$.

Remarks 2.3.1. 1. In the case when the projection $X \rightarrow X / Y$ has a section $s: X / Y \rightarrow$ $X$ there is a natural equivalence $\mathcal{F}(Y, \alpha) \simeq D^{b}(X / Y)$ obtained by restricting $F \in \mathcal{F}(Y, \alpha)$ to $s(X / Y) \subset X$. Furthermore, the functors $T_{L, x}$ are given by some kernels on $X / Y \times X / Y$, so we get a kernel representation in the sense of [23, Sec. 3.2] (see also Example 2.3.2 below).

2. The above definition of $\mathcal{F}(Y, \alpha)$ is not quite adequate in general. For example, it is not clear whether $\mathcal{F}(Y, \alpha)$ has a triangulated structure such that the embedding $\mathcal{F}(Y, \alpha) \rightarrow D^{b}(X)$ is an exact functor. If $\operatorname{char}(k)=0$ then $\mathcal{F}(Y, \alpha)$ can be identified with the (bounded) derived category of modules over some Azumaya algebra over $X / Y$ (see [20, Prop. 3.1]). This identification uses the derived descent with respect to finite flat morphisms (see $[20 \text {, Appendix }]^{1}$, [7]). A more adequate replacement for $\mathcal{F}(Y, \alpha)$ is the derived category of the abelian category $\mathcal{F}(Y, \alpha) \cap \operatorname{Coh}(X)=\operatorname{Coh}_{Y}^{\mathcal{L}}(X)$ (cr. Remark 2.3.5 below). However, we don't need this since we will mostly work with objects of $\mathcal{F}(Y, \alpha)$ that are cohomologically pure.

Example 2.3.2. Consider the standard ess-abelian variety $X_{A}=A \times \hat{A}$ with $\mathcal{B}=p_{14}^{*} \mathcal{P}$ and take $Y=\{0\} \times \hat{A} \subset X_{A}, \alpha=\mathcal{O}$. Then using the section $A \times\{0\} \subset X_{A}$ of the projection $X_{A} \rightarrow A$ we obtain an equivalence of the category $\mathcal{F}(Y, \alpha)$ with $D^{b}(A)$. One immediately checks that under this equivalence the functors $T_{x}$ giving the action of $\mathbf{H}(k)$ on $\mathcal{F}(Y, \alpha)$ become precisely the functors $T_{x}^{A}$ defined by (2.1.1). We will denote by $\mathbb{T}_{x}^{A} \in D^{b}(A \times A)$ the kernel giving the functor $T_{x}^{A}$. Explicitly, for $x=(a, \xi) \in A \times \hat{A}$,

$$
\mathbb{T}_{x}^{A}=\left(t_{a}, \operatorname{id}_{A}\right)_{*} \mathcal{P}_{\xi} .
$$

\footnotetext{
${ }^{1}$ The assumption $\operatorname{char}(k)=0$ was erroneously omitted in [20]
} 
Remark 2.3.3. Given an object $F \in D^{b}(A)$ we can consider the subset $Y_{F}$ of $x \in X_{A}$ such that $T_{x}^{A}(F) \simeq F$. Assuming that $F$ is endosimple and $\operatorname{Ext}^{i}(F, F)=0$ for $i<0$, one can check that $Y_{F}$ is an algebraic subgroup in $X_{A}$ and that we have a line bundle $\alpha$ on $Y_{F}$ and an isomorphism

$$
T_{y}^{A}(F) \simeq \alpha_{y} \otimes F
$$

over $Y_{F} \times A$ (this can be done using results of [9]; the case of vector bundles is considered in $\left[14\right.$, Sec. 1]). This easily implies that $\left(Y_{F}, \alpha\right)$ is an isotropic pair. In particular, $\operatorname{dim} Y_{F} \leq$ $\operatorname{dim} A$.

As in the classical case of the Heisenberg group, one expects that the representations $\mathcal{F}(Y, \alpha)$ associated with different Lagrangian pairs $(Y, \alpha)$ are equivalent. This can be proved under some additional assumptions (due to the need to use the derived descent, see Remark 2.3.1.2).

Theorem 2.3.4. Let $(Y, \alpha)$ and $(Z, \beta)$ be Lagrangian pairs for an ess-abelian variety $X$. Assume that either $\operatorname{char}(k)=0$ or $Y \cap Z=0$. Then there is an equivalence of fibered categories $\mathcal{F}(Y, \alpha)_{S} \simeq \mathcal{F}(Z, \beta)_{S}$ compatible with the $\mathbf{H}$-action.

Proof. In both cases the proof of [20, Thm. 4.3] works. The assumption $\operatorname{char}(k)=0$ allows one to use the derived descent, while in the case $Y \cap Z=0$ the descent problem does not arise.

Remark 2.3.5. In this paper we use only categories $\mathcal{F}(Y, \alpha)$ associated with Lagrangian pairs. However, the above equivalence can also be constructed for generalized Lagrangian pairs and the extra assumptions in Theorem 2.3.4 can be removed if we replace each category $\mathcal{F}(Y, \alpha)$ with the derived category of $\mathcal{F}(Y, \alpha) \cap \operatorname{Coh}(X)$ and use Theorem 4.5.1 of [23].

2.4. Invariants of a generalized Lagrangian pair. Consider the representation of $\mathbf{H}$ on the fibered category $S \mapsto \mathcal{F}(Y, \alpha)_{S}$, associated with a Lagrangian pair $(Y, \alpha)$. Now let $(j: Z \rightarrow X, \beta)$ be a generalized isotropic pair. Then we have a homofunctor

$$
\mathbf{j}: Z \rightarrow \mathbf{H}: z \mapsto\left(\beta_{z}, j(z)\right)
$$

of Picard stacks, so it makes sense to consider the category $\mathcal{F}(Y, \alpha)^{(Z, \beta)}$ of $(Z, \beta)$-invariants in $\mathcal{F}(Y, \alpha)$. By definition, the objects of this category are objects $F \in \mathcal{F}(Y, \alpha)$ equipped with a collection of isomorphisms

$$
T_{\mathbf{j}(z)}\left(F_{S}\right) \stackrel{\phi(z)}{\longrightarrow} F_{S}
$$

for all $k$-schemes $S$ and all $z \in Z(S)$, where $F_{S} \in \mathcal{F}(Y, \alpha)_{S}$ is the pull-back of $F$, compatible with pull-backs with respect to base changes $S \rightarrow S^{\prime}$ and satisfying the following cocycle condition

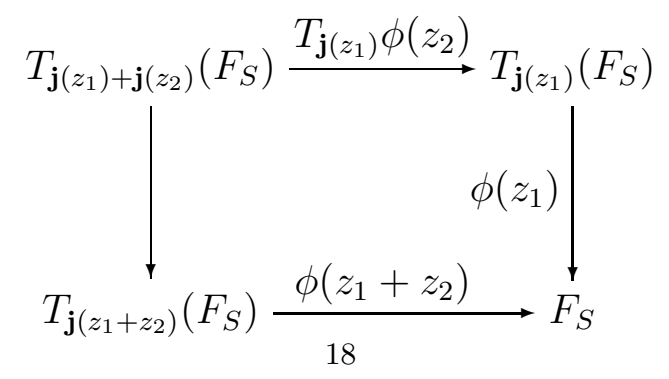


One can easily check that the collection $(\phi(z))$ is determined by the single element $\phi\left(z^{u n}\right)$, where $z^{\text {un }} \in Z(Z)$ is the tautological $Z$-point of $Z$. Thus, $\mathcal{F}(Y, \alpha)^{(Z, \beta)}$ can be also described as the category of objects $F \in \mathcal{F}(Y, \alpha)$ equipped with isomorphisms

$$
F_{j(z)+x} \simeq \beta_{z}^{-1} \otimes \mathcal{B}_{x, j(z)}^{-1} \otimes F_{x},
$$

over $Z \times X$, where $z \in Z, x \in X$, satisfying the natural cocycle condition over $Z \times Z \times X$ and the following compatibility for $F_{j(z)+x+y}$, where $y \in Y, z \in Z$ :

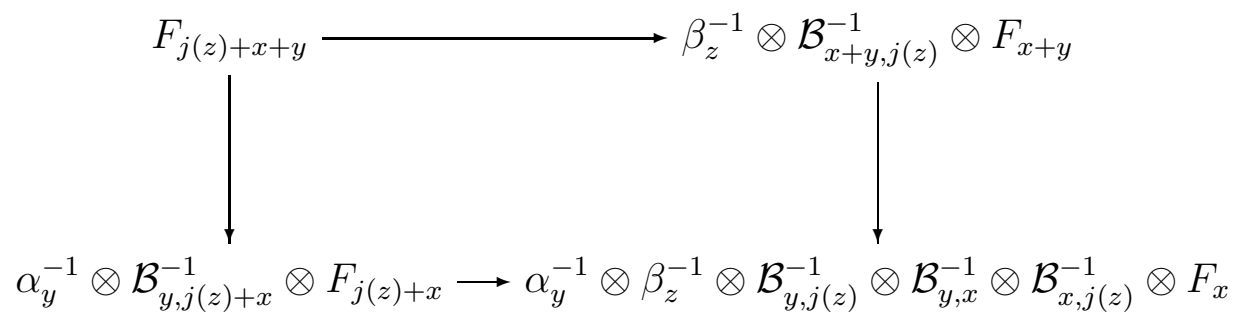

where the horizontal (resp., vertical) isomorphisms correspond to $(Z, \beta)$-invariance (resp., come from the definition of $\mathcal{F}(Y, \alpha))$.

Example 2.4.1. As in Example 2.3.2 let us consider the standard ess-abelian variety $X=X_{A}$ and $Y=\{0\} \times \hat{A} \subset X_{A}$, so that $\mathcal{F}(Y, \mathcal{O}) \simeq D^{b}(A)$. Then the skyscraper sheaf $k(0) \in D^{b}(A)$ is invariant with respect to $(Y, \mathcal{O})$. Now let $F$ be a nontrivial extension of $k(0)$ by itself, so $F$ is isomorphic to the structure sheaf of a length 2 subscheme $S \subset A$. Then the fact that the restriction of the Poincaré bundle to $S \times \hat{A}$ is nontrivial implies that $F$ is not $(Y, \mathcal{O})$-invariant, even though $F \otimes \mathcal{P}_{\xi} \simeq F$ for every $\xi \in \hat{A}(\xi)$. The Fourier dual of this example is a bundle $V$ which is a nontrivial extension of $\mathcal{O}_{A}$ by itself. The bundle $V$ is homogeneous, i.e., $t_{a}^{*} V \simeq V$ for every $a \in A$. However, it is not invariant with respect to $A \times\{0\} \subset X_{A}$ (and $\beta=\mathcal{O}$ ) according to our definition.

By Theorem 2.3.4, if $\left(Y^{\prime}, \alpha^{\prime}\right)$ is another Lagrangian pair then the categories $\mathcal{F}(Y, \alpha)^{Z, \beta}$ and $\mathcal{F}\left(Y^{\prime}, \alpha^{\prime}\right)^{Z, \beta}$ are equivalent, provided either $\operatorname{char}(k)=0$ or $Y \cap Y^{\prime}=0$. This observation will often allow us to reduce the study of $\mathcal{F}(Y, a)^{Z, \beta}$ to the case when $Y$ and $Z$ are transversal.

Lemma 2.4.2. Let $\beta^{\prime}=\left.\beta \otimes \mathcal{E}\right|_{Z \times\{x\}}$ for some $x \in X$ then the functor $T_{x}: \mathcal{F}(Y, \alpha) \rightarrow$ $\mathcal{F}(Y, \alpha)$ induces an equivalence

$$
t(Z, x): \mathcal{F}(Y, \alpha)^{\left(Z, \beta^{\prime}\right)} \rightarrow \mathcal{F}(Y, \alpha)^{(Z, \beta)} .
$$

Proof. Let $\mathbf{j}^{\prime}: Z \rightarrow \mathbf{H}: z \mapsto\left(\beta_{z}^{\prime}, j(z)\right)$ be the homofunctor associated with $\beta^{\prime}$. Then we have

$$
T_{\mathbf{j}^{\prime}(z)}=\mathcal{E}_{j(z), x} \otimes T_{\mathbf{j}(z)} .
$$

Hence, the structure of $\left(Z, \beta^{\prime}\right)$-invariance for $F \in \mathcal{F}(Y, \alpha)$ induces isomorphisms

$$
T_{\mathbf{j}(z)} T_{x}(F) \simeq \mathcal{E}_{j(z), x} \otimes T_{x} T_{\mathbf{j}(z)}(F) \simeq T_{x} T_{\mathbf{j}^{\prime}(z)}(F) \rightarrow T_{x}(F),
$$

i.e., the structure of $(Z, \beta)$-invariance for $T_{x}(F)$.

We are going to show that under some technical assumptions the category $\mathcal{F}(Y, \alpha)^{(Z, \beta)}$ is generated by a single coherent sheaf in the sense of the following definition. 
Definition 2.4.3. Let $\mathcal{T}$ be a triangulated category, $\mathcal{F} \subset \mathcal{T}$ an additive (not necessarily full) subcategory closed under shifts $X \mapsto X[i], i \in \mathbb{Z}$. For objects $F, F_{1}, F_{2} \in \mathcal{F}$ we say that $F$ is an $\mathcal{F}$-extension of $F_{2}$ by $F_{1}$ if there exists a triangle

$$
F_{1} \rightarrow F \rightarrow F_{2} \rightarrow F_{1}[1]
$$

in $\mathcal{F}$ which is an exact triangle in $\mathcal{T}$. We say that $F \in \mathcal{F}$ has an $\mathcal{F}$-filtration with successive quotients $Q_{1}, \ldots, Q_{n} \in \mathcal{F}$ if there exists a collection of objects $\left(F_{i j}\right), 0 \leq i<j \leq n$, in $\mathcal{F}$, such that $F_{i-1, i}=Q_{i}$ for $i=1, \ldots, n, F_{0 n}=F$, and for any $i<j<k, F_{i k}$ is an $\mathcal{F}$-extension of $F_{j k}$ by $F_{i j}$. We say that an object $F_{0} \in \mathcal{F}$ is a $t$-generator of $\mathcal{F}$ with respect to $\mathcal{T}$ if $\operatorname{Hom}_{\mathcal{T}}\left(F_{0}, F_{0}[i]\right)=0$ for $i<0$, and every nonzero object of $\mathcal{F}$ admits an $\mathcal{F}$-filtration with successive quotients $F_{0}^{\oplus m_{1}}\left[k_{1}\right], \ldots, F_{0}^{\oplus m_{n}}\left[k_{n}\right]$ for some $n>0$, where $k_{1}>k_{2}>\ldots>k_{n}$.

Note that the above definition of a $t$-generator mimics the situation when $\mathcal{F}$ has a $t$ structure with the heart consisting of direct sums of $F_{0}$, however, it uses the triangulated structure of an ambient category $\mathcal{T}$ (since $\mathcal{F}$ does not have to be triangulated).

Lemma 2.4.4. Let $F_{0}$ be a t-generator of $\mathcal{F}$ with respect to $\mathcal{T}$.

(i) Suppose $A \in \mathcal{F}$ (resp., $B \in \mathcal{F}$ ) has an $\mathcal{F}$-filtration with successive quotients

$$
F_{0}^{\oplus m_{1}}\left[a_{1}\right], \ldots, F_{0}^{\oplus m_{n}}\left[a_{n}\right] \quad \text { (resp., } F_{0}^{\oplus m_{1}^{\prime}}\left[b_{1}\right], \ldots, F_{0}^{\oplus m_{p}^{\prime}}\left[b_{p}\right] \text { ) }
$$

such that $a_{1}>\ldots>a_{n}$ (resp., $\left.b_{1}>\ldots>b_{p}\right)$. Then

$$
\operatorname{Hom}_{\mathcal{F}}\left(A, B\left[a_{n}-b_{1}\right]\right) \neq 0 .
$$

(ii) Let $F \in \mathcal{F}$ be an object such that $\operatorname{Hom}_{\mathcal{F}}(F, F[i])=0$ for $i<0$. Then $F \simeq F_{0}^{\oplus m}$ in $\mathcal{F}$. (iii) Suppose $F_{0}$ is an $\mathcal{F}$-extension of $B \in \mathcal{F}$ by $A \in \mathcal{F}$, where $\operatorname{Hom}_{\mathcal{T}}(A, B[i])=0$ for $i \leq 0$. Then either $A=0$ or $B=0$.

Proof. (i) By definition, we have an exact triangle

$$
A^{\prime} \rightarrow A \stackrel{\pi}{\longrightarrow} F_{0}^{\oplus m_{n}}\left[a_{n}\right] \rightarrow A^{\prime}[1]
$$

where $A^{\prime}$ has an $\mathcal{F}$-filtration with successive quotients of the form $F_{0}^{\oplus m}[i]$ such that $i>a_{n}$. Hence, $\operatorname{Hom}_{\mathcal{T}}\left(A^{\prime}[1], F_{0}\left[a_{n}\right]\right)=0$ and the map

$$
\operatorname{Hom}_{\mathcal{F}}\left(F_{0}^{\oplus m_{n}}\left[a_{n}\right], F_{0}\left[a_{n}\right]\right) \rightarrow \operatorname{Hom}_{\mathcal{F}}\left(A, F_{0}\left[a_{n}\right]\right)
$$

induced by $\pi$ is injective. In particular, $\operatorname{Hom}_{\mathcal{F}}\left(A, F_{0}\left[a_{n}\right]\right) \neq 0$. Next, let us consider an exact triangle

$$
F_{0}^{\oplus m_{1}^{\prime}}\left[a_{n}\right] \stackrel{\iota}{\longrightarrow} B\left[a_{n}-b_{1}\right] \rightarrow B^{\prime} \rightarrow \ldots
$$

where $B^{\prime}$ has an $\mathcal{F}$-filtration with successive quotients of the form $F_{0}^{\oplus m}[i]$, such that $i<a_{n}$. It follows that $\operatorname{Hom}_{\mathcal{T}}\left(A, B^{\prime}[-1]\right)=0$, so the map

$$
\operatorname{Hom}_{\mathcal{F}}\left(A, F_{0}^{\oplus m_{1}^{\prime}}\left[a_{n}\right]\right) \rightarrow \operatorname{Hom}_{\mathcal{F}}\left(A, B\left[a_{n}-b_{1}\right]\right)
$$

induced by $\iota$ is injective. Since the source space is non-zero, this implies the result.

(ii) We know that $F$ has an $\mathcal{F}$-filtration with successive quotients $F_{0}^{\oplus m_{1}}\left[k_{1}\right], \ldots, F_{0}^{\oplus m_{n}}\left[k_{n}\right]$ such that $k_{1}>\ldots>k_{n}$. Applying (i) to $A=B=F$ we deduce that $k_{n} \geq k_{1}$, so in fact, $n=1$. 
(iii) Assume that $A$ and $B$ have $\mathcal{F}$-filtrations as in (i). Then the result of (i) together with our vanishing assumption implies that $b_{1}<a_{n}$. On the other hand, in order to have a nonzero morphism $A \rightarrow F_{0}$ (resp., $F_{0} \rightarrow B$ ) we should have $a_{n} \leq 0$ (resp., $b_{1} \geq 0$ ). Thus, either $\operatorname{Hom}_{\mathcal{T}}\left(A, F_{0}\right)=0$ or $\operatorname{Hom}_{\mathcal{T}}\left(F_{0}, B\right)=0$. Assume that $\operatorname{Hom}_{\mathcal{T}}\left(A, F_{0}\right)=0$ (the second case is considered similarly). Then in $\mathcal{T}$ one has an isomorphism $B \simeq F_{0} \oplus A[1]$, so we obtain that

$$
\operatorname{Hom}_{\mathcal{T}}(A, B[-1])=\operatorname{Hom}_{\mathcal{T}}(A, A) \neq 0
$$

a contradiction.

Theorem 2.4.5. Let $(Y, \alpha)$ be a Lagrangian pair for an ess-abelian variety $X$, and let $(j: Z \rightarrow X, \beta)$ be a generalized Lagrangian pair for $X$. Assume that the following condition is satisfied:

( $\star$ ) either $Y$ and $Z$ are transversal, or $X=X_{A}$ and $Y=\{0\} \times \hat{A}$, or $\operatorname{char}(k)=0$. Then there exists a coherent sheaf

$$
S_{Z, \beta}=S_{Z, \beta}(Y, \alpha) \in \mathcal{F}(Y, \alpha)^{(Z, \beta)} \cap \operatorname{Coh}(X)
$$

which is a t-generator of $\mathcal{F}(Y, \alpha)^{(Z, \beta)}$ with respect to $\mathcal{F}(Y, \alpha)$. Furthermore, $S_{Z, \beta}$ is an endosimple object of $\mathcal{F}(Y, \alpha)^{(Z, \beta)}$. Every coherent sheaf in $\mathcal{F}(Y, \alpha)^{(Z, \beta)} \cap \operatorname{Coh}(X)$ is a direct sum of several copies of $S_{Z, \beta}$. In the case when $Y$ and $Z$ are transversal we have

$$
S_{Z, \beta}(Y, \alpha)=\left(s_{Y, Z}\right)_{*}\left(\alpha^{-1} \otimes \beta^{-1} \otimes(i \times j)^{*} \mathcal{B}^{-1}\right)^{I},
$$

where $i: Y \rightarrow X$ is the embedding map, $s_{Y, Z}: Y \times Z \rightarrow X$ is the natural map and $I$ is a maximal isotropic subgroup in $\operatorname{ker}\left(s_{Y, Z}\right) \simeq Y \times_{X} Z$ with respect to the commutator pairing from Proposition 2.2.8. We also have

$$
\operatorname{dim}_{k} \operatorname{End}_{\mathcal{F}(Y, \alpha)}\left(S_{Z, \beta}\right)=\left|\pi_{0}(Z)\right| .
$$

Proof. Assume first that $Y$ and $Z$ are transversal and let us show the existence of an endosimple $t$-generator in this case. Recall that an object $F \in \mathcal{F}(Y, \alpha)$ is equipped with an isomorphism

$$
F_{x+y} \simeq \alpha_{y}^{-1} \otimes \mathcal{B}_{y, x}^{-1} \otimes F_{x}
$$

where $y \in Y, x \in X$. On the other hand, the condition of $(Z, \beta)$-invariance is an isomorphism

$$
F_{j(z)+x} \simeq \beta_{z}^{-1} \otimes \mathcal{B}_{x, j(z)}^{-1} \otimes F_{x}
$$

where $z \in Z, x \in X$. Combining these two isomorphisms we get an isomorphism

$$
F_{j(z)+x+y} \simeq L_{(y, z), x} \otimes F_{x}
$$

over $(Y \times Z) \times X$, where

$$
L_{(y, z), x}=\alpha_{y}^{-1} \otimes \beta_{z}^{-1} \otimes \mathcal{B}_{x, j(z)}^{-1} \otimes \mathcal{B}_{y, j(z)}^{-1} \otimes \mathcal{B}_{y, x}^{-1} .
$$

has a natural structure of a 1-cocycle for the action of $Y \times Z$ on $X$ induced by the homomorphism $s_{Y, Z}: Y \times Z \rightarrow X$. Assume in addition that $F$ is a coherent sheaf. Then the compatibilities in the definition of $\mathcal{F}(Y, \alpha)^{(Z, \beta)}$ reduce to the condition that (2.4.3) gives $F$ a structure of an $L$-twisted $Y \times Z$-equivariant coherent sheaf on $X$. By Proposition 1.2.2, the category of such sheaves is equivalent to the category of weight one 
representations of the central extension $G$ of $K=\operatorname{ker}\left(s_{Y, Z}\right)$ by $\mathbb{G}_{m}$ given by the restriction $\left.L\right|_{K \times\{0\}}$. We have an identification

$$
\iota: Y \times_{X} Z \rightarrow K:(y, z) \mapsto(y,-z)
$$

such that

$$
\iota^{*}\left(\left.L\right|_{K \times\{0\}}\right)_{y, z} \simeq \alpha_{y}^{-1} \otimes \beta_{-z}^{-1} \otimes \mathcal{B}_{j(z),-j(z)}^{-1} \simeq \alpha_{y}^{-1} \otimes \beta_{j(z)},
$$

where the last isomorphism comes from the isomorphism $\Lambda(\beta) \simeq(j \times j)^{*} \mathcal{B}$. Hence, $G$ can be identified with the Heisenberg group of Proposition 2.2.8. Let us define $S_{Z, \beta} \in$ $\mathcal{F}(Y, \alpha)^{Z, \beta} \cap \operatorname{Coh}(X)$ as the sheaf corresponding to the Schrödinger representation of $G$. Note that it is an endosimple object of $\mathcal{F}(Y, \alpha)^{Z, \beta}$. The formula (2.4.1) follows immediately from Lemma 1.2.3. For any object $F \in \mathcal{F}(Y, \alpha)^{Z, \beta} \subset D^{b}(X)$ the triangles

$$
\tau_{\leq n-1} F \rightarrow F \rightarrow \tau_{\geq n} F \rightarrow \tau_{\leq n-1} F[1]
$$

(where $\tau_{\bullet}$ are the truncation functors associated with the standard $t$-structure) automatically belong to $\mathcal{F}(Y, \alpha)^{Z, \beta}$. Hence, the standard devissage of $F$ into its cohomology sheaves shows that $S_{Z, \beta}$ is a $t$-generator of $\mathcal{F}(Y, \alpha)^{Z, \beta}$.

The existence of an endosimple $t$-generator of $\mathcal{F}(Y, \alpha)^{Z, \beta}$ with respect to $\mathcal{F}(Y, \alpha)$ in the case when $Y$ and $Z$ are not necessarily transversal follows now from Theorem 2.3.4. More precisely, if $\operatorname{char}(k)=0$ then we can choose a Lagrangian pair $\left(Y^{\prime}, \alpha^{\prime}\right)$, transversal to $Z$ (see Lemma 2.2.7(i)) and use an equivalence of fibered categories $\mathcal{F}(Y, \alpha)_{S} \simeq \mathcal{F}\left(Y^{\prime}, \alpha^{\prime}\right)_{S}$ compatible with the $\mathbf{H}$-action. In the case when $Y=\{0\} \times \hat{A} \subset X_{A}=X$ we can assume that $Y^{\prime} \cap Y=0$ (see Lemma 2.2.7(ii)), so we again have an equivalence $\mathcal{F}(Y, \alpha)_{S} \simeq$ $\mathcal{F}\left(Y^{\prime}, \alpha^{\prime}\right)_{S}$.

Let us prove that such a $t$-generator $F_{0}$ of $\mathcal{F}(Y, \alpha)^{Z, \beta}$ with respect to $\mathcal{F}(Y, \alpha)$ is necessarily cohomologically pure. Suppose $F_{0}$ is not pure and consider the exact triangle (2.4.5) with $A=\tau_{\leq n-1} F_{0} \neq 0$ and $B=\tau_{\geq n} F_{0} \neq 0$. Then $\operatorname{Hom}(A, B[i])=0$ for $i \leq 0$, hence, the same space of morphisms in $\mathcal{F}(Y, \alpha)$ also vanishes. But this gives a contradiction with Lemma 2.4.4(iii).

Thus, $F_{0}$ can be shifted to become a coherent sheaf, and we set $S_{Z, \beta}=F_{0}$. The fact that every $F \in \mathcal{F}(Y, \alpha)^{(Z, \beta)} \cap \operatorname{Coh}(X)$ is a direct sum of several copies of $S_{Z, \beta}$ follows from Lemma 2.4.4(ii).

To prove (2.4.2) we can again assume that $Y$ and $Z$ are transversal. Let $V$ be a Schrödinger representation of the Heisenberg group scheme $G$ considered above. By Lemma 1.2.3, endomorphisms of $F=S_{Z, \beta}$ can be identified with $K$-invariants in endomorphisms of

$$
\left.s_{Y, Z}^{*} F \simeq V \otimes L\right|_{Y \times Z \times\{0\}},
$$

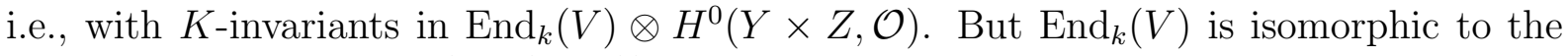
space of functions on $K$ (see $(1.1 .2))$, so

$$
\operatorname{End}\left(S_{Z, \beta}\right) \simeq H^{0}(K \times Y \times Z, \mathcal{O})^{K} \simeq H^{0}\left(K \times \pi_{0}(Z), \mathcal{O}\right)^{K} .
$$

The dimension of the latter space is $\left|\pi_{0}(Z)\right|$.

Example 2.4.6. Recall that in the case when $X$ is the standard ess-abelian variety $X_{A}=A \times \hat{A}$ and $Y=0 \times \hat{A}$ we have an equivalence $\mathcal{F}(Y, \mathcal{O}) \simeq D^{b}(A)$. Consider the 
Lagrangian subvariety $Z=L_{\phi} \subset A \times \hat{A}$ associated with a symmetric homomorphism $\phi \in$ $\operatorname{Hom}(A, \hat{A})_{\mathbb{Q}}^{+}$(see Example 2.2.4.1) and extend it in some way to a Lagrangian pair $(Z, \beta)$. Then by Theorem 2.4.5, the LI-sheaf $E=S_{Z, \beta} \in D^{b}(A)$ is an endosimple vector bundle. Furthermore, this bundle is semihomogeneous in the sense of [14], and $\phi$ corresponds to the element $\operatorname{det}(E) / \operatorname{rk}(E) \in N S(A)_{\mathbb{Q}}$ under the isomorphism (1.3.1) (see [14, Prop. 7.7]).

In the next proposition we study the relation between invariants of a generalized Lagrangian $j: Z \rightarrow X$ in $\mathcal{F}(Y, \alpha)$ and invariants of the corresponding Lagrangian $j\left(Z_{0}\right) \subset X$.

Proposition 2.4.7. Let $(Y, \alpha)$ be a Lagrangian pair.

(i) Let $(\bar{Z}, \bar{\beta})$ be a Lagrangian pair, transversal to $Y$, and let $j: Z_{0} \rightarrow \bar{Z}$ be a surjective homomorphism from an abelian variety $Z_{0}$ with finite kernel $C$, so that we have the generalized isotropic pair $\left(Z_{0}, \beta\right)$, where $\beta=j^{*} \bar{\beta}$. Consider the subgroup

$$
Z^{\prime}=\operatorname{ker}\left(X \stackrel{\eta}{\longrightarrow} \hat{X} \rightarrow \hat{Z}_{0}\right) \subset X .
$$

Then we have a natural duality

$$
\chi: Z^{\prime} / \bar{Z} \stackrel{\sim}{\longrightarrow} C^{*} .
$$

and an equivalence

$$
\Phi: \mathcal{F}(Y, \alpha)^{\left(Z_{0}, \beta\right)} \cap \operatorname{Coh}(X) \stackrel{\sim}{\longrightarrow} C-\text { rep }
$$

such that for $F \in \mathcal{F}(Y, \alpha)^{\left(Z_{0}, \beta\right)}$ and $z^{\prime} \in Z^{\prime}$ one has

$$
\Phi\left(T_{z^{\prime}} F\right) \simeq \chi\left(z^{\prime} \bmod \bar{Z}\right) \otimes \Phi(F) .
$$

Furthermore, $\Phi\left(S_{\bar{Z}, \bar{\beta}}\right)$ is isomorphic to the trivial one-dimensional representation of $C$.

(ii) Let $(j: Z \rightarrow X, \beta)$ be a generalized Lagrangian pair and let $Z_{0}$ be the connected component of zero in $Z$. Assume that the condition ( $\star$ from Theorem 2.4 .5 is satisfied. Consider the finite group scheme $\Pi=\pi_{0}(j(Z))=j(Z) / j\left(Z_{0}\right)$ as a subgroup of $X / j\left(Z_{0}\right)$. Assume first that $\Pi$ is reduced. Then there exists a $\Pi$-coset $\Pi^{\prime} \subset X / j\left(Z_{0}\right)$ such that

$$
S_{Z, \beta} \simeq\left(\bigoplus_{\bar{x} \in \Pi^{\prime}} T_{\bar{x}} S_{j\left(Z_{0}\right), \bar{\beta}}\right)^{\oplus N_{Z}}
$$

in $\mathcal{F}(Y, \alpha)$, where $\bar{\beta}$ is chosen in such a way that $\left(j\left(Z_{0}\right), \bar{\beta}\right)$ is a Lagrangian pair, and

$$
N_{Z}=\frac{\left|\pi_{0}(Z)\right|^{1 / 2}}{\left|\pi_{0}(j(Z))\right|^{1 / 2}}
$$

( $N_{Z}$ is always an integer). If $\Pi$ is not reduced then there is still a $\Pi$-coset $\Pi^{\prime} \subset X / j\left(Z_{0}\right)$ such that the composition factors of $S_{Z, \beta}$ in $\mathcal{F}(Y, \alpha)^{\left(Z_{0}, \beta\right)} \cap \operatorname{Coh}(X)$ (which is a finite length abelian category) are $\left(T_{\bar{x}} S_{j\left(Z_{0}\right), \bar{\beta}}\right), \bar{x} \in \Pi^{\prime}$, each with multiplicity $N_{Z} \cdot \frac{|\Pi|}{\# \Pi(k)}$.

Proof. (i) As in the proof of Theorem 2.4.5 the line bundle $L$ on $\left(Y \times Z_{0}\right) \times X$ given by (2.4.4) has a structure of a 1-cocycle for the (transitive) action of $Y \times Z_{0}$ on $X$. Furthermore, this 1-cocycle is a pull-back of a similar cocycle $\bar{L}$ on $(Y \times \bar{Z}) \times X$. Therefore, the central extension $G$ of $K=\operatorname{ker}\left(s_{Y, Z_{0}}\right)$ by $\mathbb{G}_{m}$ is the pull-back of the similar central 
extension $\bar{G}$ of $\bar{K}=\operatorname{ker}\left(s_{Y, \bar{Z}}\right)$ under the natural homomorphism $K \rightarrow \bar{K}$. Note that the exact sequence

$$
0 \rightarrow C \rightarrow Z_{0} \rightarrow \bar{Z} \rightarrow 0
$$

gives rise to an exact sequence

$$
0 \rightarrow C \rightarrow K \rightarrow \bar{K} \rightarrow 0
$$

Thus, we have an exact sequence of groups

$$
1 \rightarrow C \rightarrow G \rightarrow \bar{G} \rightarrow 1,
$$

where $C$ is a central subgroup in $G$ and $\bar{G}$ is a finite Heisenberg group. Thus, as in the proof of Theorem 2.4.5 we obtain an equivalence

$$
\mathcal{F}(Y, \alpha)^{\left(Z_{0}, \beta\right)} \cap \operatorname{Coh}(X) \simeq G-\operatorname{rep}_{1} .
$$

Since $\bar{G}$ is a Heisenberg group, the desired equivalence $\Phi$ follows from Lemma 1.2.1. Note that the natural functor

$$
\mathcal{F}(Y, \alpha)^{(\bar{Z}, \bar{\beta})} \rightarrow \mathcal{F}(Y, \alpha)^{Z_{0}, \beta}
$$

corresponds to the restriction under the homomorphism $G \rightarrow \bar{G}$. This implies that $\Phi\left(S_{\bar{Z}, \bar{\beta}}\right)$ corresponds to the trivial one-dimensional representation of $C$.

Now considering the dual of the exact sequence (2.4.8) we get (using the fact that $\bar{Z}$ is Lagrangian) the exact sequence

$$
C^{*} \rightarrow X / \bar{Z} \rightarrow \hat{Z}_{0} \rightarrow \ldots
$$

which gives an isomorphism of $Z^{\prime} / \bar{Z}$ with $C^{*}$. Thus, the pairing $\chi: Z^{\prime} / \bar{Z} \times C^{*} \rightarrow \mathbb{G}_{m}$ is obtained as the canonical pairing associated with the biextension $(\text { id } \times j)^{*} \overline{\mathcal{E}}$ of $X / \bar{Z} \times Z_{0}$, where $\overline{\mathcal{E}}$ is the biextension of $X / \bar{Z} \times \bar{Z}$ induced by $\mathcal{E}$. Explicitly, let

$$
t(C):\left.\mathcal{O}_{X / \bar{Z} \times C} \rightarrow(\mathrm{id} \times j)^{*} \overline{\mathcal{E}}\right|_{X / \bar{Z} \times C} \text { and } t\left(Z^{\prime} / \bar{Z}\right):\left.\mathcal{O}_{Z^{\prime} / \bar{Z} \times Z_{0}} \rightarrow(\mathrm{id} \times j)^{*} \overline{\mathcal{E}}\right|_{Z^{\prime} / \bar{Z} \times Z_{0}}
$$

be natural trivializations of restricted biextensions. Then

$$
\left.\chi \cdot t(C)\right|_{Z^{\prime} / \bar{Z} \times C}=\left.t\left(Z^{\prime} / \bar{Z}\right)\right|_{Z^{\prime} / \bar{Z} \times C} .
$$

The subgroup $Z^{\prime} \subset X$ acts on $X$ by translations, and we have an isomorphism of 1-cocycles of $Y \times Z_{0}$ with values in $\mathcal{P} i c(X)$

$$
L_{\left(y, z_{0}\right), x+z^{\prime}} \simeq L_{\left(y, z_{0}\right), x} \otimes \mathcal{B}_{z^{\prime}, j\left(z_{0}\right)}^{-1} \otimes \mathcal{B}_{y, z^{\prime}}^{-1}
$$

Using the trivialization of $\left.(\mathrm{id} \times j)^{*} \mathcal{E}\right|_{Z^{\prime} \times \bar{Z}}$ induced by $t\left(Z^{\prime} / \bar{Z}\right)$ we obtain an isomorphism

$$
\tau^{-1}: L_{\left(y, z_{0}\right), x+z^{\prime}} \stackrel{\sim}{\longrightarrow} L_{\left(y, z_{0}\right), x} \otimes \mathcal{B}_{j\left(z_{0}\right), z^{\prime}}^{-1} \otimes \mathcal{B}_{y, z^{\prime}}^{-1} \stackrel{\sim}{\longrightarrow} L_{\left(y, z_{0}\right), x} \otimes \mathcal{B}_{y+j\left(z_{0}\right), z^{\prime}}^{-1}
$$

Thus, for fixed $z^{\prime} \in Z^{\prime}$ we are in the situation of Proposition 1.2.2(iii) with $H=Y \times Z_{0}$, $f: X \rightarrow X$ the translation by $z^{\prime}, M=\left.\mathcal{B}\right|_{X \times\left\{z^{\prime}\right\}}$, the isomorphism $\tau_{f}=\tau$ given by $(2.4 .9)$ and the fixed point $x=0$. Note that the functor $T_{z^{\prime}}$ on $\mathcal{F}(Y, \alpha)$ sends $F$ to $t_{z^{\prime}}^{*} F \otimes M$, which is exactly the functor $\iota_{f, M}$ considered in Proposition 1.2.2(iii). Let $G_{z^{\prime}}$ be the 
central extension of $K$ by $\mathbb{G}_{m}$ with the underlying line bundle $\left.L\right|_{K \times\left\{z^{\prime}\right\}}$. The diagram (1.2.11) gives in our case an isomorphism of $G$-representations

$$
\operatorname{fib}_{0}\left(T_{z^{\prime}} F\right) \simeq \alpha^{*} \operatorname{fib}_{0}(F)
$$

for $F \in \mathcal{F}(Y, \alpha)^{\left(Z_{0}, \beta\right)}$, where the automorphism $\alpha=\kappa^{-1} \circ \bar{\tau}: G \rightarrow G$ (identical on $\mathbb{G}_{m} \subset G$ ) is the composition of isomorphisms of central extensions $\bar{\tau}: G \rightarrow G_{z^{\prime}}$ and $\kappa^{-1}: G_{z^{\prime}} \rightarrow G$ defined as follows. The isomorphism $\bar{\tau}: G \rightarrow G_{z^{\prime}}$ is obtained by specializing (2.4.9) to $\left(y, z_{0}\right) \in K$ and $x=0$, and using the trivialization of $\mathcal{B}_{0, z^{\prime}}$. On the other hand, choosing $\left(y^{\prime}, z_{0}^{\prime}\right)$ such that $y^{\prime}+j\left(z_{0}^{\prime}\right)=z^{\prime}$ we obtain another isomorphism $\kappa^{-1}: G_{z^{\prime}} \rightarrow G$ given by the isomorphism

$$
\begin{aligned}
& L_{\left(y, z_{0}\right), y^{\prime}+j\left(z_{0}^{\prime}\right)}=L_{\left(y, z_{0}\right), 0} \otimes \mathcal{B}_{y^{\prime}+j\left(z_{0}^{\prime}\right), j\left(z_{0}\right)}^{-1} \otimes \mathcal{B}_{y, y^{\prime}+j\left(z_{0}^{\prime}\right)}^{-1} \simeq \\
& L_{\left(y, z_{0}\right), 0} \otimes \mathcal{B}_{y^{\prime}, j\left(z_{0}\right)}^{-1} \otimes \mathcal{B}_{j\left(z_{0}\right), j\left(z_{0}^{\prime}\right)}^{-1} \otimes \mathcal{B}_{y^{\prime}, y}^{-1} \otimes \mathcal{B}_{y, j\left(z_{0}^{\prime}\right)}^{-1} \simeq \\
& L_{\left.\left(y, z_{0}\right), 0\right)} \otimes \mathcal{B}_{y^{\prime}, y+j\left(z_{0}\right)}^{-1} \otimes \mathcal{B}_{y+j\left(z_{0}\right), j\left(z_{0}^{\prime}\right)}^{-1} \simeq L_{\left.\left(y, z_{0}\right), 0\right)},
\end{aligned}
$$

where we used the symmetry of $\left.\mathcal{B}\right|_{Y \times Y}$ and of $\left.\mathcal{B}\right|_{\bar{Z} \times \bar{Z}}$ together with trivializations of $\mathcal{B}_{y^{\prime}, 0}$ and of $\mathcal{B}_{0, j\left(z_{0}^{\prime}\right)} \simeq \mathcal{B}_{j\left(z_{0}^{\prime}\right), 0}$. One can easily see from this that the restriction of $\alpha=\kappa^{-1} \circ \bar{\tau}$ to $C \times \mathbb{G}_{m} \subset G$ is given by $(c, \lambda) \mapsto\left(c, \chi_{z^{\prime}}(c) \lambda\right)$, where $\chi\left(z^{\prime}\right): C \rightarrow \mathbb{G}_{m}$ is the character corresponding to $z^{\prime} \bmod \bar{Z}$.

(ii) First, let us consider the case when $Y$ and $Z$ are transversal. Set $\bar{Z}=j\left(Z_{0}\right) \subset X$. By Lemma 2.2.5, $\bar{Z}$ is Lagrangian, so we can choose $\bar{\beta}$, so that $(\bar{Z}, \bar{\beta})$ is a Lagrangian pair. Now we have two completions of $Z_{0}$ to an isotropic pair: $\left(Z_{0},\left.\beta\right|_{Z_{0}}\right)$ and $\left(Z_{0}, j^{*} \bar{\beta}\right)$. Hence, $\left.\beta\right|_{Z_{0}} \simeq j^{*} \bar{\beta} \otimes \xi$ for some $\xi \in \hat{Z}_{0}$. Let us choose $x \in X$ such that $\xi_{z_{0}} \simeq \mathcal{E}_{j\left(z_{0}\right), x}$ and set

$$
\beta^{\prime}=\left.\beta \otimes \mathcal{E}^{-1}\right|_{Z \times\{x\}}
$$

Then $\left.\beta^{\prime}\right|_{Z_{0}} \simeq j^{*} \bar{\beta}$. On the other hand, by Lemma 2.4.2, we have an equivalence

$$
t(Z, x): \mathcal{F}(Y, \alpha)^{(Z, \beta)} \rightarrow \mathcal{F}(Y, \alpha)^{\left(Z, \beta^{\prime}\right)}
$$

induced by $T_{x}$. This equivalence sends $S_{Z, \beta}$ to $T_{x}\left(S_{Z, \beta}\right) \simeq S_{Z, \beta^{\prime}}$. Thus, it is enough to prove our statement with $\beta$ replaced by $\beta^{\prime}$. In other words, we can assume that $\left.\beta\right|_{Z_{0}} \simeq j^{*} \bar{\beta}$.

Let $G \rightarrow K=\operatorname{ker}\left(s_{Y, Z}\right)$ (resp., $G_{0} \rightarrow K_{0}=\operatorname{ker}\left(s_{Y, Z_{0}}\right)$ ) be the central extensions by $\mathbb{G}_{m}$ appearing in the proof of Theorem 2.4.5 (resp., in the proof of (i)), so that we have equivalences

$$
\mathcal{F}(Y, \alpha)^{(Z, \beta)} \cap \operatorname{Coh}(X) \simeq G-\operatorname{rep}_{1} \text { and } \mathcal{F}(Y, \alpha)^{\left(Z_{0}, \beta\right)} \cap \operatorname{Coh}(X) \simeq G_{0}-\operatorname{rep}_{1} .
$$

We have a commutative diagram of groups in which the horizontal arrows are injective

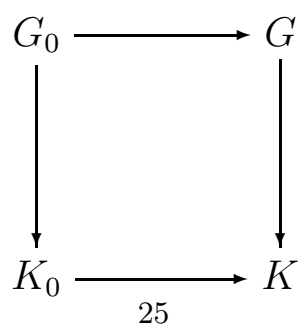


Under the equivalences (2.4.10) the natural functor

$$
\mathcal{F}(Y, \alpha)^{(Z, \beta)} \cap \operatorname{Coh}(X) \rightarrow \mathcal{F}(Y, \alpha)^{\left(Z_{0}, \beta\right)} \cap \operatorname{Coh}(X)
$$

corresponds to the restriction functor

$$
G-\mathrm{rep}_{1} \rightarrow G_{0}-\mathrm{rep}_{1} \simeq C-\mathrm{rep},
$$

where $C=\operatorname{ker}\left(\left.j\right|_{z_{0}}\right)$.

We claim that the subgroup $Z^{\prime} \subset X$ considered in (ii) coincides with $j(Z)$. Indeed, using the definition of $Z^{\prime}$ and the fact that $j: Z \rightarrow X$ is generalized Lagrangian we obtain a morphism of exact triangles

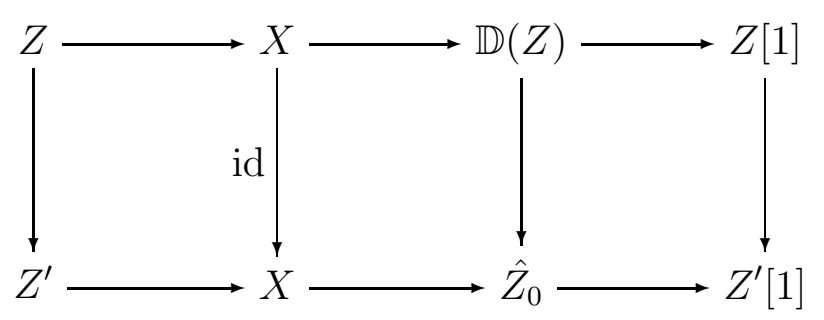

Since $H^{0} \mathbb{D}(Z) \simeq \hat{Z}_{0}$, this implies the surjectivity of the map $Z \rightarrow Z^{\prime}$, induced by $j$, which proves our claim.

Assume that $\Pi \simeq C^{*}$ is reduced. Then the restriction of the Schrödinger representation $V_{G}$ of $G$ to $G_{0}$ corresponds under the equivalence $G_{0}-$ rep $_{1} \simeq C$ - rep to a representation $\bigoplus_{\chi \in C^{*}} \chi^{\oplus m_{\chi}}$. Let

$$
V_{G}=\bigoplus_{\chi \in C^{*}}\left(V_{G}\right)_{\chi}
$$

be the decomposition of $V_{G}$, viewed as a representation of $C$, into isotypic components. It is well known that all $\left(V_{G}\right)_{\chi}$ have the same dimension (for example, this can be checked by embedding $C$ into a maximal isotropic subgroup of $K$ ), hence

$$
\operatorname{dim}\left(V_{G}\right)_{\chi}=\frac{\operatorname{dim} V_{G}}{|\Pi|} .
$$

Using Lemma 1.2.1, we see that

$$
m_{\chi} \operatorname{dim} V_{\bar{G}}=\operatorname{dim}\left(V_{G}\right)_{\chi},
$$

where $V_{\bar{G}}$ is the Schrödinger representation of $\bar{G}=G_{0} / C$. Thus, we obtain the following formula for the multiplicities:

$$
m_{\chi}=\frac{\operatorname{dim} V_{G}}{|\Pi| \cdot \operatorname{dim} V_{\bar{G}}} .
$$

Since $S_{\bar{Z}, \bar{\beta}}$ corresponds to the trivial representation of $C$, the isomorphism (2.4.7) will follow now from (2.4.6), once we show that $m_{\chi}=N_{Z}$, i.e.,

$$
\frac{\operatorname{dim} V_{G}}{\operatorname{dim} V_{\bar{G}}}=|\Pi|_{26}^{1 / 2} \cdot\left|\pi_{0}(Z)\right|^{1 / 2}
$$


(note that $\Pi=j(Z) / j\left(Z_{0}\right)=\pi_{0}(j(Z))$ ). Equivalently, we have to check that

$$
\frac{\operatorname{deg}\left(s_{Y, Z}\right)}{\operatorname{deg}\left(s_{Y, j\left(Z_{0}\right)}\right)}=|\Pi| \cdot\left|\pi_{0}(Z)\right| .
$$

Consider the commutative diagram of isogenies

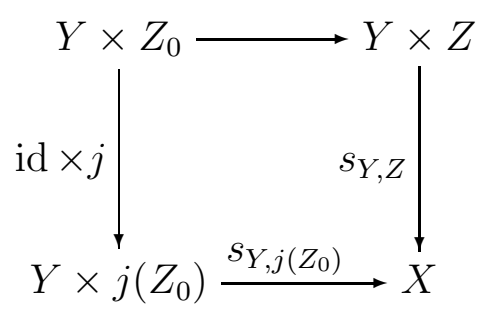

where the top horizontal arrow is an embedding of the connected component of zero. Note that

$$
\operatorname{deg}\left(j: Z_{0} \rightarrow j\left(Z_{0}\right)\right)=|C|=|\Pi|
$$

due to duality between $\Pi$ and $C$. Hence, this diagram leads to the following equality of degrees

$$
\operatorname{deg}\left(s_{Y, Z_{0}}\right)=\frac{\operatorname{deg}\left(s_{Y, Z}\right)}{\left|\pi_{0}(Z)\right|}=|\Pi| \cdot \operatorname{deg}\left(s_{Y, j\left(Z_{0}\right)}\right)
$$

which gives the desired identity.

In the case when $\Pi$ is not reduced the representation of $C$ corresponding to $\left.V_{G}\right|_{G_{0}}$ has a composition series, where the multiplicity $m_{\chi}$ of a character $\chi \in C^{*}(k)$ is given by a formula similar to (2.4.11) but with $|\Pi|$ replaced by $\# \Pi(k)$. This leads to the factor $\frac{|\Pi|}{\# \Pi(k)}$ in the multiplicities of the composition series of $S_{Z, \beta}$ in $\mathcal{F}(Y, \alpha)^{\left(Z_{0}, \beta\right)} \cap \operatorname{Coh}(X)$.

Now let us consider the case when $Y$ and $Z$ are not necessarily transversal. As in the proof of Theorem 2.4.5, using Lemma 2.2.7 we choose a Lagrangian pair $\left(Y^{\prime}, \alpha^{\prime}\right)$ transversal to $Z$, such that we have an equivalence $\psi: \mathcal{F}(Y, \alpha)_{S} \simeq \mathcal{F}\left(Y^{\prime}, \alpha^{\prime}\right)_{S}$ compatible with $\mathbf{H}$-action. Then $\psi$ induces an equivalence

$$
\mathcal{F}(Y, \alpha)^{\left(Z_{0}, \beta\right)} \simeq \mathcal{F}\left(Y^{\prime}, \alpha^{\prime}\right)^{\left(Z_{0}, \beta\right)}
$$

(and similar equivalences for $(Z, \beta)$-invariants and for $\left(j\left(Z_{0}\right), \bar{\beta}\right)$-invariants). As we have seen in the proof of Theorem 2.4.5, $\psi\left(S_{j\left(Z_{0}\right), \bar{\beta}}(Y, \alpha)\right)$ is cohomologically pure, so changing $\psi$ by $\psi[m]$ for appropriate $m \in \mathbb{Z}$ we can assume that $\psi$ induces an equivalence

$$
\mathcal{F}(Y, \alpha)^{\left(j\left(Z_{0}\right), \bar{\beta}\right)} \cap \operatorname{Coh}(X) \simeq \mathcal{F}\left(Y^{\prime}, \alpha^{\prime}\right)^{\left(j\left(Z_{0}\right), \bar{\beta}\right)} \cap \operatorname{Coh}(X) .
$$

We claim that in this case $\psi$ also induces an equivalence

$$
\mathcal{F}(Y, \alpha)^{\left(Z_{0}, \beta\right)} \cap \operatorname{Coh}(X) \simeq \mathcal{F}\left(Y^{\prime}, \alpha^{\prime}\right)^{\left(Z_{0}, \beta\right)} \cap \operatorname{Coh}(X) .
$$

Indeed, we know that all simple objects of the finite length abelian category $\mathcal{F}\left(Y^{\prime}, \alpha^{\prime}\right)^{\left(Z_{0}, \beta\right)} \cap$ $\operatorname{Coh}(X)$ are of the form $T_{x} S$, where $S=S_{j\left(Z_{0}\right), \bar{\beta}}\left(Y^{\prime}, \alpha^{\prime}\right)$. But $\psi^{-1}\left(T_{x} S\right)=T_{x} \psi^{-1}(S)$ is a coherent sheaf. Hence, $\psi^{-1}$ sends $\mathcal{F}\left(Y^{\prime}, \alpha^{\prime}\right)^{\left(Z_{0}, \beta\right)} \cap \operatorname{Coh}(X)$ to coherent sheaves. Since the subcategory $\mathcal{F}\left(Y^{\prime}, \alpha^{\prime}\right)^{\left(Z_{0}, \beta\right)} \subset D^{b}(X)$ is compatible with the devissage with respect to the standard $t$-structure, this easily implies that $\psi$ sends $\mathcal{F}(Y, \alpha)^{\left(Z_{0}, \beta\right)} \cap \operatorname{Coh}(X)$ to coherent 
sheaves and our claim follows. Using the equivalence (2.4.13), we can transfer the decomposition of $S_{Z, \beta}\left(Y^{\prime}, \alpha^{\prime}\right)$ in the category $\mathcal{F}\left(Y^{\prime}, \alpha^{\prime}\right)^{\left(Z_{0}, \beta\right)} \cap \operatorname{Coh}(X)$ to that of $S_{Z, \beta}(Y, \alpha)$ in the category $\mathcal{F}(Y, \alpha)^{\left(Z_{0}, \beta\right)} \cap \operatorname{Coh}(X)$.

Corollary 2.4.8. Let $(j: Z \rightarrow X, \beta)$ be a generalized Lagrangian pair. Assume that $\operatorname{char}(k)=0$. Then

$$
S_{Z, \beta} \simeq \bigoplus_{i=1}^{N} S_{j\left(Z_{0}\right), \beta_{i}}
$$

for some line bundles $\beta_{1}, \ldots, \beta_{N}$ on $j\left(Z_{0}\right)$ such that $\left(j\left(Z_{0}\right), \beta_{i}\right)$ are Lagrangian pairs.

Proof. This follows from Proposition 2.4.7(ii) together with Lemma 2.4.2.

Definition 2.4.9. Let $X=X_{A}$ and $Y=\{0\} \times \hat{A}$, so that we have $\mathcal{F}(Y, \mathcal{O}) \simeq D^{b}(A)$ (see Example 2.3.2). A coherent sheaf $F$ on $A$ is called a $g L I$-sheaf if there exists a generalized Lagrangian pair $(Z, \beta)$ such that $F$ is $(Z, \beta)$-invariant. We say that $F$ is an $L I$-sheaf if $Z$ can be chosen to be a subvariety in $X_{A}$.

We can show that for an LI-sheaf the subvariety $Z$ in the above definition can be recovered as the stabilizer with respect to the action of $\mathbf{H}$. Here is a slightly more general result.

Proposition 2.4.10. Let $(Y, \alpha)$ and $(Z, \beta)$ be Lagrangian pairs for an ess-abelian variety $X$. Assume that the condition $(\star)$ from Theorem 2.4.5 is satisfied and consider the generating object $S_{Z, \beta}=S_{Z, \beta}(Y, \alpha) \in \mathcal{F}(Y, \alpha)^{(Z, \beta)} \cap \operatorname{Coh}(X)$. Then the subset of points $x \in X$ such that $T_{x}\left(S_{Z, \beta}\right) \simeq S_{Z, \beta}$ coincides with $Z$.

Proof. The same argument as in the proof of Theorem 2.4.5 (based on Lemma 2.2.7 and Theorem 2.3.4) shows that it is enough to consider the case when $Y$ and $Z$ are transversal. In this case $S_{Z, \beta}$ is a vector bundle (see Theorem 2.4.5). Suppose $T_{x}\left(S_{Z, \beta}\right) \simeq S_{Z, \beta}$ for some $x \in X$. By definition of $(Z, \beta)$-invariance, we have an isomorphism

$$
\beta_{z} \otimes T_{z}\left(p^{*} S_{Z, \beta}\right) \simeq p^{*} S_{Z, \beta}
$$

in $\mathcal{F}(Y, \alpha)_{Z} \subset D^{b}(X \times Z)$, where $z=z^{u n} \in Z(Z)$ is the universal point and $p: X \times Z \rightarrow X$ is the projection. This leads to isomorphisms

$$
\beta_{z} \otimes T_{z} T_{x}\left(p^{*} S_{Z, \beta}\right) \simeq \beta_{z} \otimes T_{x} T_{z}\left(p^{*} S_{Z, \beta}\right) \simeq p^{*} S_{Z, \beta}
$$

in $\mathcal{F}(Y, \alpha)_{Z}$. Since the commutator in the Heisenberg groupoid is given by the biextension $\mathcal{E}$, we deduce an isomorphism

$$
\mathcal{E}_{z, x} \otimes p^{*} S_{Z, \beta} \simeq p^{*} S_{Z, \beta}
$$

on $Z \times X$. Restricting to $Z \times\{0\}$ we get a trivialization of the line bundle $\mathcal{E}_{z, x}$ on $Z$. Since $Z$ is Lagrangian, this implies that $x \in Z$.

Corollary 2.4.11. Keep the assumptions of Proposition 2.4.10. Suppose that $S_{Z, \beta}$ is invariant with respect to another Lagrangian pair $\left(Z^{\prime}, \beta^{\prime}\right)$. Then $Z^{\prime}=Z$ and $\beta^{\prime}=\beta$. 
Proof. Proposition 2.4.10 implies that $Z^{\prime} \subset Z$, hence $Z^{\prime}=Z$ (since $\operatorname{dim} Z=\operatorname{dim} Z^{\prime}$ ). By Lemma 2.2.7, we can assume that $Y$ is transversal to $Z$, so that $S_{Z, \beta} \in \mathcal{F}(Y, \alpha)$ is a vector bundle (see Theorem 2.4.5). Now the isomorphism

$$
\beta_{z} \otimes T_{z}\left(S_{Z, \beta}\right) \simeq \beta_{z}^{\prime} \otimes T_{z}\left(S_{Z, \beta}\right)
$$

on $Z \times X$ leads to

$$
\beta_{z}^{-1} \otimes \beta_{z}^{\prime} \otimes S_{Z, \beta} \simeq S_{Z, \beta}
$$

Restricting to $Z \times\{0\}$ we deduce the triviality of $\beta^{-1} \otimes \beta^{\prime}$.

The formula for $S_{Z, \beta}(Y, \alpha)$ from Theorem 2.4.5 has the following analog in the nontransversal case.

Proposition 2.4.12. Let $(Y, \alpha)$ be a Lagrangian pair, and let $(j: Z \rightarrow X, \beta)$ be a generalized Lagrangian pair. Assume that the condition ( $\star$ ) from Theorem 2.4.5 holds. Assume in addition that the pull-backs of $\alpha$ and $\beta$ to the connected component of zero in $Y \times_{X} Z$ are isomorphic. Then $S_{Z, \beta}(Y, \alpha)$ is a direct summand in

$$
\left(s_{Y, Z}\right)_{*}\left(\alpha^{-1} \otimes \beta^{-1} \otimes(i \times j)^{*} \mathcal{B}^{-1}\right),
$$

where $i: Y \rightarrow X$ is the embedding map.

Proof. We can factor the homomorphism $s_{Y, Z}: Y \times Z \rightarrow X$ as a composition $Y \times$

$Z \stackrel{q}{\longrightarrow} X^{\prime} \stackrel{\iota}{\longrightarrow} X$, where $q$ is surjective and $\iota$ is injective. Now as in the proof of Theorem 2.4.5, we obtain a structure of 1-cocycle with respect to the action of $Y \times Z$ on $X^{\prime}$ on the line bundle $L$ on $(Y \times Z) \times X^{\prime}$ given by the restriction of (2.4.4). Furthermore, we see that for an $L$-twisted $Y \times Z$-equivariant sheaf $F$ on $X^{\prime}$ one has $\iota_{*} F \in \mathcal{F}(Y, \alpha)^{(Z, \beta)}$. The coherent sheaf

$$
S=q_{*}\left(\left.L\right|_{Y \times Z \times\{0\}}\right)=q_{*}\left(\alpha^{-1} \otimes \beta^{-1} \otimes(i \times j)^{*} \mathcal{B}^{-1}\right)
$$

on $X^{\prime}$ has a natural $L$-twisted $Y \times Z$-equivariant structure coming from the structure of a 1-cocycle on $L$. Thus, by Theorem 2.4.5, $S_{Z, \beta}(Y, \alpha)$ is a direct summand of $S$ provided $S \neq 0$.

Now we observe that for $y+j(z)=0$, where $y \in Y, z \in Z$, we have an isomorphism

$$
\mathcal{B}_{y, j(z)}^{-1} \simeq \alpha_{0}^{-1} \otimes \alpha_{y} \otimes \alpha_{-y} .
$$

Hence, we obtain the isomorphism of the restriction of $L$ to $\operatorname{ker}\left(s_{Y, Z}\right) \times\{0\}$ with $\alpha_{-y} \otimes$ $\beta_{z}^{-1}$. Under the isomorphism $Y \times_{X} Z \rightarrow \operatorname{ker}\left(s_{Y, Z}\right):(y, z) \mapsto(-y, z)$ this line bundle corresponds to the difference between the restrictions of $\alpha$ and $\beta$. Thus, if $A$ is the connected component of zero in $\operatorname{ker}\left(s_{Y, Z}\right)$, then our assumption on $\alpha$ and $\beta$ implies that $\left.L\right|_{A \times\{0\}} \simeq \mathcal{O}$, hence $S \neq 0$.

\section{Functors associated With Lagrangian CORRESPONDENCES}

\subsection{Lagrangian correspondences for ess-abelian varieties.}

Definition 3.1.1. Let $\left(X, \mathcal{B}_{X}\right)$ and $\left(Y, \mathcal{B}_{Y}\right)$ be ess-abelian varieties. A generalized Lagrangian correspondence ( $g$-Lag-correspondence for short) from $X$ to $Y$ is a generalized Lagrangian pair $(L, \alpha)$ for $\left(X \times Y, \mathcal{B}_{X}^{-1} \otimes \mathcal{B}_{Y}\right)$. In the case when $L$ is a subvariety of $X \times Y$ 
(i.e., $(L, \alpha)$ is a Lagrangian pair), we say that we have a Lagrangian correspondence. Note that with every g-Lag-correspondence $(L, \alpha)$ from $X$ to $Y$ one can associate the opposite $g$-Lag-correspondence $\left(\sigma(L), \alpha^{-1}\right)$ from $Y$ to $X$, where $\sigma: X \times Y \rightarrow Y \times X$ is a natural isomorphism.

Example 3.1.2. Let $f \in \operatorname{Hom}(X, Y)_{\mathbb{Q}}$ be an isomorphism between $X$ and $Y$ in the category $\mathcal{A} b_{\mathbb{Q}}$, so that we have an equality

$$
\eta_{X}=\hat{f} \circ \eta_{Y} \circ f
$$

where $\eta_{X}: X \rightarrow \hat{X}$ and $\eta_{Y}: Y \rightarrow \hat{Y}$ are the symplectic self-dualities (in this situation we say that $f$ is symplectic). Then similarly to Example 2.2.4.1, we consider the graph of $f$

$$
L(f)=\{(N x, N f(x)) \mid x \in X\} \subset X \times Y,
$$

where $N>0$ is an integer such that $N f \in \operatorname{Hom}(X, Y)$. The equality (3.1.1) implies an isomorphism

$$
(N f \times N f)^{*} \mathcal{E}_{Y} \simeq \mathcal{E}_{X}^{N^{2}} \simeq\left(N \operatorname{id}_{X} \times N \operatorname{id}_{X}\right)^{*} \mathcal{E}_{X}
$$

of biextensions of $X \times X$. Let $p_{X}: L(f) \rightarrow X, p_{Y}: L(f) \rightarrow Y$ and $\pi: X \rightarrow L(f)$ be the natural maps. Then the previous isomorphism can be rewritten as

$$
(\pi \times \pi)^{*}\left(p_{Y} \times p_{Y}\right)^{*} \mathcal{E}_{Y} \simeq(\pi \times \pi)^{*}\left(p_{X} \times p_{X}\right)^{*} \mathcal{E}_{X}
$$

Hence, $\left(p_{Y} \times p_{Y}\right)^{*} \mathcal{E}_{Y} \simeq\left(p_{X} \times p_{X}\right)^{*} \mathcal{E}_{X}$, i.e., $L(f)$ is isotropic. Since $\operatorname{dim} L(f)=\operatorname{dim} X=$ $\operatorname{dim}(X \times Y) / 2$, by Lemma 2.2.3(ii), it is Lagrangian. Conversely, it is easy to see that all Lagrangian correspondences $L \subset X \times Y$, finite over $X$, are obtained by the above construction (in particular, they are automatically finite over $Y$ ).

If $(L, \alpha)$ is a g-Lag-correspondence from $X$ to $Y$ and $(M, \beta)$ is a g-Lag-correspondence from $Y$ to $Z$ then we can try to define the composition $(M \circ L, \beta \circ \alpha)$ by setting $M \circ L=$ $L \times_{Y} M$ and defining $\beta \circ \alpha$ as the tensor product of the pull-backs of $\alpha$ and $\beta$ to $L \times_{Y} M$. Below we will give a sufficient condition for $(M \circ L, \beta \circ \alpha)$ to be a g-Lag-correspondence from $X$ to $Z$ (see Corollary 3.1.4).

It is convenient to consider the following more general setup. Let $(X, \eta, \mathcal{B})$ be an ess-abelian variety. Assume that $I \subset X$ is an isotropic abelian subvariety so that the composition $I \rightarrow X \stackrel{\eta}{\rightarrow} \hat{X} \rightarrow \hat{I}$ is zero. In other words, we have $I \subset I^{\perp}$, where $I^{\perp}$ is the kernel of the composition $X \rightarrow \hat{X} \rightarrow \hat{I}$. Then we can define the reduced ss-abelian variety $(\bar{X}, \bar{\eta})$, where $\bar{X}=I^{\perp} / I$ and $\bar{\eta}$ is induced by $\eta$. Moreover, if we assume that the restriction $\left.\mathcal{B}\right|_{I \times I^{\perp}}$ is trivial then there is an induced biextension $\overline{\mathcal{B}}$ on $\bar{X} \times \bar{X}$, so that $(\bar{X}, \overline{\mathcal{B}})$ is an ess-abelian variety.

In this situation one can start with a generalized Lagrangian pair $(Y, \alpha)$ for $X$ and try to define the corresponding Lagrangian pair for $\bar{X}$.

Proposition 3.1.3. Let $(Y, \alpha)$ be a generalized Lagrangian pair for $X$ such that the natural map $Y \rightarrow X / I^{\perp}$ is surjective. Set $\bar{Y}=Y \times_{X} I^{\perp}$ and let $\bar{\alpha}$ be the pull-back of $\alpha$ to $\bar{Y}$. Then $(\bar{Y}, \bar{\alpha})$ is a generalized Lagrangian pair for $\bar{X}$.

Proof. By assumption we have an exact sequence of commutative groups

$$
0 \rightarrow \bar{Y} \rightarrow Y \rightarrow X / I^{\perp} \rightarrow 0
$$


where the map $Y \rightarrow X / I^{\perp}$ is the composition of the natural maps $Y \rightarrow X$ and $X \rightarrow X / I^{\perp}$. Dualizing and using the fact that $(Y, \alpha)$ is generalized Largangian we obtain an exact triangle in $D^{b}\left(\mathcal{G}^{p r}\right)$

$$
I \rightarrow[Y \rightarrow X] \rightarrow \mathbb{D}(\bar{Y}) \rightarrow \ldots
$$

where the first arrow is the composition $I \rightarrow X \rightarrow[Y \rightarrow X]$. By the octahedron axiom we obtain also an exact triangle

$$
Y \rightarrow X / I \rightarrow \mathbb{D}(\bar{Y}) \rightarrow \ldots
$$

In other words, $\mathbb{D}(\bar{Y})$ is represented by the complex $[Y \rightarrow X / I]$. It follows that the map $Y \rightarrow X / I$ has finite kernel. Now the cartesian square

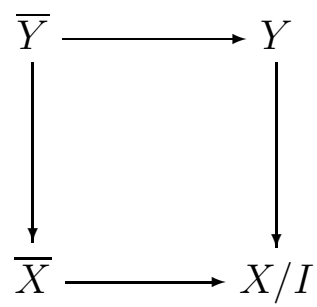

leads to the exact triangle

$$
\bar{Y} \rightarrow \bar{X} \rightarrow \mathbb{D}(\bar{Y}) \rightarrow \ldots
$$

It is easy to deduce from this that the pair $(\bar{Y}, \bar{\alpha})$ is generalized Lagrangian.

Corollary 3.1.4. Let $(L, \alpha)$ (resp., $(M, \beta)$ ) be a g-Lag-correspondence from $X$ to $Y$ (resp., from $Y$ to $Z$ ). Assume that the natural map $L \times M \rightarrow Y$ is surjective. Then $(M \circ L, \beta \circ \alpha)$ is a $g$-Lag-correspondence from $X$ to $Z$.

Proof. Consider the ess-abelian variety

$$
\left(X \times Y \times Y \times Z, \mathcal{B}_{X}^{-1} \otimes \mathcal{B}_{Y} \otimes \mathcal{B}_{Y}^{-1} \otimes \mathcal{B}_{Z}\right)
$$

and the isotropic subvariety $I=0_{X} \times \Delta(Y) \times 0_{Z}$, where $\Delta: Y \rightarrow Y \times Y$ is the diagonal embedding. To get the result we apply Proposition 3.1.3 to the generalized Lagrangian pair $(L \times M, \alpha \otimes \beta)$ for this ess-abelian variety.

This result allows us to make the following

Definition 3.1.5. (i) For an ess-abelian variety $\left(X, \mathcal{B}_{X}\right)$ we denote by $\operatorname{Lag}(X)$ the set of isomorphism classes of g-Lag-correspondences $(L, \alpha)$ from $X$ to $X$ such that the projections $p_{1}, p_{2}: L \rightarrow X$ are surjective. The composition of correspondences makes $\operatorname{Lag}(X)$ into a monoid, where the unit corresponds to $L=\Delta(X) \subset X \times X$ (and trivial $\alpha$ ).

(ii) For an ss-abelian variety $X$ we denote by $U(X, \mathbb{Q})$ the group of symplectic automorphisms of $X$ in $\mathcal{A} b_{\mathbb{Q}}$ (see Example 3.1.2).

Lemma 3.1.6. Associating with $(i: L \rightarrow X, \alpha) \in \operatorname{Lag}(X)$ the morphism in $\operatorname{End}(X)_{\mathbb{Q}}$ given by the correspondence $i\left(L_{0}\right) \subset X \times X$ gives a surjective homomorphism of monoids

$$
\pi: \operatorname{Lag}(X) \rightarrow U(X, \mathbb{Q}) .
$$


Proof. Since the projection $p_{1} \circ i: L_{0} \rightarrow X$ is an isogeny there exists a morphism $\phi: X \rightarrow L_{0}$ such that

$$
i(\phi(x))=(n x, a(x))
$$

for some integer $n>0$ and some element $a \in \operatorname{End}(X)$. By definition, $\pi(L)=a / n \in$ $\operatorname{End}(X)_{\mathbb{Q}}$. Now let $(j: M \rightarrow X, \beta)$ be another element of $\operatorname{Lag}(X)$, and let $\psi: X \rightarrow M_{0}$ be a morphism such that

$$
j(\psi(x))=(m x, b(x))
$$

for some integer $m>0$ and some element $b \in \operatorname{End}(X)$, and so $\pi(M)=b / m$. Then we have a morphism

$$
X \rightarrow M \circ L=L \times_{X} M: x \mapsto(\operatorname{mnx}, \operatorname{ma}(x), b a(x))
$$

which factors through the connected component of zero in $M \circ L$. Thus,

$$
\pi(M \circ L)=\frac{b a}{m n}=\pi(M) \circ \pi(L) .
$$

The homomorphism $\pi$ is surjective since the map $g \mapsto L(g)$ gives its set-theoretic section (see Example 3.1.2).

Later we will need the following simple result about the composition of correspondences. For a g-Lag-correspondence $L \rightarrow X \times Y$ let us set

$$
q(L)=\operatorname{deg}(L \rightarrow X)
$$

with the convention that this is zero if the projection $L \rightarrow X$ is not an isogeny.

Lemma 3.1.7. For $g$-Lag-correspondence $L \rightarrow X \times Y$ and $M \rightarrow Y \times Z$ such that the map $L \times M \rightarrow Y$ is surjective one has

$$
q(M \circ L)=q(L) \cdot q(M)
$$

Proof. The projection $L \times_{Y} M \rightarrow X$ factors as the composition $L \times_{Y} M \rightarrow L \rightarrow X$, so

$$
q(M \circ L)=q(L) \cdot \operatorname{deg}\left(L \times_{Y} M \rightarrow L\right) .
$$

Now the cartesian square

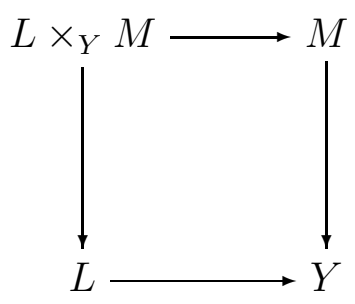

shows that $\operatorname{deg}\left(L \times_{Y} M \rightarrow L\right)=\operatorname{deg}(M \rightarrow Y)=q(M)$. 
3.2. LI-kernels and functors. Recall that with every abelian variety $A$ we associate the standard ess-abelian variety $X_{A}=A \times \hat{A}$ equipped with a symplectic biextension $\mathcal{E}_{A}=\mathcal{B}_{A} \otimes \sigma^{*} \mathcal{B}_{A}^{-1}$, where $\mathcal{B}_{A}=p_{14}^{*} \mathcal{P}$. The corresponding Heisenberg groupoid acts on the category $D^{b}(A)$ (see Example (2.3.2)).

Definition 3.2.1. (i) Let $A$ and $B$ be abelian varieties. Given a g-Lag-correspondence $(L, \alpha)$ from $X_{A}$ to $X_{B}$ we can consider the setup of Theorem 2.4.5 for the Heisenberg groupoid associated with $\left(X_{A} \times X_{B}, \mathcal{B}_{A}^{-1} \otimes \mathcal{B}_{B}\right)$, acting on the category $D^{b}(A \times B)$ that can be identified with $\mathcal{F}(Y, \alpha)$ for $Y=\{0\} \times \hat{A} \times\{0\} \times \hat{B} \subset X_{A} \times X_{B}$ and $\alpha=\mathcal{O}$ (see Remark 2.3.1.1). Therefore, we have the $t$-generator $S_{L, \alpha} \in \operatorname{Coh}(A \times B)$ of $(L, \alpha)$-invariant objects in $D^{b}(A \times B)$ (see Theorem 2.4.5). We call $S_{L, \alpha}$ the gLI-kernel defined by $(L, \alpha)$ and denote the corresponding gLI-functor by

$$
\Phi_{L, \alpha}:=\Phi_{S_{L, \alpha}}: D^{b}(A) \rightarrow D^{b}(B) .
$$

In the case when $L$ is a subvariety of $X_{A} \times X_{B}$ we call $S_{L, \alpha}$ (resp., $\Phi_{L, \alpha}$ ) the LI-kernel (resp., LI-functor) defined by $(L, \alpha)$

(ii) We say that a g-Lag-correspondence $L \rightarrow X_{A} \times X_{B}=A \times \hat{A} \times B \times \hat{B}$ is nondegenerate if the projection $p_{A B}: L \rightarrow A \times B$ is surjective.

For a g-Lag-correspondence $L \rightarrow X_{A} \times X_{B}$ we will denote projections to products of factors in $X_{A} \times X_{B}$ as $p_{A}: L \rightarrow A, p_{A \hat{A}}: L \rightarrow A \times \hat{A}$, etc.

Example 3.2.2. Let $L(g)$ be the Lagrangian correspondence from $X_{A}$ to itself associated with an element $g \in U\left(X_{A}, \mathbb{Q}\right)$ (see Example 3.1.2). Let us write

$g=\left(\begin{array}{ll}a & b \\ c & d\end{array}\right)$, where $a \in \operatorname{Hom}(A, A)_{\mathbb{Q}}, b \in \operatorname{Hom}(\hat{A}, A)_{\mathbb{Q}}, c \in \operatorname{Hom}(A, \hat{A})_{\mathbb{Q}}, d \in \operatorname{Hom}(\hat{A}, \hat{A})_{\mathbb{Q}}$.

Then the correspondence $L(g)$ is nondegenerate if and only if the map in $\mathcal{A} b_{\mathbb{Q}}$

$$
A \times \hat{A} \rightarrow A \times A:(x, \xi) \mapsto(x, a x+b \xi)
$$

is an isomorphism. Equivalently, $b$ should be invertible in $\mathcal{A} b_{\mathbb{Q}}$.

The proof of the following lemma is straightforward and is left to the reader.

Lemma 3.2.3. Under the natural identification of $D^{b}(A \times B)$ with $\mathcal{F}(Y, \mathcal{O})$, where $Y=$ $\{0\} \times \hat{A} \times\{0\} \times \hat{B} \subset X_{A} \times X_{B}$ one has for $K \in D^{b}(A \times B)$

$$
T_{(a, \xi, b, \eta)}(K)=t_{(a, b)}^{*} K \otimes\left(\mathcal{P}_{-\xi} \otimes \mathcal{P}_{\eta}\right)=\left(\mathbb{T}_{a, \xi}^{A}\right)^{-1} \circ_{A} K \circ_{B} \mathbb{T}_{b, \eta}^{B},
$$

where $a \in A, \xi \in \hat{A}, b \in B, \eta \in \hat{B}$. Here $\mathbb{T}_{x}^{A}$ are the kernels (2.3.2) and $\left(\mathbb{T}_{a, \xi}^{A}\right)^{-1}=$ $\mathcal{P}_{a, \xi} \otimes \mathbb{T}_{-a,-\xi}^{A}$.

This lemma allows to rewrite the condition of $(L, \alpha)$-invariance for $K \in D^{b}(A \times B)$ as an isomorphism

$$
K \simeq \alpha_{l} \otimes\left(\left(\mathbb{T}_{p_{A \hat{A}}(l)}^{A}\right)^{-1} \circ_{A} K \circ_{B} \mathbb{T}_{p_{B \hat{B}}(l)}^{B}\right),
$$

for $l \in L$, or equivalently,

$$
\mathbb{T}_{p_{A \hat{A}}(l)}^{A} \circ_{A} K \simeq \alpha_{l} \otimes K \circ_{B} \mathbb{T}_{p_{B \hat{B}}(l)}^{B} .
$$


Hence, the gLI-functor associated with $(L, \alpha)$ satisfies the following "intertwining" isomorphisms involving Heisenberg groupoids actions on $D^{b}(A)$ and $D^{b}(B)$ :

$$
\Phi_{L, \alpha} \circ T_{p_{A \hat{A}}(l)} \simeq \alpha_{l} \otimes T_{p_{B \hat{B}}(l)} \circ \Phi_{L, \alpha}
$$

for $l \in L$.

In the case when both projections $L \rightarrow X_{A}$ and $L \rightarrow X_{B}$ are surjective, i.e., $(L, \alpha)$ is an element of $\operatorname{Lag}\left(X_{A}\right)$ (see Definition 3.1.5(i)), we can use (3.2.1) to move the kernels of the form $\mathbb{T}_{x}^{A}$ through $K$. We record this observation for future use in the next lemma.

Lemma 3.2.4. Let $(L, \alpha)$ be a g-Lag-correspondence in $\operatorname{Lag}\left(X_{A}\right)$ and let $K \in D^{b}(A \times A)$ be an $(L, \alpha)$-invariant kernel. For any $x \in X_{A}$ there exists $x^{\prime} \in X_{A}$ and $x^{\prime \prime} \in X_{A}$ such that

in $D^{b}(A \times A)$.

$$
K \circ_{A} \mathbb{T}_{x}^{A}=\mathbb{T}_{x^{\prime}}^{A} \circ_{A} K \text { and } \mathbb{T}_{x}^{A} \circ_{A} K=K \circ_{A} \mathbb{T}_{x^{\prime \prime}}^{A}
$$

From Theorem 2.4.5 and Proposition 2.4.12 we get the following (almost) explicit formulas for $S_{L, \alpha}$.

Lemma 3.2.5. (i) If $(L, \alpha)$ is a nondegenerate $g$-Lag-correspondence from $X_{A}$ to $X_{B}$ then $S_{L, \alpha}$ is a vector bundle on $A \times B$ given by

$$
S_{L, \alpha} \simeq p_{A B *}\left(\alpha^{-1} \otimes p_{A \hat{A}}^{*} \mathcal{P}^{-1} \otimes p_{B \hat{B}}^{*} \mathcal{P}\right)^{I},
$$

where $p_{A \hat{A}}$ and $p_{B \hat{B}}$ are projections from $L$ to $X_{A}=A \times \hat{A}$ and $X_{B}=B \times \hat{B}$, respectively; $I$ is a Lagrangian subgroup in $G:=\operatorname{ker}\left(p_{A B}\right)$ with respect to the central extension of $G$ associated with $\left.\alpha\right|_{G}$.

(ii) Let $(L, \alpha)$ be an arbitrary $g$-Lag-correspondence from $X_{A}$ to $X_{B}$. Assume that the restriction of $\alpha$ to the connected component of zero in $\operatorname{ker}\left(p_{A B}\right)$ is trivial. Then $S_{L, \alpha} \in$ $\operatorname{Coh}(A \times B)$ is a direct summand in

$$
p_{A B *}\left(\alpha^{-1} \otimes p_{A \hat{A}}^{*} \mathcal{P}^{-1} \otimes p_{B \hat{B}}^{*} \mathcal{P}\right) .
$$

Proof. (i) Applying Theorem 2.4.5 to the generalized Lagrangians $Z=L \rightarrow X_{A} \times X_{B}$ and $Y=\{0\} \times \hat{A} \times\{0\} \times \hat{B} \subset X_{A} \times X_{B}$, we obtain that $S_{L, \alpha}$ as an object of $\mathcal{F}(Y, \mathcal{O})$ is given by

$$
S_{L, \alpha} \simeq q_{*}\left(\alpha(l)^{-1} \otimes \mathcal{P}_{p_{A}(l), \xi} \otimes \mathcal{P}_{p_{B}(l), \eta}^{-1}\right)^{I},
$$

where $(l, \xi, \eta) \in L \times \hat{A} \times \hat{B}$, and $q$ is the map

$$
L \times \hat{A} \times \hat{B} \rightarrow A \times \hat{A} \times B \times \hat{B}:(l, \xi, \eta) \mapsto\left(p_{A}(l), p_{\hat{A}}(l)+\xi, p_{B}(l), p_{\hat{B}}(l)+\eta\right) .
$$

The identification of $\mathcal{F}(Y, \mathcal{O})$ with $D^{b}(A \times B)$ is given by the restriction to $A \times\{0\} \times$ $B \times\{0\} \subset X_{A} \times X_{B}$ (see Example 2.3.2). Since $p_{A B}: L \rightarrow A \times B$ is surjective, the map $q$ is also surjective. Therefore, we can use the base change formula to get the required expression for $S_{L, \alpha}$ as an object of $D^{b}(A \times B)$.

(ii) This follows from Proposition 2.4 .12 by a similar argument. Note that we can still use the base change formula since the image of $q$ is transversal to the subvariety $A \times\{0\} \times$ $B \times\{0\} \subset X_{A} \times X_{B}$. 
Examples 3.2.6. 1. According to [18] any equivalence between $D^{b}(A)$ and $D^{b}(B)$ appears as an LI-functor associated with the graph $L(f)$ of a symplectic isomorphism $f: X_{A} \simeq X_{B}$ (see Example 3.1.2). More precisely, to construct such an equivalence one uses the natural equivalence of $D^{b}(A)$ with the representation $\mathcal{F}(Y, \alpha)$ of the Heisenberg groupoid $\mathbf{H}\left(X_{B}\right)$ of $X_{B}$ associated with the Lagrangian pair $(Y, \alpha)$ that corresponds to $\{0\} \times \hat{A}$ under the isomorphism $f$. Now Theorem [20, Thm. 4.3] gives an equivalence of $\mathcal{F}(Y, \alpha)$ with $D^{b}(B)$. Note that the above construction depends on a choice of an extension of $f$ to an equivalence of Heisenberg groupoids $\mathbf{H}\left(X_{A}\right) \simeq \mathbf{H}\left(X_{B}\right)$, and the obtained equivalence $D^{b}(A) \simeq D^{b}(B)$ is compatible with the action of these groupoids. This implies that the corresponding kernel $K$ on $A \times B$ belongs to $D^{b}(A \times B)^{(L(f), \beta)}$, where $(L(f)$, $\beta)$ is some Lagrangian correspondence extending the graph $L(f)$. Furthermore, by [18, Prop. $3.2], K$ is cohomologically pure. Hence, by Theorem $2.4 .5, K$ is a direct sum of several copies of $S_{L(f), \beta}$, which implies that $K \simeq S_{L(f), \beta}$ (since the corresponding functor is an equivalence). For example, the Fourier-Mukai transform $\mathcal{S}: D^{b}(A) \rightarrow D^{b}(\hat{A})$ is associated with the Lagrangian $\Gamma_{\mathcal{S}} \subset X_{A} \times X_{\hat{A}}=A \times \hat{A} \times \hat{A} \times A$ consisting of $(x, \xi, \xi,-x)$, where $x \in A, \xi \in \hat{A}$. Note that in the case $A=B$ autoequivalences corresponding to symplectic automorphisms of $X_{A}$ were also considered in [19] and [15].

2. Let $f: A \rightarrow B$ be a homomorphism. Then with $f$ we can associate a Lagrangian correspondence from $X_{A}$ to $X_{B}$ by setting $L=A \times \hat{B}$, where the map $L \rightarrow B$ is induced by $f$ and the map $L \rightarrow \hat{A}$ is induced by $\hat{f}$. In this case we can take $\alpha$ to be trivial. Then $L$-invariants in $D^{b}(A \times B)$ are generated by the structure sheaf of the graph of $f$. The corresponding LI-functor $D^{b}(A) \rightarrow D^{b}(B)$ is the derived push-forward $R f_{*}$. The functor corresponding to the opposite Lagrangian is the pull-back $L f^{*}: D^{b}(B) \rightarrow D^{b}(A)$.

The following proposition shows that the adjoint functors to gLI-functors are also gLIfunctors.

Proposition 3.2.7. Let $(L, \alpha)$ be a g-Lag-correspondence from $X_{A}$ to $X_{B}$, and consider the permutation maps

$$
\sigma: A \times B \rightarrow B \times A \text { and } \sigma_{X}: X_{A} \times X_{B} \rightarrow X_{B} \times X_{A}
$$

Then the objects $S_{L, \alpha}$ and $\sigma^{*} S_{\sigma_{X}(L), \alpha^{-1}}$ in $D^{b}(A \times B)$ are dual up to a shift. Hence, the functors $\Phi_{L, \alpha}: D^{b}(A) \rightarrow D^{b}(B)$ and $\Phi_{\sigma L, \alpha^{-1}}: D^{b}(B) \rightarrow D^{b}(A)$ are adjoint up to a shift.

Proof. Consider the duality functor

$$
D: D^{b}(A \times B) \rightarrow D^{b}(A \times B): K \mapsto R \underline{\operatorname{Hom}}(K, \mathcal{O}) .
$$

We have

$$
T_{(a, \xi, b, \eta)}(D(K)) \simeq D\left(T_{(a,-\xi, b,-\eta)}(K)\right)
$$

for $(a, \xi, b, \eta) \in X_{A} \times X_{B}$. On the other hand, for $K^{\prime} \in D^{b}(B \times A)$ we have

$$
T_{(a,-\xi, b,-\eta)}\left(\sigma^{*} K^{\prime}\right) \simeq \sigma^{*}\left(T_{(b, \eta, a, \xi)} K^{\prime}\right) .
$$

Combining these isomorphisms we obtain

$$
T_{u}\left(D\left(\sigma^{*} K^{\prime}\right)\right) \simeq D\left(\sigma^{*}\left(T_{\sigma_{X}(u)} K^{\prime}\right)\right),
$$

where $u \in X_{A} \times X_{B}$. This shows that $D\left(\sigma^{*} S_{\sigma_{X}(L), \alpha^{-1}}\right)$ has an $(L, \alpha)$-invariant structure. 
Our main goal is to calculate convolutions of gLI-kernels (and hence compositions of gLI-functors). We will need the following technical result for this.

Lemma 3.2.8. Let $X$ be a commutative group scheme equipped with homomorphisms $f: X \rightarrow A, g: X \rightarrow \hat{A}$, where $A$ is an abelian variety. Assume that both $f$ and $g$ are surjective with finite kernel. Assume also that $\mathcal{L}$ is a line bundle on $X$ such that

$$
\Lambda(\mathcal{L}) \simeq(f \times g)^{*} \mathcal{P}_{A} .
$$

Then the restriction of $\mathcal{L}$ to every connected component of $X$ is nondegenerate of the same index $i_{\mathcal{L}}$, so $H^{i}(X, \mathcal{L})=0$ for $i \neq i_{\mathcal{L}}$. Furthermore,

$$
\begin{aligned}
& \left(\operatorname{dim} H^{i_{\mathcal{L}}}(X, \mathcal{L})\right)^{2}=|\operatorname{ker}(f)| \cdot|\operatorname{ker}(g)| \text { and } \\
& i_{\mathcal{L}}=i\left(\hat{f}_{0} \circ g_{0}\right), \text { where } f_{0}=\left.f\right|_{X_{0}}, g_{0}=\left.g\right|_{X_{0}}
\end{aligned}
$$

(here we use the notation of Sec. 1.3).

Proof. Set $G=\operatorname{ker}(g), H=\operatorname{ker}(f)$, and let $G_{0}=G \cap X_{0}, H_{0}=H \cap X_{0}$. Then we have exact sequences

$$
\begin{aligned}
& 0 \rightarrow G_{0} \rightarrow X_{0} \stackrel{g_{0}}{\longrightarrow} \hat{A} \rightarrow 0 \\
& 0 \rightarrow H_{0} \rightarrow X_{0} \stackrel{f_{0}}{\longrightarrow} A \rightarrow 0 .
\end{aligned}
$$

Also, we have $\pi_{0}(X) \simeq G / G_{0} \simeq H / H_{0}$. The isomorphism (3.2.2) implies that for every point $x \in H$ one has $t_{x}^{*} \mathcal{L} \simeq \mathcal{L}$. Since $H$ surjects onto $\pi_{0}(X)$, this implies that the restrictions of $\mathcal{L}$ to all connected components of $X$ are obtained from $\left.\mathcal{L}\right|_{X_{0}}$ by translations. Hence,

$$
H^{*}(X, \mathcal{L}) \simeq H^{*}\left(X_{0},\left.\mathcal{L}\right|_{X_{0}}\right)^{\oplus\left|\pi_{0}(X)\right|} .
$$

On the other hand, the isomorphism (3.2.2) induces an isomorphism

$$
\Lambda\left(\left.\mathcal{L}\right|_{X_{0}}\right) \simeq\left(f_{0} \times g_{0}\right)^{*} \mathcal{P}_{A}
$$

Therefore, the homomorphism $\phi: X_{0} \rightarrow \widehat{X_{0}}$ associated with $\left.\mathcal{L}\right|_{X_{0}}$ is equal to the composition

$$
X_{0} \stackrel{f_{0}}{\longrightarrow} A \stackrel{\widehat{g_{0}}}{\longrightarrow} \widehat{X_{0}} .
$$

Therefore, the group $K:=K\left(\left.\mathcal{L}\right|_{X_{0}}\right)=\operatorname{ker}(\phi)$ fits into an exact sequence

$$
0 \rightarrow H_{0} \rightarrow K \rightarrow G_{0} \rightarrow 0 .
$$

Hence, $K$ is finite, i.e., the line bundle $\left.\mathcal{L}\right|_{X_{0}}$ is nondegenerate of some index $i=i_{\mathcal{L}}$. It remains to observe that

$$
|K|=\left|H_{0}\right| \cdot\left|G_{0}\right|=\frac{|H| \cdot|G|}{\left|\pi_{0}(X)\right|^{2}}
$$

and so from (3.2.3) we obtain

$$
\operatorname{dim} H^{i}(X, \mathcal{L})=\left|\pi_{0}(X)\right| \cdot|K|^{1 / 2}=|H|^{1 / 2} \cdot|G|^{1 / 2} .
$$

The formula for the index $i_{\mathcal{L}}$ follows from the fact that $\phi_{\mathcal{L}}=\hat{f}_{0} \circ g_{0}$.

In the next proposition we calculate convolutions of gLI-kernels under some technical nondegeneracy assumptions. 
Proposition 3.2.9. (i) Let $(L, \alpha)$ (resp., $(M, \beta)$ ) be a g-Lag-correspondence from $X_{A}$ to $X_{B}$ (resp., from $X_{B}$ to $X_{C}$ ). Assume that the natural homomorphism $L \times M \rightarrow X_{B}$ (restricting to the given ones on $L$ and $M)$ is surjective. Then for an $(L, \alpha)$-invariant object $F \in D^{b}(A \times B)$ and an $(M, \beta)$-invariant object $G \in D^{b}(B \times C)$ the convolution $F \circ_{B} G$ (see Sec. 1.5) can be equipped with a $(M \circ L, \beta \circ \alpha)$-invariance structure.

(ii) Assume in addition that the $g$-Lag-correspondences $L, M$ and $M \circ L$ are nondegenerate. Then one has an isomorphism in $D^{b}(A \times C)$

$$
S_{L, \alpha} \circ_{B} S_{M, \beta} \simeq S_{M \circ L, \beta \circ \alpha}[\lambda(L, M)],
$$

and hence an isomorphism of functors

$$
\Phi_{M, \beta} \circ \Phi_{L, \alpha} \simeq \Phi_{M \circ L, \beta \circ \alpha}[\lambda(L, M)] .
$$

Here the integer $\lambda(L, M) \leq 0$ is defined as follows. Let us set

$$
F=\operatorname{ker}\left(L \times_{B} M \rightarrow A \times C:(l, m) \mapsto\left(p_{A}(l), p_{C}(m)\right)\right) .
$$

Consider the maps

$$
f: F \rightarrow B \text { and } g: F \rightarrow \hat{B},
$$

where $f$ is the restriction by the projection to $B$ and $g$ is the restriction of the map

$$
L \times_{B} M \rightarrow \hat{B}:(l, m) \mapsto p_{\hat{B}}(l)-p_{\hat{B}}(m) .
$$

Set $f_{0}=\left.f\right|_{F_{0}}$ and $g_{0}=\left.g\right|_{F_{0}}$. Then both $f_{0}$ and $g_{0}$ are isogenies, $\hat{f}_{0} \circ g_{0}$ is symmetric and we set

$$
\lambda(L, M)=-i\left(\hat{f}_{0} \circ g_{0}\right) .
$$

Proof. (i) For $\mathcal{F} \in D^{b}(A \times B), \mathcal{G} \in D^{b}(B \times C)$ let us set

$$
\mathcal{F} \diamond \mathcal{G}=p_{12}^{*} \mathcal{F} \otimes^{\mathbb{L}} p_{23}^{*} \mathcal{G} \in F^{b}(A \times B \times C) .
$$

We have

$$
\begin{gathered}
\left(t_{(a, b)}^{*} \mathcal{F}\right) \diamond\left(t_{(b, c)}^{*} \mathcal{G}\right) \simeq t_{(a, b, c)}^{*}(\mathcal{F} \diamond \mathcal{G}) \text { and } \\
\left(\mathcal{F} \otimes\left(\mathcal{P}_{-\xi} \otimes \mathcal{P}_{\eta}\right)\right) \diamond\left(\mathcal{G} \otimes\left(\mathcal{P}_{-\eta} \otimes \mathcal{P}_{\rho}\right)\right)=(\mathcal{F} \diamond \mathcal{G}) \otimes\left(\mathcal{P}_{-\xi} \otimes \mathcal{O}_{B} \otimes \mathcal{P}_{\rho}\right),
\end{gathered}
$$

where $(a, b, c) \in A \times B \times C$ and $(\xi, \eta, \rho) \in \hat{A} \times \hat{B} \times \hat{C}$. Hence, for $(l, m) \in L \times_{X_{B}} M$, $\mathcal{F} \in D^{b}(A \times B)^{(L, \alpha)}$ and $\mathcal{G} \in D^{b}(B \times C)^{(M, \beta)}$ we obtain an isomorphism

$$
\mathcal{F} \diamond \mathcal{G} \simeq T_{l}(\mathcal{F}) \diamond T_{m}(\mathcal{G}) \simeq\left(\mathbb{T}_{p_{A \hat{A}}(l)}^{A}\right)^{-1} \circ_{A} t_{b(l, m)}^{*}(\mathcal{F} \diamond \mathcal{G}) \circ_{C} \mathbb{T}_{p_{C \hat{C}}(m)}^{C},
$$

where $b(l, m)=p_{B}(l)=p_{B}(m)$. Consider the extension of the canonical map $L \times_{X_{B}} M \rightarrow$ $X_{A} \times X_{C}$ to a map $i: L \times_{X_{B}} M \rightarrow X_{A} \times B \times X_{C} \subset X_{A} \times X_{B} \times X_{C}$ with the $B$-component given by $b(l, m)$. We can view in this way $(M \circ L, \beta \circ \alpha)$ as a generalized isotropic pair for $X_{A} \times X_{B} \times X_{C}$ equipped with the (degenerate) biextension $\mathcal{B}_{A}^{-1} \otimes \mathcal{O}_{X_{B}} \otimes \mathcal{B}_{C}$. The above calculation shows that $\mathcal{F} \diamond \mathcal{G}$ has an $(M \circ L, \beta \circ \alpha)$-invariance structure. This immediately leads to the required invariance structure on the push-forward of $\mathcal{F} \diamond \mathcal{G}$ to $D(A \times C)$, i.e., on $\mathcal{F} \circ_{B} \mathcal{G}$.

(ii) Let $p_{A B}: L \rightarrow A \times B, p_{B C}: M \rightarrow B \times C$ and $p_{A C}: L \circ M \rightarrow A \times C$ denote the natural projections. Recall that

$$
\widetilde{S}_{L, \alpha}:=p_{A B *}\left(\alpha^{-1} \otimes p_{A \hat{A}}^{*} \mathcal{P}_{37}^{-1} \otimes p_{B \hat{B}}^{*} \mathcal{P}\right) \simeq V_{L} \otimes S_{L, \alpha},
$$




$$
\widetilde{S}_{M, \beta}:=p_{B C *}\left(\beta^{-1} \otimes p_{B \hat{B}}^{*} \mathcal{P}^{-1} \otimes p_{C \hat{C}}^{*} \mathcal{P}\right) \simeq V_{M} \otimes S_{M, \beta},
$$

where $V_{L}$ and $V_{M}$ are vector spaces of ranks $\left|\operatorname{ker}\left(p_{A B}\right)\right|^{1 / 2}$ and $\left|\operatorname{ker}\left(p_{B C}\right)\right|^{1 / 2}$, respectively. Also, $S_{M \circ L, \beta \circ \alpha}$ is a vector bundle of rank $\left|\operatorname{ker}\left(p_{A C}\right)\right|^{1 / 2}$. Thus, by part (i) and Theorem 2.4.5, it suffices to check that

$$
\widetilde{S}_{L, \alpha} \circ_{B} \widetilde{S}_{M, \beta} \simeq \mathcal{V}\left[-i\left(\hat{f}_{0} \circ g_{0}\right]\right.
$$

where $\mathcal{V}$ is a vector bundle on $A \times C$ of rank

$$
\left|\operatorname{ker}\left(p_{A C}\right)\right|^{1 / 2} \cdot\left|\operatorname{ker}\left(p_{A B}\right)\right|^{1 / 2} \cdot\left|\operatorname{ker}\left(p_{B C}\right)\right|^{1 / 2} .
$$

Using the commutative diagram with cartesian squares

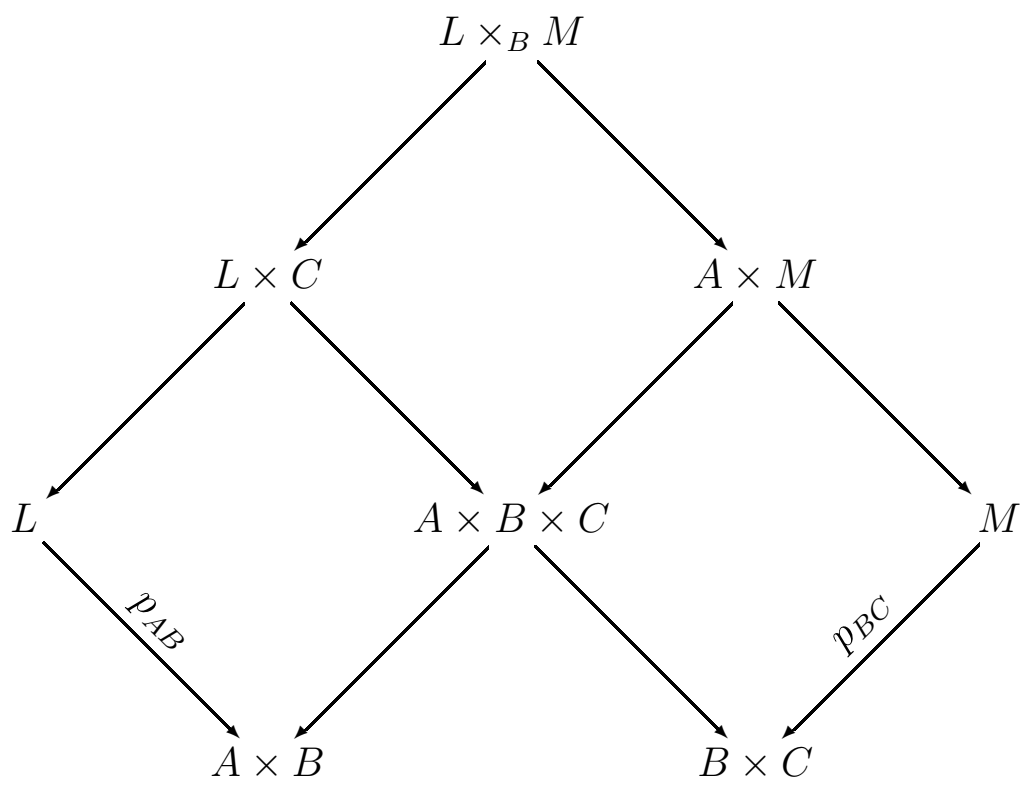

and applying base change and projection formulas we find an isomorphism

$$
\begin{aligned}
& \widetilde{S}_{L, \alpha} \circ_{B} \widetilde{S}_{M, \beta} \simeq \\
& p_{A B *}\left(\alpha^{-1} \otimes p_{A \hat{A}}^{*} \mathcal{P}^{-1} \otimes p_{B \hat{B}}^{*} \mathcal{P}\right) \circ_{B} p_{B C *}\left(\beta^{-1} \otimes p_{B \hat{B}}^{*} \mathcal{P}^{-1} \otimes p_{C \hat{C}}^{*} \mathcal{P}\right) \simeq p_{A C *}^{\prime}(\mathcal{L}),
\end{aligned}
$$

where $p_{A C}^{\prime}: L \times_{B} M \rightarrow A \times C$ is the projection and

$$
\mathcal{L}=\alpha^{-1} \otimes \beta^{-1} \otimes p_{A \hat{A}}^{*} \mathcal{P}^{-1} \otimes p_{C \hat{C}}^{*} \mathcal{P}^{-1} \otimes p_{B \hat{B} \hat{B}}^{*}\left(\operatorname{id}_{B} \times \delta\right)^{*} \mathcal{P}
$$

with $\delta: \hat{B} \times \hat{B} \rightarrow \hat{B}$ given by $\delta\left(\xi_{1}, \xi_{2}\right)=\xi_{1}-\xi_{2}$. It is easy to see that

$$
\Lambda(\mathcal{L})_{\left(l_{1}, m_{1}\right),\left(l_{2}, m_{2}\right)} \simeq \mathcal{P}_{p_{A}\left(l_{2}\right), p_{\hat{A}}\left(l_{1}\right)}^{-1} \otimes \mathcal{P}_{p_{C}\left(m_{2}\right), p_{\hat{C}}\left(m_{1}\right)} \otimes \mathcal{P}_{p_{B}\left(l_{2}\right), p_{\hat{B}}\left(l_{1}\right)-p_{\hat{B}}\left(m_{1}\right)} .
$$

Let us consider the fiber $F=\operatorname{ker}\left(p_{A C}^{\prime}: L \times_{B} M \rightarrow A \times C\right)$. Then the above formula specializes to

$$
\left.\Lambda(\mathcal{L})\right|_{F \times F} \simeq(f \times g)^{*} \mathcal{P} .
$$

The diagram (3.2.5) implies that the projection $L \times_{B} M \rightarrow A \times B \times C$ is surjective with a finite kernel $G$, where

$$
|G|=\operatorname{deg}\left(p_{A B}\right) \cdot \operatorname{deg}\left(p_{B C}\right)=\left|\operatorname{ker}\left(p_{A B}\right)\right| \cdot\left|\operatorname{ker}\left(p_{B C}\right)\right| .
$$


Hence, the projection $f: F \rightarrow B$ is also surjective with the kernel isomorphic to $G$. On the other hand, the subgroup $H=\operatorname{ker}(g) \subset F$ can be identified with

$$
\left(L \times_{X_{B}} M\right) \cap F=\operatorname{ker}\left(p_{A C}: M \circ L \rightarrow A \times C\right),
$$

where we view $M \circ L=L \times_{X_{B}} M$ as a subgroup in $L \times_{B} M$. Recall that by assumption, $p_{A C}$ is surjective with finite kernel, so $g: F \rightarrow \hat{B}$ is surjective with finite kernel. Now Lemma 3.2.8 implies that $p_{A C *}^{\prime}(\mathcal{L})$ is of the form $\mathcal{V}\left[-i\left(\hat{f}_{0} \circ g_{0}\right]\right.$ for some vector bundle $\mathcal{V}$ of rank

$$
|G|^{1 / 2} \cdot|H|^{1 / 2}=\left|\operatorname{ker}\left(p_{A C}\right)\right|^{1 / 2} \cdot\left|\operatorname{ker}\left(p_{A B}\right)\right|^{1 / 2} \cdot\left|\operatorname{ker}\left(p_{B C}\right)\right|^{1 / 2},
$$

as required.

To get rid of the nondegeneracy assumption in Proposition 3.2.9 we will use twisting by autoequivalences. Namely, we will use the fact that in the case when $L=L(f) \subset X_{A} \times X_{A}$ is a graph of a symplectic automorphism $f: X_{A} \rightarrow X_{A}$ the corresponding functor $\Phi_{L(f), \alpha}$ is an equivalence (see Example 3.2.6.1).

Lemma 3.2.10. Let $(L, \alpha)$ be a g-Lag-correspondence from $X_{A}$ to $X_{B}$, and let $f: X_{A} \rightarrow$ $X_{A}$ (resp., $g: X_{B} \rightarrow X_{B}$ ) be a symplectic automorphism. Let also $\left(L(f), \alpha_{f}\right)$ (resp., $\left(L(g), \alpha_{g}\right)$ ) be a Lagrangian correspondence from $X_{A}$ to $X_{A}$ (resp. from $X_{B}$ to $X_{B}$ ) extending the graph of $f$ (resp., g). Then

$$
S_{L(f), \alpha_{f}} \circ_{A} S_{L, \alpha} \circ_{B} S_{L(g), \alpha_{g}} \simeq S_{L(g) \circ L \circ L(f), \alpha_{g} \circ \alpha \circ \alpha_{f}}[i]
$$

for some $i \in \mathbb{Z}$, and

$$
L(g) \circ L \circ L(f)=\left(f^{-1} \times g\right)(L) .
$$

Proof. Note that (3.2.7) follows immediately from the definition of the composition of correspondences. Let us set for brevity $S_{f}=S_{L(f), \alpha_{f}}, S_{g}=S_{L(g), \alpha_{g}}, S=S_{L, \alpha}$ and $S^{\prime}=S_{L(g) \circ L \circ L(f), \alpha_{g} \circ \alpha \circ \alpha_{f}}$. Let $S_{f^{-1}}$ denote the kernel of the inverse autoequivalence to the one defined by $S_{f}$, so that

$$
S_{f^{-1}} \circ_{A} S_{f} \simeq \Delta_{*} \mathcal{O}_{A}
$$

Then $S_{f^{-1}}$ has a $\left(L\left(f^{-1}\right), \sigma^{*} \alpha_{f}^{-1}\right)$-invariance structure, where $\sigma: L\left(f^{-1}\right) \rightarrow L(f)$ is the isomorphism induced by the permutation of factors in $X_{A} \times X_{A}$, so $S_{f^{-1}}$ is isomorphic to $S_{L\left(f^{-1}\right), \sigma^{*} \alpha_{f}^{-1}}[m]$ for some $m \in \mathbb{Z}$. Similarly, for $S_{g^{-1}}=S_{L\left(g^{-1}\right), \sigma^{*} \alpha_{g}^{-1}}[n]$, where $n \in \mathbb{Z}$, we have

$$
S_{g} \circ_{B} S_{g^{-1}} \simeq \Delta_{*} \mathcal{O}_{B}
$$

Now by Proposition 3.2.9(i), the object

$$
P:=S_{f} \circ_{A} S \circ_{B} S_{g} \in D^{b}(A \times B)
$$

has a structure of an object of $D^{b}(A \times A)^{\left(L(g) \circ L \circ L(f), \alpha_{g} \circ \alpha \circ \alpha_{f}\right)}$. Similarly the object

$$
Q:=S_{f^{-1}} \circ_{A} S^{\prime} \circ_{B} S_{g^{-1}}
$$

has a structure of an object of $D^{b}(A \times B)^{(L, \alpha)}$. Let $[a, b]$ (resp., $\left.[s, t]\right)$ be the cohomological amplitude of $P$ (resp., $Q$ ). Then by Theorem 2.4.5, we have an exact triangle

$$
\tau_{<b} P \rightarrow P \rightarrow \underset{39}{V \otimes S^{\prime}[-b] \rightarrow \ldots,}
$$


where $V$ is a vector space and $\tau_{<b} P$ is a successive extension of $S^{\prime}[-i]$ with $i<b$. Applying the convolution with $S_{f^{-1}}$ on the left and with $S_{g^{-1}}$ on the right we obtain an exact triangle

$$
S_{f^{-1}} \circ_{A} \tau_{<b} P \circ_{B} S_{g^{-1}} \rightarrow S \rightarrow V \otimes Q[-b] \rightarrow \ldots
$$

where $S_{f^{-1}} \circ_{A} \tau_{<b} P \circ_{B} S_{g^{-1}}$ is a successive extension of $Q[-i]$ with $i<b$. It follows that

$$
\underline{H}^{b+t}(S) \simeq V \otimes \underline{H}^{t}(Q) \neq 0 \text {. }
$$

A similar argument shows that $\underline{H}^{a+s}\left(S^{\prime}\right) \neq 0$. Hence, $b+t=a+s=0$ which implies that $b=a$ and $s=t=-a$. Thus, we have

$$
P \simeq V \otimes S^{\prime}[-a] \text { and } Q \simeq W \otimes S[a]
$$

for some vector spaces $V$ and $W$. Therefore, we obtain

$$
S \simeq S_{f^{-1}} \circ_{A} P \circ_{B} S_{g^{-1}} \simeq V \otimes Q[-a] \simeq V \otimes W \otimes S,
$$

which implies that $V$ and $W$ are one-dimensional.

Now we are ready to prove our main result about the convolution of gLI-kernels.

Theorem 3.2.11. Let $(L, \alpha)$ (resp., $(M, \beta)$ ) be a g-Lag-correspondence from $X_{A}$ to $X_{B}$ (resp., from $X_{B}$ to $X_{C}$ ). Assume that the natural homomorphism $L \times M \rightarrow X_{B}$ is surjective. Then one has an isomorphism in $D^{b}(A \times C)$

$$
S_{L, \alpha} \circ_{B} S_{M, \beta} \simeq S_{M \circ L, \beta \circ \alpha}[i] \text { for some } i \in \mathbb{Z},
$$

and hence an isomorphism of functors

$$
\Phi_{M, \beta} \circ \Phi_{L, \alpha} \simeq \Phi_{M \circ L, \beta \circ \alpha}[i] .
$$

Proof. The idea is to reduce to the case when the correspondences $L, M$ and $M \circ L$ are nondegenerate, considered in Proposition 3.2.9(ii). Let $\phi_{A}: \hat{A} \rightarrow A, \phi_{B}: \hat{B} \rightarrow B$ and $\phi_{C}: \hat{C} \rightarrow C$ be symmetric isogenies associated with some ample line bundles on $\hat{A}, \hat{B}$ and $\hat{C}$. For any $n \in \mathbb{Z}$ let us define the symplectic automorphism $f_{A}(n)$ of $X_{A}=A \times \hat{A}$ by

$$
f_{A}(n)=\left(\begin{array}{cc}
\operatorname{id}_{A} & n \phi_{A} \\
0 & \operatorname{id}_{\hat{A}}
\end{array}\right)
$$

Similarly, we define symplectic automorphisms $f_{B}(n)$ of $X_{B}$ and $f_{C}(n)$ of $X_{C}$. We claim that for all $n$ except for a finite number of values the correspondence $\left(f_{A}(n) \times f_{B}(n)\right)(L)$ is nondegenerate. Indeed, by definition, nondegeneracy means transversality to the Lagrangian subvariety $\{0\} \times \hat{A} \times\{0\} \times \hat{B} \subset X_{A} \times X_{B}$. Thus, we need $n$ such that $L$ is transversal to

$$
\left(f_{A}(-n) \times f_{B}(-n)\right)(\{0\} \times \hat{A} \times\{0\} \times \hat{B})=\Gamma\left(-n\left(\phi_{A} \times \phi_{B}\right)\right),
$$

and our claim follows from Lemma 2.2.7(ii). Similarly, for generic $n$ the correspondences $\left(f_{B}(n) \times f_{C}(n)\right)(M)$ and $\left(f_{A}(n) \times f_{C}(n)\right)(M \circ L)$ are nondegenerate. Let us choose such $n$ and extend $f_{A}=f_{A}(n), f_{B}=f_{B}(n)$ and $f_{C}=f_{C}(n)$ to some Lagrangian correspondences $\left(L\left(f_{A}\right), \alpha_{1}\right),\left(L\left(f_{B}\right), \alpha_{2}\right)$ and $\left(L\left(f_{C}\right), \alpha_{3}\right)$. Let also $S_{f_{A}}, S_{f_{A}^{-1}}$, etc., be the kernels giving the corresponding autoequivalences and their inverses. By Lemma 3.2.10, we have

$$
S_{L} \simeq S_{f_{A}} \circ_{A} S_{\left(f_{A} \times f_{B}\right)(L)} \circ_{B} S_{f_{B}^{-1}}[a] \text { and }
$$




$$
S_{M} \simeq S_{f_{B}} \circ_{B} S_{\left(f_{B} \times f_{C}\right)(M)} \circ_{C} S_{f_{C}^{-1}}[b]
$$

for some $a, b \in \mathbb{Z}$, where we abbreviate $S_{L, \alpha}$ to $S_{L}$, etc.. Therefore,

$$
S_{L} \circ_{B} S_{M} \simeq S_{f_{A}} \circ_{A} S_{\left(f_{A} \times f_{B}\right)(L)} \circ_{B} S_{\left(f_{B} \times f_{C}\right)(M)} \circ_{C} S_{f_{C}^{-1}}[a+b] .
$$

Now Proposition 3.2.9(ii) allows to compute the convolution of kernels in the middle, so we obtain

$$
S_{L} \circ_{B} S_{M} \simeq S_{f_{A}} \circ_{A} S_{\left(f_{A} \times f_{C}\right)(M \circ L)} \circ_{C} S_{f_{C}^{-1}}[c]
$$

for some $c \in \mathbb{Z}$. By Lemma 3.2.10, the right-hand side is isomorphic to $S_{M \circ L}[i]$ for some $i \in \mathbb{Z}$.

Corollary 3.2.12. Let $(L, \alpha)$ and $(M, \beta)$ be generalized Lagrangian pairs for the standard ess-abelian variety $X_{A}$. Assume that $L$ and $M$ are transversal, and let $G$ be the corresponding Heisenberg extension of $L \times_{X_{A}} M$ (see Proposition 2.2.8), with the underlying $\mathbb{G}_{m}$-torsor given by the tensor product of the pull-backs of $\alpha^{-1}$ and $\beta$. Then $\operatorname{Hom}_{D^{b}(A)}^{*}\left(S_{L, \alpha}, S_{M, \beta}\right)$ is concentrated in one degree and is an irreducible representation of $G$ of weight one.

Proof. We have

$$
\operatorname{Hom}^{*}\left(S_{L, \alpha}, S_{M, \beta}\right) \simeq H^{*}\left(A, S_{L, \alpha}^{\vee} \otimes^{\mathbb{L}} S_{M, \beta}\right)
$$

Now we interpret $(L, \alpha)$ and $(M, \beta)$ as correspondences from 0 to $X_{A}$ and use Proposition 3.2.7 that says that $S_{L, \alpha}^{\vee}$ is isomorphic to a shift of the LI-kernel corresponding to $\left(L, \alpha^{-1}\right)$, viewed as a correspondence from $X_{A}$ to 0 . Now we can compute $H^{*}\left(A, S_{L, \alpha}^{\vee} \otimes S_{M, \beta}\right)$ by applying Theorem 3.2.11. Note that the composition of correspondences in our case is $L \times_{X_{A}} M$, and $\beta \circ \alpha^{-1}$ is exactly the underlying $\mathbb{G}_{m}$-torsor of $G$. Thus, $\left(M \circ \bar{L}, \beta \circ \alpha^{-1}\right)$ invariance structure on a vector space can be viewed as a weight-1 representation of the Heisenberg extension $G$ (see Example 2.2.4.3 and Sec. 1.1).

Remark 3.2.13. Corollary 3.2.12 generalizes the well known result that for a nondegenerate line bundle $L$ on $A$ the cohomology $H^{*}(A, L)$ is concentrated in one degree and is an irreducible weight-1 representation of the Mumford's theta group attached to $L$ (see [16, Sec. 16 and 23]). Note that actions of some natural groups on the cohomology of vector bundles on $A$ were also considered by Umemura [26].

For $g=\left(\begin{array}{ll}a & b \\ c & d\end{array}\right) \in U\left(X_{A}, \mathbb{Q}\right)$ let us denote $b=b(g) \in \operatorname{Hom}(\hat{A}, A)_{\mathbb{Q}}$. Let us consider the subset $U^{0} \subset U\left(X_{A}, \mathbb{Q}\right)$ consisting of $g$ such that $b(g)$ is invertible. Note that the group $U\left(X_{A}, \mathbb{Q}\right)$ is completely determined by the algebra $R=\operatorname{End}(A) \otimes \mathbb{Q}$ and the Rosati involution $\iota$ on it (with respect to some polarization). Namely, it consists of automorphisms of the free rank-2 module over $R$ preserving the standard skew-Hermitian form $\left(\left(x_{1}, y_{1}\right),\left(x_{2}, y_{2}\right)\right)=\iota\left(x_{1}\right) y_{2}-\iota\left(x_{2}\right) y_{1}$. It follows that $U\left(X_{A}, \mathbb{Q}\right)$ can be identified with the group of $\mathbb{Q}$-points in a connected algebraic group $U_{X_{A}}$ (see [19, Sec. 9] and [21, Sec. 4] for a more detailed study of the group $U_{X_{A}}$, which is denoted there by $\mathrm{SL}_{2, A, \mathbb{Q}}$ ). Since $U\left(X_{A}, \mathbb{Q}\right)$ is Zariski dense in $U_{X_{A}}$ (see $[1,18.3]$ ) and since the invertibility of $b(g)$ is a Zariski open condition, we deduce that the subset $U^{0} \subset U\left(X_{A}, \mathbb{Q}\right)$ is big in the following sense: for any triple of elements $g_{1}, g_{2}, g_{3} \in U\left(X_{A}, \mathbb{Q}\right)$ the intersection $\left(U^{0}\right)^{-1} \cap U^{0} g_{1} \cap$ 
$U^{0} g_{2} \cap U^{0} g_{3}$ is non-empty (this notion goes back to [27, IV. 42] while the term is due to D. Kazhdan). The importance of this condition is due to the fact that a 2-cocycle of $U\left(X_{A}, \mathbb{Q}\right)$ is uniquely determined by its restriction to $U^{0} \times U^{0}$ (see [21, Lem. 4.2]).

Recall that we denote by $\operatorname{Lag}\left(X_{A}\right)$ the monoid of g-Lag-correspondences $(L, \alpha)$ from $X_{A}$ to $X_{A}$ such that both projections $L \rightarrow X_{A}$ are surjective. We have a surjective homomorphism $\pi: \operatorname{Lag}\left(X_{A}\right) \rightarrow U\left(X_{A}, \mathbb{Q}\right)$ (see Lemma 3.1.6). Note that a g-Lag-correspondence $L$ is nondegenerate if and only if $\pi(L) \in U^{0}$ (see Example 3.2.2). Proposition 3.2.9 and Theorem 3.2.11 lead to the following computation of the convolution of gLI-kernels (and hence the composition of gLI-functors) associated with g-Lag-correspondences from $\operatorname{Lag}\left(X_{A}\right)$.

Theorem 3.2.14. (i) For any pair of g-Lag-correspondences $(L, \alpha),(M, \beta) \in \operatorname{Lag}\left(X_{A}\right)$ we have

$$
S_{L, \alpha} \circ_{A} S_{M, \beta} \simeq S_{M \circ L, \beta \circ \alpha}[\lambda(\pi(M), \pi(L))]
$$

for some integer $\lambda(\pi(L), \pi(M))$. Hence, we also have an isomorphism of functors

$$
\Phi_{M, \beta} \circ \Phi_{L, \alpha} \simeq \Phi_{M \circ L, \beta \circ \alpha}[\lambda(L, M)] .
$$

(ii) The map $\lambda\left(g_{1}, g_{2}\right)$ is a 2-cocycle of $U\left(X_{A}, \mathbb{Q}\right)$ with values in $\mathbb{Z}$. We have

$$
\lambda\left(g_{1}, g_{2}\right)=-i\left(b\left(g_{1}\right)^{-1} b\left(g_{1} g_{2}\right) b\left(g_{2}\right)^{-1}\right)
$$

whenever $b\left(g_{1}\right), b\left(g_{2}\right)$ and $b\left(g_{1} g_{2}\right)$ are invertible. Here $i(\cdot)$ denotes the index of a symmetric isogeny (see Section 1.3).

Proof. (i) Theorem 3.2.11 implies that

$$
S_{L, \alpha} \circ_{A} S_{M, \beta} \simeq S_{M \circ L, \beta \circ \alpha}[\lambda(L, M)]
$$

for some integer $\lambda(L, M)$. We have to prove that $\lambda(L, M)$ depends only on $\pi(L)$ and $\pi(M)$. Let $g=\pi(L)$ and let us equip $L(g) \subset X_{A} \times X_{A}$ with a line bundle $\alpha_{g}$ so that $\left(L(g), \alpha_{g}\right)$ is a Lagrangian correspondence. By Proposition 2.4.7(ii), $S_{L, \alpha}$ is obtained by successive extensions from objects of the form $T_{x}\left(S_{L(g), \alpha_{g}}\right)$ where $x \in X_{A \times A}$. Using Lemmas 3.2.3 and 3.2.4, we can rewrite such objects as $\mathbb{T}_{x^{\prime} \circ_{A}} S_{L(g), \alpha_{g}}$ with $x^{\prime} \in X_{A}$. This immediately implies that $S_{L, \alpha} \circ_{A} S_{M, \beta}$ is obtained by successive extensions from objects of the form $\mathbb{T}_{x^{\prime} \circ_{A}} S_{L(g), \alpha_{g}} \circ_{A} S_{M, \beta}$, hence $\lambda(L, M)=\lambda(L(g), M)$. A similar argument shows that $\lambda(L, M)$ depends only on $\pi(M)$.

(ii) The fact that $\lambda(\cdot, \cdot)$ is a 2-cocycle follows from the definition. Let us show how to rewrite the formula (3.2.4) in the required form for $L=L\left(g_{2}\right)$ and $M=L\left(g_{1}\right)$, where $g_{1}, g_{2} \in U^{0}$ are such that $g_{1} g_{2} \in U^{0}$. Let

$$
g_{i}=\left(\begin{array}{cc}
\alpha_{i} & \beta_{i} \\
\gamma_{i} & \delta_{i}
\end{array}\right), i=1,2
$$

where $\beta_{1}$ and $\beta_{2}$ are invertible. For $i=1,2$ we have isomorphisms in $\mathcal{A} b_{\mathbb{Q}}$

$$
\phi_{i}: A \times \hat{A} \rightarrow L\left(g_{i}\right):(a, \xi) \mapsto\left(a, \xi, \alpha_{i} a+\beta_{i} \xi, \gamma_{i} a+\delta_{i} \xi\right),
$$

Recall that $-\lambda(M, L)$ is the index of the symmetric isogeny $\hat{f}_{0} g_{0}: F_{0} \rightarrow \hat{F}_{0}$, where $F=\operatorname{ker}\left(L\left(g_{2}\right) \times_{A} L\left(g_{1}\right) \stackrel{p_{17}}{\longrightarrow} A \times A\right)$ and $f_{0}$ and $g_{0}$ were defined in Proposition 3.2.9(ii). Here we view $L\left(g_{2}\right) \times{ }_{A} L\left(g_{1}\right)$ as a subvariety in $X_{A} \times X_{A} \times X_{A} \times X_{A}=A \times \hat{A} \times \ldots$ (the 
last product has 8 factors) and denote by $p_{17}$ the corresponding projection to $A \times A$. It is easy to check that there is an isomorphism in $\mathcal{A} b_{\mathbb{Q}}$

$$
\phi: \hat{A} \rightarrow F_{0}: \xi \mapsto\left(0, \xi, \beta_{2} \xi, \delta_{2} \xi\right),\left(\beta_{2} \xi,-\gamma \xi, 0,\left(\gamma_{1} \beta_{2}-\delta_{1} \gamma\right) \xi\right),
$$

where $\gamma=\beta_{1}^{-1} \alpha_{1} \beta_{2}$. Furthermore, we have

$$
f_{0}(\phi(\xi))=\beta_{2} \xi \text { and } g_{0}(\phi(\xi))=\left(\delta_{2}+\gamma\right) \xi
$$

Hence,

$$
\hat{\phi} \hat{f} g \phi=\hat{\beta}_{2}\left(\delta_{2}+\gamma\right)=\hat{\beta}_{2}\left(\delta_{2}+\beta_{1}^{-1} \alpha_{1} \beta_{2}\right)
$$

and we obtain

$$
\lambda\left(g_{1}, g_{2}\right)=i\left(\hat{\beta}_{2}\left(\delta_{2}+\beta_{1}^{-1} \alpha_{1} \beta_{2}\right)\right)=i\left(\left(\delta_{2}+\beta_{1}^{-1} \alpha_{1} \beta_{2}\right) \beta_{2}^{-1}\right)=i\left(\delta_{2} \beta_{2}^{-1}+\beta_{1}^{-1} \alpha_{1}\right)
$$

which is equivalent to the desired formula.

Remark 3.2.15. In the case when $g_{1}, g_{2} \in U\left(X_{A}, \mathbb{Q}\right) \cap \operatorname{End}\left(X_{A}\right)$, our formula for $\lambda\left(g_{1}, g_{2}\right)$ agrees with that of Orlov in [18, Sec. 4], due to the standard formula for the index of a line bundle (see Sec. 1.3).

3.3. Central extensions related to LI-endofunctors. From now on we assume that $\operatorname{char}(k)=0$.

Definition 3.3.1. Let us denote by $\operatorname{KER}(A, A)$ the set of isomorphism classes of objects of $D^{b}(A \times A)$. We equip it with a semiring structure by taking the direct sum $\oplus$ as addition and the convolution $\circ_{A}$ (see Sec. 1.5) as multiplication.

Note that we have a homomorphism of semirings

$$
\operatorname{KER}(A, A)^{o p} \rightarrow \operatorname{Fun}\left(D^{b}(A), D^{b}(A)\right): K \mapsto \Phi_{K},
$$

where $\Phi_{K}$ is the Fourier-Mukai functor given by the kernel $K$ (see Sec. 1.5) and Fun(,$\left.\cdot\right)$ is the set of isomorphism classes of exact functors .

Definition 3.3.2. (i) Let us say that a cohomologically pure kernel $K \in \operatorname{KER}(A, A)$ is an LI-kernel (resp., weak LI-kernel) if there exists a Lagrangian correspondence $(L, \alpha)$ in $\operatorname{Lag}\left(X_{A}\right)$ such that $K$ is $(L, \alpha)$-invariant (resp., $K=\bigoplus_{i=1}^{N} K_{i}$, where $K_{i}$ are $\left(L, \alpha_{i}\right)$ invariant for some Lagrangian correspondences $\left(L, \alpha_{i}\right)$ with common $\left.L\right)$.

(ii) Let $\operatorname{KER}^{L I}(A, A)$ (resp., $\left.\operatorname{KER}^{w L I}(A, A) \subset \operatorname{KER}(A, A) ; \operatorname{resp} ., \operatorname{KER}_{\oplus}^{L I}(A, A) \subset \operatorname{KER}(A, A)\right)$ denote the subset consisting of LI-kernels (resp., weak LI-kernels; resp., finite direct sums of LI-kernels). By Theorem 3.2.14(i) and Corollary 2.4.8 $\operatorname{KER}_{\oplus}^{L I}(A, A)$ is a subsemiring in $\operatorname{KER}(A, A)$, while $\operatorname{KER}^{w L I}(A, A)$ is a submonoid in the mutliplicative monoid $\left(\operatorname{KER}(A, A), \circ_{A}\right)$.

(iii) Consider the following equivalence relation on $\operatorname{KER}^{L I}(A, A)$ (resp., $\left.\operatorname{KER}^{w L I}(A, A)\right)$ : $K \sim_{\mathbf{H}} K^{\prime}$ if there exists a point $x \in X_{A \times A}$ such that $K^{\prime} \simeq T_{x}(K)$ (resp., there exists a direct sum decomposition $K=\bigoplus_{i=1}^{N} K_{i}$ and a collection of points $x_{i} \in X_{A \times A}, i=$ $1, \ldots, N$, such that $K^{\prime} \simeq \bigoplus_{i=1}^{N} T_{x_{i}}\left(K_{i}\right)$.) By Lemmas 3.2.3 and 3.2.4, this is equivalent to the existence of $x^{\prime} \in X_{A}$ and $x^{\prime \prime} \in X_{A}$ such that

$$
K^{\prime} \simeq \mathbb{T}_{x^{\prime}}^{A} \circ_{A} K \simeq K \circ_{A} \mathbb{T}_{x^{\prime \prime}}^{A}
$$


This easily implies that the set of equivalence classes

$$
\overline{\operatorname{KER}}^{L I}(A, A):=\operatorname{KER}^{L I}(A, A) / \sim_{\mathbf{H}} \simeq \operatorname{KER}^{w L I}(A, A) / \sim_{\mathbf{H}}
$$

inherits the monoid structure (with respect to the convolution $\circ_{A}$ ).

We have a central submonoid $Z \subset \overline{\mathrm{KER}}^{L I}(A, A)$ consisting of kernels of the form $\mathcal{O}_{\Delta_{A}}^{\oplus m}[n]$, where $\Delta_{A} \subset A \times A$ is the diagonal. The convolution with an element in $Z$ is a very simple operation:

$$
K \circ_{A} \mathcal{O}_{\Delta}^{\oplus m}[n] \simeq K^{\oplus m}[n] .
$$

We will use the notation $m \cdot K:=K^{\oplus m}$. Note that $Z$ is isomorphic to $\mathbb{N}^{*} \times \mathbb{Z}$, so the localization of $Z$ with respect to the multiplicative set $\mathbb{N}^{*} \subset Z$ gives the group $Z_{\mathbb{Q}^{*}}=\mathbb{Q}^{*} \times \mathbb{Z}$

Let us denote by $\overline{\mathrm{KER}}^{L I}(A, A)_{\mathbb{Q}^{*}}$ the localization of $\overline{\mathrm{KER}}^{L I}(A, A)$ with respect to the central multiplicative set $\mathbb{N}^{*} \subset Z$.

Lemma 3.3.3. The monoid $\overline{\mathrm{KER}}^{L I}(A, A)_{\mathbb{Q}^{*}}$ is a group.

Proof. Let $(L, \alpha)$ be a Lagrangian correspondence from $X_{A}$ to $X_{A}$, such that both projections $L \rightarrow X_{A}$ are surjective. Consider the transposed Lagrangian correspondence $\left(\sigma L, \alpha^{-1}\right)$ and the composed g-Lag-correspondence $(Z, \beta)=\left(L \circ \sigma L, \alpha \circ \alpha^{-1}\right)$. Let $j: Z \rightarrow X_{A} \times X_{A}$ be the natural map, and let $Z_{0}$ be the connected component of zero in $Z$. Since we have the diagonal map $L \rightarrow Z$, we obtain that $j\left(Z_{0}\right)$ is equal to the diagonal $\Delta_{X} \subset X_{A} \times X_{A}$. Therefore, by Theorem 3.2.14 and Proposition 2.4.7(ii), we obtain

$$
S_{L, \alpha} \circ_{A} S_{\sigma L, \alpha^{-1}}=N \cdot S_{\Delta_{X}, \mathcal{O}}[i]=N \cdot \mathcal{O}_{\Delta_{A}}[i]
$$

in $\overline{\mathrm{KER}}^{L I}(A, A)$ with $N \in \mathbb{N}^{*}$ and $i \in \mathbb{Z}$. This shows that $S_{L, \alpha}$ has a right inverse in $\overline{\mathrm{KER}}^{L I}(A, A)_{\mathbb{Q}^{*}}$. Exchanging the roles of $L$ and $\sigma L$ we also obtain the existence of a left inverse. Since every element of $\operatorname{KER}^{L I}(A, A)$ is of the form $n \cdot S_{L, \alpha}$ with $n \in \mathbb{N}^{*}$, we see that it is invertible in $\overline{\mathrm{KER}}^{L I}(A, A)_{\mathbb{Q}^{*}}$.

The first two parts of the following theorem summarize most of the picture discussed above.

Theorem 3.3.4. Assume that $\operatorname{char}(k)=0$.

(i) The semiring $\operatorname{KER}_{\oplus}^{L I}(A, A)$ has as an additive basis the kernels of the form $S_{L(g), \alpha}[n]$, where $L(g)$ is the Lagrangian correspondence from $X_{A}$ to $X_{A}$ associated with $g \in U\left(X_{A}, \mathbb{Q}\right)$ (see Example 3.1.2), $\alpha$ is a line bundle on $L(g)$ such that $(L(g), \alpha)$ is a Lagrangian pair, and $n$ is an integer. The multiplication in $\operatorname{KER}_{\oplus}^{L I}(A, A)$ has form

$$
S_{L\left(g_{2}\right), \alpha_{2}}\left[n_{2}\right] \circ_{A} S_{L\left(g_{1}\right), \alpha_{1}}\left[n_{1}\right]=\bigoplus_{i=1}^{N} S_{L\left(g_{1} g_{2}\right), \beta_{i}}\left[n_{1}+n_{2}+\lambda\left(g_{1}, g_{2}\right)\right]
$$

with $\lambda(\cdot, \cdot)$ as in Theorem 3.2.14 and some line bundles $\beta_{1}, \ldots, \beta_{N}$ on $L\left(g_{1} g_{2}\right)$, such that $\left(L\left(g_{1} g_{2}\right), \beta_{i}\right)$ are Lagrangian pairs.

(ii) For each $g \in U\left(X_{A}, \mathbb{Q}\right)$ let us choose a line bundle $\alpha_{g}$ in such a way that $\left(L(g), \alpha_{g}\right)$ is a Lagrangian pair. Then the class $S(g)$ of the kernel $S_{L(g), \alpha_{g}} \in \operatorname{KER}^{L I}(A, A)$ in 
$\overline{\mathrm{KER}}^{L I}(A, A)$ depends only on $g$. For $g_{1}, g_{2} \in U\left(X_{A}, \mathbb{Q}\right)$ one has the following equality in $\overline{\mathrm{KER}}^{L I}(A, A)$ :

$$
S\left(g_{2}\right) \circ_{A} S\left(g_{1}\right)=N\left(g_{1}, g_{2}\right) \cdot S\left(g_{1} g_{2}\right)\left[\lambda\left(g_{1}, g_{2}\right)\right],
$$

where $N\left(g_{1}, g_{2}\right) \in \mathbb{N}^{*}$. The map $g \mapsto S(g)$ extends to an isomorphism of groups

$$
\widehat{U}\left(X_{A}, \mathbb{Q}\right) \rightarrow{\overline{\mathrm{KER}^{L I}}}^{L I}(A, A)_{\mathbb{Q}^{*}}^{o p},
$$

where $\widehat{U}\left(X_{A}, \mathbb{Q}\right)$ is the central extension of $U\left(X_{A}, \mathbb{Q}\right)$ by $Z_{\mathbb{Q}^{*}}=\mathbb{Q}^{*} \times \mathbb{Z}$ associated with the 2-cocycle $\left(N\left(g_{1}, g_{2}\right), \lambda\left(g_{1}, g_{2}\right)\right)$.

(iii) For $g_{1}, g_{2} \in U\left(X_{A}, \mathbb{Q}\right)$ such that $b\left(g_{1}\right), b\left(g_{2}\right)$ and $b\left(g_{1} g_{2}\right)$ are invertible, one has

$$
N\left(g_{1}, g_{2}\right)=\frac{q\left(L\left(g_{1}\right)\right)^{1 / 2} q\left(L\left(g_{2}\right)\right)^{1 / 2}}{q\left(L\left(g_{1} g_{2}\right)\right)^{1 / 2}} \in \mathbb{N}^{*} .
$$

with $q(L)$ given by (3.1.2).

Proof. The assertion about the additive basis in $\operatorname{KER}_{\oplus}^{L I}(A, A)$ follows from Theorem 2.4.5 and Corollary 2.4.11. The equation (3.3.1) follows from Theorem 3.2.14 together with Corollary 2.4.8.

The fact that the class of $S_{L(g), \alpha_{g}}$ in $\overline{\mathrm{KER}}^{L I}(A, A)$ does not depend on the choice of $\alpha_{g}$ follows immediately from Lemma 2.4.2. Furthermore, together with Corollary 2.4.11 this implies that the map $(g, m, n) \rightarrow S(g)^{\oplus m}[n]$ is a bijection between $U\left(X_{A}, \mathbb{Q}\right) \times \mathbb{N}^{*} \times \mathbb{Z}$ and $\overline{\mathrm{KER}}^{\mathrm{LI}}(A, A)$.

Applying Proposition 2.4.7(ii) to $Z=L\left(g_{1}\right) \circ L\left(g_{2}\right)$ we get

$$
S_{L\left(g_{1}\right) \circ L\left(g_{2}\right), \alpha_{g_{1}} \circ \alpha_{g_{2}}} \simeq\left(\bigoplus_{x \in \Pi^{\prime}} T_{x}\left(S_{L\left(g_{1} g_{2}\right), \alpha_{g_{1} g_{2}}}\right)\right)^{\oplus N_{Z}} .
$$

Recall that here

$$
\left|\Pi^{\prime}\right|=\left|\pi_{0}(j(Z))\right|=d:=\operatorname{deg}\left(Z_{0} \rightarrow j\left(Z_{0}\right)\right)
$$

(see (2.4.12)) and $N_{Z}=\frac{\left|\pi_{0}(Z)\right|^{1 / 2}}{d^{1 / 2}}$. Hence, by Theorem 3.2.14, in $\overline{\mathrm{KER}}^{L I}(A, A)$ we have

$$
S_{L\left(g_{1}\right) \circ L\left(g_{2}\right), \alpha_{g_{1}} \circ \alpha_{g_{2}}}=\left|\pi_{0}(Z)\right|^{1 / 2} \cdot d^{1 / 2} \cdot S\left(g_{1} g_{2}\right)
$$

which implies (3.3.2) with

$$
N\left(g_{1}, g_{2}\right)=\left|\pi_{0}(Z)\right|^{1 / 2} \cdot d^{1 / 2} .
$$

This gives a homomorphism (3.3.3) from the central extension $\widehat{U}\left(X_{A}, \mathbb{Q}\right)$, which is easily seen to be an isomorphism from the above identification of the set $\overline{\mathrm{KER}}^{L I}(A, A)$.

It remains to prove the formula (3.3.4) in the case when $b\left(g_{1}\right), b\left(g_{2}\right)$ and $b\left(g_{1} g_{2}\right)$ are invertible. Note that by Lemma 3.1.7, we have

$$
q(Z)=q\left(L\left(g_{1}\right)\right) \cdot q\left(L\left(g_{2}\right)\right) .
$$

Thus, (3.3.4) can be rewritten as

$$
\pi_{0}(Z) \cdot d=\frac{q(Z)}{q\left(j\left(Z_{0}\right)\right)}=\frac{\operatorname{deg}\left(Z \rightarrow X_{A}\right)}{\operatorname{deg}\left(j\left(Z_{0}\right) \rightarrow X_{A}\right)} .
$$


But this can be checked by comparing the degrees in the commutative diagram of isogenies

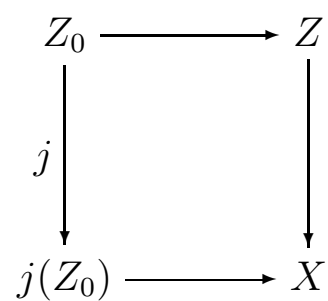

as in the end of proof of Proposition 2.4.7(ii).

Corollary 3.3.5. Let $\overline{\mathrm{KER}}^{L I}(A, A)_{\mathbb{R}^{*}}$ be the push-out of the central extension sequence

$$
1 \rightarrow \mathbb{Q}^{*} \rightarrow{\overline{\mathrm{KER}^{L I}}}^{L I}(A, A)_{\mathbb{Q}^{*}} \rightarrow \overline{\mathrm{KER}}^{L I}(A, A)_{\mathbb{Q}^{*}} / \mathbb{Q}^{*} \rightarrow 1
$$

with respect to the embedding $\mathbb{Q}^{*} \rightarrow \mathbb{R}^{*}$, and let $\widetilde{U}(X, \mathbb{Q})$ be the central extension of $U(X, \mathbb{Q})$ by $\mathbb{Z}$ associated with the 2-cocycle $\lambda(\cdot, \cdot)$. Then the map $g \mapsto S(g) \cdot q(L(g))^{-1 / 2}$ extends to a homomorphism

$$
\widetilde{U}(X, \mathbb{Q}) \rightarrow \overline{\mathrm{KER}}^{L I}(A, A)_{\mathbb{R}^{*}}
$$

The 2-cocycle $N(\cdot, \cdot)$ given by (3.3.4) gives in general a nontrivial cohomology class in $H^{2}\left(U\left(X_{A}, \mathbb{Q}\right), \mathbb{Q}^{*}\right)$ as the following result shows.

Proposition 3.3.6. Let $E$ be an elliptic curve with complex multiplication by $\sqrt{D}$, where $D<-1$ and $|D|$ is square-free. Then the cohomology class in $H^{2}\left(U\left(X_{E}, \mathbb{Q}\right), \mathbb{Q}^{*}\right)$ given by $N(\cdot, \cdot)$ is nontrivial.

Proof. Let $\sqrt{\mathbb{Q}^{*}} \subset \mathbb{R}^{*}$ be the subgroup of $x \in \mathbb{R}^{*}$ such that $x^{2} \in \mathbb{Q}^{*}$. We have a homomorphism

$$
\bar{q}^{1 / 2}: U\left(X_{E}, \mathbb{Q}\right) \rightarrow \sqrt{\mathbb{Q}^{*}} / \mathbb{Q}^{*}: g \mapsto q(L(g))^{1 / 2} \bmod \mathbb{Q}^{*}
$$

that we can view as an element of $H^{1}\left(U\left(X_{E}, \mathbb{Q}\right), \sqrt{\mathbb{Q}^{*}} / \mathbb{Q}^{*}\right)$. Now the class of $N(\cdot, \cdot)$ is equal to $\delta\left(q^{1 / 2}\right)$ where

$$
\delta: H^{1}\left(U\left(X_{E}, \mathbb{Q}\right), \sqrt{\mathbb{Q}^{*}} / \mathbb{Q}^{*}\right) \rightarrow H^{2}\left(U\left(X_{E}, \mathbb{Q}\right), \mathbb{Q}^{*}\right)
$$

is the coboundary map coming from the exact sequence

$$
1 \rightarrow \mathbb{Q}^{*} \rightarrow \sqrt{\mathbb{Q}^{*}} \rightarrow \sqrt{\mathbb{Q}^{*}} / \mathbb{Q}^{*} \rightarrow 1 .
$$

Thus, it is enough to prove that $\bar{q}^{1 / 2}$ cannot be lifted to a homomorphism $U\left(X_{E}, \mathbb{Q}\right) \rightarrow$ $\sqrt{\mathbb{Q}^{*}}$. We are going to prove that it cannot even be lifted to a homomorphism $U\left(X_{E}, \mathbb{Q}\right) \rightarrow$ $\sqrt{\mathbb{Q}^{*}} / \pm 1$. Indeed, using the isomorphism $\sqrt{\mathbb{Q}^{*}} / \pm 1 \rightarrow \mathbb{Q}^{*}: x \mapsto x^{2}$ we see that this is equivalent to asserting that the homomorphism

$$
\bar{q}: S p\left(X_{E}, \mathbb{Q}\right) \rightarrow \mathbb{Q}^{*} /\left(\mathbb{Q}^{*}\right)^{2}: g \mapsto q(L(g)) \bmod \left(\mathbb{Q}^{*}\right)^{2}
$$

cannot be lifted to a homomorphism to $\mathbb{Q}^{*}$. 
Note that the group $U\left(X_{E}, \mathbb{Q}\right)$ in our case is isomorphic to the unitary group $U=$ $U_{2}(K, f)$, where $K=\mathbb{Q}(\sqrt{D})$ and $f$ is a skew-Hermitian form of index 1 (we use the terminology of [6]). Let us consider the natural embedding

$$
\iota: K^{*} / \mathbb{Q}^{*} \rightarrow K^{*}: a \mapsto \frac{\bar{a}}{a} .
$$

It is known that the image of the determinant map det :U $\rightarrow K^{*}$ is contained in $\iota\left(K^{*} / \mathbb{Q}^{*}\right)$ (see [5, Thm. 3]). Hence, we have a unique homomorphism $\phi: U \rightarrow K^{*} / \mathbb{Q}^{*}$ such that $\operatorname{det}(g)=\iota(\phi(g))$. We claim that

$$
\bar{q}(g)=\overline{\mathrm{Nm}}(\phi(g)),
$$

where $\overline{\mathrm{Nm}}: K^{*} / \mathbb{Q}^{*} \rightarrow \mathbb{Q}^{*} /\left(\mathbb{Q}^{*}\right)^{2}$ is induced by the norm homomorphism $\mathrm{Nm}: K^{*} \rightarrow \mathbb{Q}^{*}$. Indeed, it is known that in our case the special unitary group $S U \subset U$ coincides with the normal subgroup $T \subset U$ generated by unitary transvections (see $[6, \S 5]$ ), and the latter subgroup coincides with $\mathrm{SL}_{2}(\mathbb{Q}) \subset U$. It is easy to see that for $g \in \mathrm{SL}_{2}(\mathbb{Q})$ the degree of the projection $p_{12}: L(g) \rightarrow E^{2}$ is a square. Thus, $\bar{q}$ is trivial on $S U$ and we should have

$$
\bar{q}(g)=\chi(\phi(g))
$$

for some homomorphism $\chi: K^{*} / \mathbb{Q}^{*} \rightarrow \mathbb{Q}^{*} /\left(\mathbb{Q}^{*}\right)^{2}$. It remains to check that our statement holds for diagonal matrices

$$
g_{a}=\left(\begin{array}{cc}
a^{-1} & 0 \\
0 & \bar{a}
\end{array}\right)
$$

where $a \in K^{*}\left(\right.$ since $\left.\phi\left(g_{a}\right)=a\right)$. In other words, we claim that in this case

$$
\bar{q}\left(L\left(g_{a}\right)\right) \equiv \operatorname{Nm}(a) \bmod \left(\mathbb{Q}^{*}\right)^{2} .
$$

It is enough to check this in the case when $a \in \operatorname{End}(E) \subset K$. Then the Lagrangian correspondence $L\left(g_{a}\right)$ can be described as

$$
L\left(g_{a}\right)=\{(a x, y, x, \bar{a} y) \mid x, y \in E\} \subset E^{2} \times E^{2},
$$

so $q\left(L\left(g_{a}\right)\right)=\operatorname{deg}(a)=\operatorname{Nm}(a)$.

Finally, suppose we have a homomorphism $\rho: U \rightarrow \mathbb{Q}^{*}$ such that $\overline{\mathrm{Nm}}(\phi(g))=$ $\rho(g) \bmod \left(\mathbb{Q}^{*}\right)^{2}$. Let us specialize this equality to $g=g_{\sqrt{D}}$. Then we should have

$$
\rho\left(g_{\sqrt{D}}\right) \equiv-D \bmod \left(\mathbb{Q}^{*}\right)^{2} .
$$

But $\rho$ factors through $U /[U, U]$ and $g_{\sqrt{D}}$ projects to an element of finite order in $U /[U, U]$. Therefore, $\rho\left(g_{\sqrt{D}}\right)$ should be an element of finite order in $\mathbb{Q}^{*}$, i.e., $\rho\left(g_{\sqrt{D}}\right)= \pm 1$ which is a contradiction.

\section{REFERENCES}

[1] A. Borel, Linear algebraic groups, 2nd edition, Springer-Verlag, New York, 1991.

[2] T. Bridgeland, Derived categories of coherent sheaves, ICM, Vol. II, 563-582, Eur. Math. Soc., Zürich, 2006.

[3] T. Bridgeland, Stability conditions on K3 surfaces, Duke Math. J. 141 (2008), no. 2, 241-291.

[4] P. Deligne, Théorie de Hodge, III, Publ. Math. IHES 44 (1974), 5-78.

[5] J. Dieudonné, On the structure of unitary groups. II, Amer. J. Math. 75 (1953). 665-678. 
[6] J. Dieudonné, La géometrie des groupes classiques, Troisième édition, Springer-Verlag, Berlin-New York, 1971.

[7] A. D. Elagin, Descent theory for derived categories, Russian Math. Surveys 64 (2009), no. 4, 748-749.

[8] G. Laumon, Transformation de Fourier generalisée, preprint alg-geom/9603004.

[9] M. Inaba, Toward a definition of moduli of complexes of coherent sheaves on a projective scheme, J. Math. Kyoto Univ. 42 (2002), no. 2, 317-329.

[10] M. Kontsevich, Y. Soibelman, Notes on A-infinity algebras, A-infinity categories and noncommutative geometry, in Homological mirror symmetry, 153-219, Lecture Notes in Phys. 757, Springer, Berlin, 2009.

[11] Yu. I. Manin, Real Multiplication and noncommutative geometry (ein Alterstraum), in The legacy of Niels Henrik Abel, 685-727, Springer, Berlin, 2004.

[12] L. Moret-Bailly, Pinceaux de variétés abéliennes, Astérisque 129 (1985).

[13] S. Mukai, Duality between $D(X)$ and $D(\hat{X})$ with its application to Picard sheaves, Nagoya Math. J. 81 (1981), 153-175.

[14] S. Mukai, Semi-homogeneous vector bundles on an abelian variety, J. Math. Kyoto Univ. 18 (1978), 239-272.

[15] S. Mukai, Abelian variety and spin representation (in Japanese), in Hodge theory and algebraic geometry (Sapporo, 1994), English translation: Univ. of Warwick preprint, 1998.

[16] D. Mumford, Abelian varieties, Tata Institute of Fundamental Research Studies in Math. 5. Oxford University Press, Oxford, 1970.

[17] D. Orlov, Equivalences of derived categories and K3 surfaces, in Algebraic geometry, 7, J. Math. Sci. (New York) 84 (1997), no. 5, 1361-1381.

[18] D. Orlov, Derived categories of coherent sheaves on abelian varieties and equivalences between them, Izv. Math. 66 (2002), no. 3, 569-594.

[19] A. Polishchuk, Biextensions, Weil representation on derived categories and theta-functions. Harvard Ph.D. thesis, 1996.

[20] A. Polishchuk, Symplectic biextensions and a generalization of the Fourier-Mukai transform, Math. Research Letters 3 (1996), 813-828.

[21] A. Polishchuk, Analogue of Weil representation for abelian schemes, J. reine angew. Math. 543 (2002), 1-37.

[22] A. Polishchuk, Abelian Varieties, Theta Functions and the Fourier Transform, Cambridge University Press, 2003.

[23] A. Polishchuk, Kernel algebras and generalized Fourier-Mukai transforms, J. Noncomm. Geom. 5 (2011), 153-251.

[24] A. Polishchuk, A. Schwarz, Categories of holomorphic vector bundles on noncommutative two-tori, Comm. Math. Phys. 236 (2003), 135-159.

[25] B. Toën, The homotopy theory of dg-categories and derived Morita theory, Invent. Math. 167 (2007), $615-667$.

[26] H. Umemura, On a certain type of vector bundles over an abelian variety, Nagoya Math. J. 64 (1976), $31-45$.

[27] A. Weil, Sur certains groupes d'opérateurs unitaires, Acta Math. 111 (1964), 143-211.

Department of Mathematics, University of Oregon, Eugene, OR 97405

E-mail address: apolish@uoregon.edu 